\title{
A BIJECTION BETWEEN LITTLEWOOD-RICHARDSON TABLEAUX AND RIGGED CONFIGURATIONS
}

\author{
ANATOL N. KIRILLOV, ANNE SCHILLING*, AND MARK SHIMOZONO ${ }^{\dagger}$
}

\begin{abstract}
We define a bijection from Littlewood-Richardson tableaux to rigged configurations and show that it preserves the appropriate statistics. This proves in particular a quasi-particle expression for the generalized Kostka polynomials $K_{\lambda R}(q)$ labeled by a partition $\lambda$ and a sequence of rectangles $R$. The generalized Kostka polynomials are $q$-analogues of multiplicities of the irreducible $G L(n, \mathbb{C})$-module $V^{\lambda}$ of highest weight $\lambda$ in the tensor product $V^{R_{1}} \otimes \cdots \otimes V^{R_{L}}$.
\end{abstract}

\section{INTRODUCTION}

In their study of the $X X X$ model using Bethe Ansatz techniques, Kirillov and Reshetikhin [12] obtained an expression for the Kostka polynomials as the generating function of rigged configurations. Rigged configurations index the solutions of the Bethe Ansatz equations; they are sequences of partitions obeying certain conditions together with quantum numbers or riggings labeling the parts of the partitions. In ref. [12] a statistic-preserving bijection between column-strict Young tableaux and rigged configurations was given which connects the generating function of rigged configurations to the charge representation of the Kostka polynomials as obtained by Lascoux and Schützenberger 16.

In fact, the representation of the Kostka polynomials in terms of rigged configurations is exactly in quasi-particle form. In recent years, much research has been devoted to the study of quasi-particle representations of characters of conformal field theories and configuration sums of exactly solvable lattice models (see for example [1, 2, 3, 4, 5, 0, 8, 20, 26, 27] and references therein). These quasi-particle representations are physically interesting [9, 10] because they reflect the particle structure of the underlying model.

Recently certain generalizations of the Kostka polynomials were introduced and studied [13, 20, 22, 23, 24, 25]. These generalized Kostka polynomials $K_{\lambda R}(q)$ are labeled by a partition $\lambda=\left(\lambda_{1}, \lambda_{2}, \ldots\right)$ and a sequence

Date: December 1998.

1991 Mathematics Subject Classification. Primary 05A19, 05A15.

Key words and phrases. Littlewood-Richardson tableaux, rigged configurations, generalized Kostka polynomials.

* Supported by the "Stichting Fundamenteel Onderzoek der Materie".

† Partially supported by NSF grant DMS-9800941. 
of rectangles $R=\left(R_{1}, \ldots, R_{L}\right)$, that is, each $R_{i}=\left(\eta_{i}^{\mu_{i}}\right)$ is a partition of rectangular shape. They are $q$-analogues of multiplicities of the irreducible $G L(n, \mathbb{C})$-module $V^{\lambda}$ of highest weight $\lambda$ in the tensor product $V^{R_{1}} \otimes \cdots \otimes V^{R_{L}}$. This multiplicity is equal to the cardinality of the set of Littlewood-Richardson tableaux [6]. When all $R_{i}$ are single rows (in which case $\left.R_{i}=\left(\eta_{i}\right)\right)$, the generalized Kostka polynomial $K_{\lambda R}(q)$ reduces to the Kostka polynomial $K_{\lambda \eta}(q)$. Conjecturally, the generalized Kostka polynomials coincide with special cases of the spin generating functions over ribbon tableaux of Lascoux, Leclerc and Thibon [15].

In refs. [20, 22] the generalized Kostka polynomials were expressed as the generating function of Littlewood-Richardson tableaux with a generalized charge statistic, extending the result for the Kostka polynomials of Lascoux and Schützenberger [16]. A representation of the generalized Kostka polynomials in terms of rigged configurations was conjectured in [13, 20].

In this paper it is shown that the algorithm described in [11] gives a statistic-preserving bijection between Littlewood-Richardson tableaux and rigged configurations. This bijection extends the bijection between columnstrict Young tableaux and rigged configurations [12], and in particular provides a proof of the quasi-particle representation of the generalized Kostka polynomials as conjectured in [13, 20].

This paper is organized as follows. In Section the set of LittlewoodRichardson (LR) tableaux and the set of rigged configurations corresponding to a sequence of rectangles are defined. The charge expression of the generalized Kostka polynomials is recalled and the quasi-particle representation is stated. In Section 3 several operations on rectangles and their analogues on LR tableaux and rigged configurations are discussed. These operations are crucial for the definition of the bijection between LR tableaux and rigged configurations as given in Definition-Proposition 4.1. The definition of the bijection is based on the operations of splitting off the last column of the last rectangle in $R$ and, if the last rectangle is a single column, removing one box from it. The algorithm of ref. [11] for the bijection, which is computationally simpler but less suitable for the proofs, is stated in Section 4.2. The Evacuation Theorem 5.6 is proven in Section 5. It states that under the bijection the evacuation of LR tableaux corresponds to the complementation of quantum numbers on rigged configurations. In Section 6 another recurrence for $\bar{\phi}_{R}$ is given based on operations involving rows instead of columns as used in Definition-Proposition 4.1. This formulation of $\bar{\phi}_{R}$ is in a sense transpose to the description of $\bar{\phi}_{R^{t}}$ using the column operations where $R^{t}=\left(R_{1}^{t}, \ldots, R_{L}^{t}\right)$ and $R_{i}^{t}$ is the transpose of $R_{i}$. It is used in Section 7 to prove the Transpose Theorem 7.1. Like the LR coefficients, the generalized Kostka polynomials have a transpose symmetry which has been explained combinatorially by a transpose bijection on LR tableaux [20, 23] and by a transpose bijection on rigged configurations [13. The Transpose Theorem shows that the bijection from LR tableaux to rigged configurations intertwines these two transpose bijections. In refs. [20, 23] families of 
statistic-preserving embeddings between sets of LR tableaux were defined. The Embedding Theorem 8.3 of Section 8 shows that these embeddings on

LR tableaux correspond to an inclusion on rigged configurations under the bijection between LR tableaux and rigged configurations. The proof of the Embedding Theorem relies on the Evacuation Theorem. In Section 9 it is finally shown that the bijection is statistic preserving. The proof uses the Transpose Theorem 7.1 and the Embedding Theorem 8.3 to reduce to the case that all rectangles in $R$ are single boxes. In the single box case the property that the bijection is statistic preserving can be checked explicitly. Some technical proofs are relegated to Appendices A and B.

\section{Charge and quasi-Particle Representation of the GENERALIZED KOSTKA POLYNOMIALS}

In this section we define the set of Littlewood-Richardson tableaux and the set of rigged configurations, and recall the charge representation of the generalized Kostka polynomials (2.3). The quasi-particle representation of the generalized Kostka polynomials, to be proven in this paper, is stated in Theorem 2.10.

2.1. Littlewood-Richardson tableaux. Given a partition $\lambda$ and a sequence of partitions $R=\left(R_{1}, \ldots, R_{L}\right)$, define the tensor product multiplicity

$$
c_{\lambda}^{R}=\operatorname{dim} \operatorname{Hom}_{G L(n)}\left(V^{\lambda}, V^{R_{1}} \otimes \cdots \otimes V^{R_{L}}\right)
$$

where $V^{\lambda}$ is the irreducible $G L(n, \mathbb{C})$-module of highest weight $\lambda$. There are many well-known equivalent formulations of the celebrated LittlewoodRichardson rule, which give the multiplicity $c_{\lambda}^{R}$ as the cardinality of a certain set of tableaux (see [6]). We refer to sets of tableaux that have this cardinality as LR tableaux. Here we recall various well-known notions of LR tableaux, which count the multiplicity $c_{\lambda}^{R}$ when $R$ is a sequence of rectangles. One of these is particularly well-suited for use with the bijection to rigged configurations.

First a few tableau definitions are necessary. The English convention is used here for partition diagrams and tableaux. For a partition $\lambda$ denote by $\operatorname{ST}(\lambda)$ the set of standard tableaux of shape $\lambda$. Given a possibly skew column-strict tableau $T$ in an alphabet $A$ and $B$ a subinterval of $A$, denote by $\left.T\right|_{B}$ the restriction of $T$ to $B$, which is by definition the skew column-strict tableau given by erasing from $T$ the letters that are not in $B$. Define the row-reading word of $T$ to be the concatenation $\operatorname{word}(T)=\ldots v^{2} v^{1}$, where $v^{r}$ is the word given by the $r$-th row of $T$ read from left to right. Knuth 14 defined an equivalence relation on words denoted $v \cong_{K} w$. Given a word $w$ there is a unique column-strict tableau $P(w)$ such that word $(P(w)) \cong_{K} w$; this is the Schensted $P$-tableau of the word $w$. Write $P(T):=P(\operatorname{word}(T))$ for the skew column strict tableau $T$. 
Let $R=\left(R_{1}, \ldots, R_{L}\right)$ be a sequence of partitions. For $1 \leq j \leq L$ let $B_{j}$ be an interval of integers such that if $i<j, x \in B_{i}$ and $y \in B_{j}$, then $x<y$. Set $B=\bigcup_{j=1}^{L} B_{j}$. Let $Z_{j}$ be any column-strict tableau of shape $R_{j}$ in the alphabet $B_{j}$ for $1 \leq j \leq L$. Define the set $\operatorname{SLR}(\lambda ; Z)$ to be the set of columnstrict tableaux $T$ of shape $\lambda$ in the alphabet $B$ such that $P\left(\left.T\right|_{B_{j}}\right)=Z_{j}$ for all $j$. It is well-known that $|\operatorname{SLR}(\lambda ; Z)|=c_{\lambda}^{R}$.

Example 2.1. Suppose $R_{j}$ has $\mu_{j}$ parts for all $j$. Define $B_{j}=\left[\mu_{1}+\cdots+\right.$ $\left.\mu_{j-1}+1, \mu_{1}+\cdots+\mu_{j-1}+\mu_{j}\right]$. Let $Z_{j}$ be the column-strict tableau of shape $R_{j}$ whose $r$-th row is filled with copies of the $r$-th largest letter of $B_{j}$, namely, $\mu_{1}+\cdots+\mu_{j-1}+r$. Then the set $\operatorname{SLR}(\lambda ; Z)$ is equal to the set of LR tableaux $\operatorname{LRT}(\lambda ; R)$, which was defined in 13, 20] for the case that each $R_{j}$ is a rectangle.

Example 2.2. Let $\left|R_{j}\right|=N_{j}$ and $N=N_{1}+\cdots+N_{L}$. Define the successive subintervals of $[1, N]$ given by $B_{j}=\left[N_{1}+\cdots+N_{j-1}+1, N_{1}+\cdots+N_{j-1}+N_{j}\right]$ for $1 \leq j \leq L$. Let $Z_{j}$ be any standard tableau of shape $R_{j}$ in the alphabet $B_{j}$. Then the set $\operatorname{SLR}(\lambda ; Z)$ is given by the set of standard tableaux of shape $\lambda$ that is compatible with a certain labeling of the cells of the partitions $R_{j}$ (see [19, 28]).

Remark 2.3. In the situation of standard tableaux as given in Example 2.2 , if partitions $R_{j}$ are rectangles, there is a much simpler characterization of $\operatorname{SLR}(\lambda ; Z)$. Namely, the standard tableau $S$ of shape $\lambda$ is in $\operatorname{SLR}(\lambda ; Z)$ if and only if, for every index $j$ and every pair of letters $x$ and $y$ in $B_{j}$, if $y$ is immediately south (resp. west) of $x$ in $Z_{j}$, then in $S, y$ is in a row (resp. column) strictly south (resp. west) of that of $x$.

Example 2.4. In the characterization of $\operatorname{SLR}(\lambda ; Z)$ given in Remark 2.3, it is important that each $R_{j}$ be a rectangle. Take $\lambda=(2,2)$ and $R=$ $((1),(2,1))$. Then for any choice of $Z_{1}$ and $Z_{2},|\operatorname{SLR}(\lambda ; Z)|=1$, but there are no tableaux satisfying the criterion in Remark 2.3.

Let us fix two canonical choices for the $Z_{j}$. The columnwise standard tableau $\mathrm{CW}(\nu)$ of the partition shape $\nu$ is given by placing the numbers $\nu_{1}^{t}+\cdots+\nu_{c-1}^{t}+1$ through $\nu_{1}^{t}+\cdots+\nu_{c}^{t}$ from top to bottom in the $c$-th column for all $c$. Let $T+x$ denote the tableau obtained by adding $x$ to every entry of the tableau $T$. Given a sequence of rectangles $R$, define the sequence of tableaux $\mathrm{ZC}_{j}(1 \leq j \leq L)$ by $\mathrm{ZC}_{j}=\mathrm{CW}\left(R_{j}\right)+\left|R_{j-1}\right|+\cdots+\left|R_{2}\right|+\left|R_{1}\right|$. Similarly, one can define the rowwise standard tableau $\operatorname{RW}(\nu)$ of shape $\nu$ given by placing the numbers $\nu_{1}+\cdots+\nu_{r-1}+1$ through $\nu_{1}+\cdots+\nu_{r}$ from left to right in the $r$-th row, for all $r$. Define $\mathrm{ZR}_{j}=\mathrm{RW}\left(R_{j}\right)+\left|R_{j-1}\right|+\cdots+$ $\left|R_{2}\right|+\left|R_{1}\right|$ for $1 \leq j \leq L$.

Definition 2.5. Set

$$
\begin{aligned}
& \operatorname{CLR}(\lambda ; R):=\operatorname{SLR}\left(\lambda ;\left(\mathrm{ZC}_{1}, \ldots, \mathrm{ZC}_{L}\right)\right), \\
& \operatorname{RLR}(\lambda ; R):=\operatorname{SLR}\left(\lambda ;\left(\mathrm{ZR}_{1}, \ldots, \mathrm{ZR}_{L}\right)\right) .
\end{aligned}
$$


The set $\operatorname{CLR}(\lambda ; R)$ will be used for the bijection with rigged configurations.

Observe that for any choice of $Z_{j}$ and $Z_{j}^{\prime}$ (with $R_{j}$ a rectangle for all $j)$, a bijection $\operatorname{SLR}(\lambda ; Z) \rightarrow \operatorname{SLR}\left(\lambda ; Z^{\prime}\right)$ is given by relabeling. Namely, let $S \in \operatorname{SLR}(\lambda ; Z)$. Then for each $j$ and each cell $s$ in $R_{j}$, replace the letter $Z_{j}(s)$ in $S$ by $Z_{j}^{\prime}(s)$. In particular there is a bijection

$$
\gamma_{R}: \operatorname{CLR}(\lambda ; R) \rightarrow \operatorname{RLR}(\lambda ; R)
$$

Note that the ordinary transposition of standard tableaux $\operatorname{tr}: \operatorname{ST}(\lambda) \rightarrow$ $\mathrm{ST}\left(\lambda^{t}\right)$ restricts to a bijection

$$
\operatorname{tr}: \operatorname{CLR}(\lambda ; R) \rightarrow \operatorname{RLR}\left(\lambda^{t} ; R^{t}\right)
$$

Here $\lambda^{t}$ stands for the transpose of the partition $\lambda$ and $R^{t}=\left(R_{1}^{t}, \ldots, R_{L}^{t}\right)$ is the sequence of rectangles obtained by transposing each rectangle of $R$.

Remark 2.6. A bijection $\beta_{R}: \operatorname{LRT}(\lambda ; R) \rightarrow \operatorname{CLR}(\lambda ; R)$ is given by a trivial relabeling. Recall the alphabets $B_{1}$ through $B_{L}$ from Example 2.1. Let $T \in \operatorname{LRT}(\lambda ; R)$. Then the tableau $\beta_{R}(T)$ is obtained from $T$ by replacing the occurrences of the $r$-th largest letter of the subalphabet $B_{j}$, from left to right, by the numbers in the $r$-th row of $\mathrm{ZC}_{j}$. Alternatively, $\beta_{R}=\gamma_{R}^{-1} \circ$ std where the bijection std $: \operatorname{LRT}(\lambda ; R) \rightarrow \mathrm{ST}(\lambda)$ is Schensted's standardization map. Also $\beta_{R}=\operatorname{tr} \circ$ std $\circ \operatorname{tr}_{\mathrm{LR}}^{\prime}$, where $\operatorname{tr}_{\mathrm{LR}}^{\prime}$ is the LR-transpose map $\operatorname{LRT}(\lambda ; R) \rightarrow$ $\operatorname{LRT}\left(\lambda^{t} ; R^{t}\right)$ defined in [13].

Definition 2.7. The bijection $\operatorname{tr}_{\mathrm{LR}}: \operatorname{CLR}(\lambda ; R) \rightarrow \operatorname{CLR}\left(\lambda^{t} ; R^{t}\right)$ is given by $\operatorname{tr}_{\mathrm{LR}}:=\operatorname{tr} \circ \gamma_{R}=\gamma_{R^{t}} \circ \operatorname{tr}$ where $\gamma_{R}$ and $\operatorname{tr}$ are defined in (2.1) and (2.2) respectively.

Example 2.8. Let $R=((3,3),(2,2,2,2),(1,1,1))$ as in [13, Example 10]. We give the set $\operatorname{CLR}(\lambda ; R) . B_{1}=[1,6], B_{2}=[7,14], B_{3}=[15,17]$, and

$$
Z_{1}=\begin{array}{rrrr}
1 & 3 & 5 \\
2 & 4 & 6
\end{array} \quad Z_{2}=\begin{array}{rrr}
7 & 11 \\
8 & 12 \\
9 & 13 \\
10 & 14
\end{array} \quad Z_{3}=\begin{array}{r}
15 \\
17
\end{array}
$$


For $\lambda=(5,4,3,2,2,1)$, the set $\operatorname{CLR}(\lambda ; R)$ is given below, listed in order as the images of the set $\operatorname{LRT}(\lambda ; R)$ in [13, Example 11] under the bijection $\beta_{R}$.

$\begin{array}{cccccccccc}1 & 3 & 5 & 7 & 11 & 1 & 3 & 5 & 7 & 11 \\ 2 & 4 & 6 & 12 & & 2 & 4 & 6 & 15 & \\ 8 & 13 & 15 & & & 8 & 12 & 16 & & \\ 9 & 14 & & & & 9 & 13 & & & \\ 10 & 16 & & & & 10 & 14 & & & \\ 17 & & & & & 17 & & & & \end{array}$

$\begin{array}{cccccccccc}1 & 3 & 5 & 11 & 15 & 1 & 3 & 5 & 11 & 15 \\ 2 & 4 & 6 & 12 & & 2 & 4 & 6 & 16 & \\ 7 & 13 & 16 & & & 7 & 12 & 17 & & \\ 8 & 14 & & & & 8 & 13 & & & \\ 9 & 17 & & & & 9 & 14 & & & \\ 10 & & & & & 10 & & & & \end{array}$

Example 2.9. When $R=\left((1)^{N}\right)$ and $\lambda$ is a partition of $N, \operatorname{CLR}(\lambda ; R)=$ $\operatorname{ST}(\lambda)$. When $R=\left(\left(\eta_{1}\right), \ldots,\left(\eta_{L}\right)\right)$, $\operatorname{LRT}(\lambda ; R)=\operatorname{CST}(\lambda ; \eta)$, the set of column-strict tableaux of shape $\lambda$ and content $\eta$, and $\beta_{R}$ is Schensted's standardization map.

It was shown in refs. 20, 22, that the set $\operatorname{LRT}(R)=\cup_{\lambda} \operatorname{LRT}(\lambda ; R)$ has the structure of a graded poset with covering relation given by the $R$-cocyclage and grading function given by the generalized charge, denoted $c_{R}$. The bijection $\beta_{R}$ also induces a ranked poset structure on $\operatorname{CLR}(R)=\cup_{\lambda} \operatorname{CLR}(\lambda ; R)$; by abuse of notation we denote its grading function also by $c_{R}$. The generalized Kostka polynomial is the generating function of LR tableaux with charge statistic 20, 22,

$$
K_{\lambda R}(q)=\sum_{T \in \operatorname{CLR}(\lambda ; R)} q^{c_{R}(T)}
$$

This extends the charge representation of the Kostka polynomials $K_{\lambda \eta}(q)$ of Lascoux and Schützenberger [16, 17]. The generalized Kostka polynomials $K_{\lambda R}(q)$ specialize to $K_{\lambda \eta}(q)$ when $R=\left(\left(\eta_{1}\right), \ldots,\left(\eta_{L}\right)\right)$ is a sequence of single rows.

2.2. Rigged configurations. Let $R=\left(R_{1}, \ldots, R_{L}\right)$ be a sequence of rectangular partitions such that $R_{j}$ has $\mu_{j}$ rows and $\eta_{j}$ columns for $1 \leq j \leq L$; this convention differs from 13]. Denote by $|\lambda|$ the size of the partition $\lambda$ and set $|R|=\left|R_{1}\right|+\cdots+\left|R_{L}\right|$. For $|\lambda|=|R|$ a $\left(\lambda^{t} ; R^{t}\right)$-configuration is a sequence of partitions $\nu=\left(\nu^{(1)}, \nu^{(2)}, \ldots\right)$ with the size constraints

$$
\left|\nu^{(k)}\right|=\sum_{j>k} \lambda_{j}^{t}-\sum_{a=1}^{L} \mu_{a} \max \left(\eta_{a}-k, 0\right) .
$$


Define $m_{n}(\rho)$ as the number of parts of the partition $\rho$ equal to $n$ and $Q_{n}(\rho)=\rho_{1}^{t}+\rho_{2}^{t}+\cdots+\rho_{n}^{t}$, the size of the first $n$ columns of $\rho$. Let $\xi^{(k)}(R)$ be the partition whose parts are the heights of the rectangles in $R$ of width $k$. The vacancy numbers for the $\left(\lambda^{t} ; R^{t}\right)$-configuration $\nu$ are the numbers (indexed by $k \geq 1$ and $n \geq 0$ ) defined by

$$
P_{n}^{(k)}(\nu)=Q_{n}\left(\nu^{(k-1)}\right)-2 Q_{n}\left(\nu^{(k)}\right)+Q_{n}\left(\nu^{(k+1)}\right)+Q_{n}\left(\xi^{(k)}(R)\right)
$$

where $\nu^{(0)}$ is the empty partition by convention. In particular $P_{0}^{(k)}(\nu)=0$ for all $k \geq 1$. The $\left(\lambda^{t} ; R^{t}\right)$-configuration $\nu$ is admissible if $P_{n}^{(k)}(\nu) \geq 0$ for all $k, n \geq 1$, and the set of admissible $\left(\lambda^{t} ; R^{t}\right)$-configurations is denoted by $\mathrm{C}\left(\lambda^{t} ; R^{t}\right)$. Set

$$
c c(\nu)=\sum_{k, n \geq 1} \alpha_{n}^{(k)}\left(\alpha_{n}^{(k)}-\alpha_{n}^{(k+1)}\right)
$$

where $\alpha_{n}^{(k)}$ is the size of the $n$-th column in $\nu^{(k)}$. Finally, define the $q$ binomial as

$$
\left[\begin{array}{c}
m+n \\
m
\end{array}\right]=\frac{(q)_{m+n}}{(q)_{m}(q)_{n}}
$$

for $m, n \in \mathbb{Z}_{\geq 0}$ and zero otherwise where $(q)_{m}=(1-q)\left(1-q^{2}\right) \cdots\left(1-q^{m}\right)$.

With this notation we can state the following quasi-particle expression of the generalized Kostka polynomials conjectured in [13, 20], stemming from the analogous expression of Kirillov and Reshetikhin [12] for the Kostka polynomial.

Theorem 2.10 (Quasi-particle representation). For $R$ a sequence of rectangles and $\lambda$ a partition such that $|\lambda|=|R|$

$$
K_{\lambda R}(q)=\sum_{\nu \in \mathrm{C}\left(\lambda^{t} ; R^{t}\right)} q^{c c(\nu)} \prod_{k, n \geq 1}\left[\begin{array}{c}
P_{n}^{(k)}(\nu)+m_{n}\left(\nu^{(k)}\right) \\
m_{n}\left(\nu^{(k)}\right)
\end{array}\right] .
$$

Expression (2.6) can be reformulated as the generating function over rigged configurations. To this end we need to define certain labelings of the rows of the partitions in a configuration. For this purpose one should view a partition as a multiset of positive integers. A rigged partition is by definition a finite multiset of pairs $(n, x)$ where $n$ is a positive integer and $x$ is a nonnegative integer. The pairs $(n, x)$ are referred to as strings; $n$ is referred to as the length or size of the string and $x$ as the label or quantum number of the string. A rigged partition is said to be a rigging of the partition $\rho$ if the multiset consisting of the sizes of the strings, is the partition $\rho$. So a rigging of $\rho$ is a labeling of the parts of $\rho$ by nonnegative integers, where one identifies labelings that differ only by permuting labels among equal-sized parts of $\rho$.

A rigging $J$ of the $\left(\lambda^{t} ; R^{t}\right)$-configuration $\nu$ is a sequence of riggings of the partitions $\nu^{(k)}$ such that every label $x$ of a part of $\nu^{(k)}$ of size $n$, satisfies the 
inequalities

$$
0 \leq x \leq P_{n}^{(k)}(\nu)
$$

The pair $(\nu, J)$ is called a rigged configuration. The set of riggings of admissible $\left(\lambda^{t} ; R^{t}\right)$-configurations is denoted by $\operatorname{RC}\left(\lambda^{t} ; R^{t}\right)$. Let $(\nu, J)^{(k)}$ be the $k$-th rigged partition of $(\nu, J)$. A string $(n, x) \in(\nu, J)^{(k)}$ is said to be singular if $x=P_{n}^{(k)}(\nu)$, that is, its label takes on the maximum value.

Remark 2.11. Observe that the definition of the set $\mathrm{RC}\left(\lambda^{t} ; R^{t}\right)$ is completely insensitive to the order of the rectangles in the sequence $R$. However the notation involving the sequence $R$ is useful when discussing the bijection $\bar{\phi}_{R}: \operatorname{CLR}(\lambda ; R) \rightarrow \operatorname{RC}\left(\lambda^{t} ; R^{t}\right)$, since the ordering on $R$ is essential in the definition of $\operatorname{CLR}(\lambda ; R)$.

The set of rigged configurations is endowed with a natural statistic $c c$ 13, (3.2)] defined by

$$
c c(\nu, J)=c c(\nu)+\sum_{k, n \geq 1}\left|J_{n}^{(k)}\right|
$$

for $(\nu, J) \in \mathrm{RC}\left(\lambda^{t} ; R^{t}\right)$. Here $J_{n}^{(k)}$ denotes the partition inside the rectangle of height $m_{n}\left(\nu^{(k)}\right)$ and width $P_{n}^{(k)}(\nu)$ given by the labels of the parts of $\nu^{(k)}$ of size $n$. Since the $q$-binomial $\left[\begin{array}{c}P+m \\ m\end{array}\right]$ is the generating function of partitions with at most $m$ parts each not exceeding $P$, Theorem 2.10 is equivalent to the following theorem.

Theorem 2.12 (Rigged configuration representation). For $R$ a sequence of rectangles and $\lambda$ a partition such that $|\lambda|=|R|$

$$
K_{\lambda R}(q)=\sum_{(\nu, J) \in \operatorname{RC}\left(\lambda^{t} ; R^{t}\right)} q^{c c(\nu, J)} .
$$

The proof of this theorem follows from the bijection $\bar{\phi}_{R}: \operatorname{CLR}(\lambda ; R) \rightarrow$ $\mathrm{RC}\left(\lambda^{t} ; R^{t}\right)$ of Definition-Proposition 4.1 and Theorem 9.1 below.

\section{Maps on ReCtangles, Littlewood-Richardson tableaux And RIGGED CONFIGURATIONS}

In this section we define several operations on sequences of rectangles and their counterparts on the sets of LR tableaux and rigged configurations. These operations underly the recursive definition of the bijection $\bar{\phi}_{R}: \operatorname{CLR}(\lambda ; R) \rightarrow \operatorname{RC}\left(\lambda^{t} ; R^{t}\right)$ as given in Definition-Proposition 4.1. A summary of the definitions and results of this section is given in Table 1 .

3.1. Operations on sequences of rectangles. Let $R=\left(R_{1}, R_{2}, \ldots, R_{L}\right)$ be a sequence of rectangles such that $R_{j}=\left(\eta_{j}^{\mu_{j}}\right)$ has $\mu_{j}$ rows and $\eta_{j}$ columns. Let $R^{\wedge}$ be the sequence of rectangles obtained from $R$ by splitting off the last column of $R_{L}$; formally, $R_{j}^{\wedge}=R_{j}$ for $1 \leq j \leq L-1, R_{L}^{\wedge}=\left(\left(\eta_{L}-1\right)^{\mu_{L}}\right)$ and $R_{L+1}^{\wedge}=\left(1^{\mu_{L}}\right)$. Note that if the last rectangle of $R$ is a single column, 
then (ignoring the empty rectangle) $R^{\wedge}=R$. If the last rectangle of $R$ is a single column, let $\bar{R}$ be given by removing one cell from the column $R_{L}$; $\bar{R}_{j}=R_{j}$ for $1 \leq j \leq L-1$ and $\bar{R}_{L}=\left(1^{\mu_{L}-1}\right)$. Let $R^{\vee}$ be given by splitting off the first column of $R_{1}$; if $R_{1}$ is a single column, then $R^{\vee}=R$. If $R_{1}$ is a single column, let $\widetilde{R}$ be given by removing one cell from the column $R_{1}$. Finally, let $R^{\mathrm{ev}}=\left(R_{L}, \ldots, R_{2}, R_{1}\right)$ denote the reverse of $R$.

Remark 3.1. Given any sequence of rectangles, there is a unique sequence of transformations of the form $R \rightarrow R^{\wedge}$ or $R \rightarrow \bar{R}$ resulting in the empty sequence, where $R \rightarrow R^{\wedge}$ is only used when the last rectangle of $R$ has more than one column.

3.2. Maps between sets of LR tableaux. For each operation on sequences of rectangles, there is a natural (injective) map on the corresponding sets of LR tableaux of a fixed shape.

Observe that there are inclusions

$$
\begin{aligned}
& \imath^{\wedge}: \operatorname{CLR}(\lambda ; R) \rightarrow \operatorname{CLR}\left(\lambda ; R^{\wedge}\right) \\
& \imath^{\vee}: \operatorname{CLR}(\lambda ; R) \rightarrow \operatorname{CLR}\left(\lambda ; R^{\vee}\right)
\end{aligned}
$$

which correspond to the transformations $R \rightarrow R^{\wedge}$ and $R \rightarrow R^{\vee}$ on rectangles.

Recall that $\operatorname{ST}(\lambda)$ denotes the set of standard tableaux of shape $\lambda$ and define $\operatorname{ST}\left(\lambda^{-}\right)=\bigcup_{\rho \lessdot \lambda} \operatorname{ST}(\rho)$, where $\rho$ and $\lambda$ are partitions and $\rho \lessdot \lambda$ means that $\rho \subset \lambda$ and $\lambda / \rho$ is a single cell. There is a bijection

$$
\begin{aligned}
-: \mathrm{ST}(\lambda) & \rightarrow \mathrm{ST}\left(\lambda^{-}\right) \\
S & \mapsto S^{-}
\end{aligned}
$$

where $S^{-}$is the standard tableau obtained by removing the maximum letter from $S$. Obviously $S$ is uniquely determined by its shape and the tableau $S^{-}$. If the last rectangle of $R$ is a single column, write

$$
\operatorname{CLR}\left(\lambda^{-} ; \bar{R}\right)=\bigcup_{\rho \lessdot \lambda} \operatorname{CLR}(\rho ; \bar{R}) .
$$

The following result is an immediate consequence of the definitions.

Proposition 3.2. Suppose the last rectangle of $R$ is a single column.

1. The map (3.1) restricts to an injection $-: \operatorname{CLR}(\lambda ; R) \rightarrow \operatorname{CLR}\left(\lambda^{-} ; \bar{R}\right)$.

2. If $\mu_{L}=1$ then - is bijective.

3. Suppose $\mu_{L}>1$ and $T \in \operatorname{CLR}\left(\lambda^{-} ; \bar{R}\right)$ such that the cell $\lambda / \operatorname{shape}(T)$ is in the $r$-th row. Then $T$ is in the image of - if and only if the cell shape $(T) /$ shape $\left(T^{-}\right)$is in the $r^{\prime}$-th row with $r^{\prime}<r$.

The injection $-: \operatorname{CLR}(\lambda ; R) \rightarrow \operatorname{CLR}\left(\lambda^{-} ; \bar{R}\right)$ corresponds to the operation $R \rightarrow \bar{R}$ on rectangles. Next we describe a dual operation to $S \mapsto S^{-}$giving rise to the analogue of $R \rightarrow \widetilde{R}$ on LR tableaux. 
Fix partitions $\sigma \subset \lambda$ such that the skew shape $\lambda / \sigma$ has two cells. Consider the set of saturated chains in Young's lattice of partitions under inclusion, that have maximum element $\lambda$ and minimum element $\sigma$ :

$$
\mathcal{C}[\sigma, \lambda]:=\{\sigma \lessdot \rho \lessdot \lambda\} .
$$

If $\lambda / \sigma$ is connected (that is, its two cells are adjacent) then $\mathcal{C}[\sigma, \lambda]$ is a singleton, whose intermediate partition $\rho$ is obtained by adjoining the inner of these two cells to $\sigma$ or removing the outer of the two cells from $\lambda$. If $\lambda / \sigma$ is disconnected (that is, its two cells are not adjacent) then $\mathcal{C}[\sigma, \lambda]$ has exactly two elements, whose intermediate partitions are obtained by adjoining either of the two cells to $\sigma$ or removing either of the two cells from $\lambda$. Let $\tau=\tau_{\sigma, \lambda}$ be the involution on $\mathcal{C}[\sigma, \lambda]$ that has order two if $|\mathcal{C}[\sigma, \lambda]|=2$ (and of course must be the identity when $|\mathcal{C}[\sigma, \lambda]|=1)$.

Definition-Proposition 3.3. For $|\lambda|>0$, there is a unique bijection $D$ : $\mathrm{ST}(\lambda) \rightarrow \mathrm{ST}\left(\lambda^{-}\right)$denoted $S \mapsto S^{D}$ such that:

(D1) If $|\lambda|=1$ then $1^{D}=\emptyset$.

(D2) If $|\lambda|=N>1, S \in \mathrm{ST}(\lambda)$, then $S^{D}$ is uniquely defined by the properties that $S^{D-}=S^{-D}$, and the shape of $S^{D}$ is the intermediate partition in the chain

$$
\tau\left(\sigma \lessdot \operatorname{shape}\left(S^{-}\right) \lessdot \lambda\right)
$$

where $\sigma=\operatorname{shape}\left(S^{-D}\right)$.

$S$ is uniquely determined by its shape and the tableau $S^{D}$.

Using the characterization of $D$ in Definition-Proposition 3.3, it can be shown [21] that $D$ is computed by the following well-known tableau algorithm.

Lemma 3.4. For $S \in \mathrm{ST}(\lambda)$ with $|\lambda|=N, S^{D}$ is obtained by removing the number 1 from $S$, subtracting one from each entry, and sliding the resulting skew tableau to partition shape, that is, $S^{D}=P\left(\left.S\right|_{[2, N]}-1\right)$. More generally for any $0 \leq i \leq N$,

$$
S^{D^{i}}=P\left(\left.S\right|_{[i+1, N]}-i\right) .
$$

Say that the index $i$ is a descent of the standard tableau $S$ if $i+1$ appears in a later row in $S$ than $i$ does.

Lemma 3.5. Suppose $S \in \mathrm{ST}(\lambda)$ and $|\lambda|=N \geq 2$. Let $r$ and $r^{\prime}$ be the rows of the cells shape $(S) / \operatorname{shape}\left(S^{D}\right)$ and shape $\left(S^{D}\right) / \operatorname{shape}\left(S^{D^{2}}\right)$. Then 1 is a descent of $S$ if and only if $r^{\prime}<r$.

Proof. The partition shape $\left(S^{D^{2}}\right)$ is calculated by Lemma 3.4 as the shape obtained by sliding the skew tableau $\left.S\right|_{[3, N]}$ to partition shape, first into the cell of $S$ containing the letter 2 (vacating the cell $s$ say) and then into the cell of $S$ containing the letter 1 (vacating $s^{\prime}$ say). Moreover $s=$ shape $(S) / \operatorname{shape}\left(S^{D}\right)$ and $s^{\prime}=\operatorname{shape}\left(S^{D}\right) / \operatorname{shape}\left(S^{D^{2}}\right)$. 
However shape $\left(S^{D^{2}}\right)$ can be calculated another way. Take the two-letter tableau $U=\left.S\right|_{[1,2]}$ and slide it to the southeast into the cells of $\left.S\right|_{[3, N]}$ occupied by 3 , then 4 , etc., producing the skew tableau $V$ say. Then $\operatorname{shape}\left(S^{D^{2}}\right)$ is given by shape $(S)-\operatorname{shape}(V)$. It is clear that the cells of $V$ containing 1 and 2 are $s^{\prime}$ and $s$ respectively. But sliding preserves Knuth equivalence, so 2 is in a later row than 1 in $U$ (or $S$ ) if and only if it is in $V$.

Suppose the first rectangle of $R$ is a single column. Define

$$
\operatorname{CLR}\left(\lambda^{-} ; \widetilde{R}\right)=\bigcup_{\rho \lessdot \lambda} \operatorname{CLR}(\rho ; \widetilde{R}) .
$$

The analogue of $R \rightarrow \widetilde{R}$ is given by the following proposition.

Proposition 3.6. Suppose the first rectangle of $R$ is a single column.

1. The bijection $D: \mathrm{ST}(\lambda) \rightarrow \mathrm{ST}\left(\lambda^{-}\right)$restricts to an injection $D:$ $\operatorname{CLR}(\lambda ; R) \rightarrow \operatorname{CLR}\left(\lambda^{-} ; \widetilde{R}\right)$.

2. If $\mu_{1}=1$ then $D$ is bijective.

3. Suppose that $\mu_{1}>1$ and $T \in \operatorname{CLR}\left(\lambda^{-} ; \widetilde{R}\right)$ such that the cell $\lambda / \operatorname{shape}(T)$ is in the $r$-th row. Then $T$ is in the image of $D$ if and only if the cell shape $(T) / \operatorname{shape}\left(T^{D}\right)$ is in the $r^{\prime}$-th row with $r^{\prime}<r$.

Proof. 1 follows by Lemma 3.4, the definition of CLR, and the fact that restriction to subintervals preserves Knuth equivalence. For 2 and 3, let $T \in \operatorname{CLR}\left(\lambda^{-} ; \widetilde{R}\right)$. If there is a tableau $S \in \operatorname{CLR}(\lambda ; R)$ such that $T=S^{D}$, then by Lemma $3.4 S$ is obtained from $T$ by sliding $T+1$ to the southeast into the cell $\lambda / \operatorname{shape}(T)$, and placing a 1 in the northwest corner. If $\mu_{1}=1$ then immediately $S \in \operatorname{CLR}(\lambda ; R)$, proving 2 . If $\mu_{1}>1$ then by construction $S \in \operatorname{CLR}(\lambda ; R)$ if and only if 1 is a descent of $S$. By Lemma 3.5 point 3 follows.

Let us discuss carefully the commutation of - and $D$ in (D2) in DefinitionProposition 3.3. Define

$$
\mathrm{ST}\left(\lambda^{--}\right)=\bigcup_{\sigma \lessdot \rho \lessdot \lambda} \mathrm{ST}(\sigma),
$$

where the right hand side is a disjoint union. By definition, for each chain $\mathcal{C}=(\sigma \lessdot \rho \lessdot \lambda)$, there is a distinguished copy of $\operatorname{ST}(\sigma)$ in $\operatorname{ST}\left(\lambda^{--}\right)$denoted $\mathrm{ST}(\sigma)_{\mathcal{C}}$, and for $T \in \mathrm{ST}(\sigma)$, denote its copy in $\mathrm{ST}(\sigma)_{\mathcal{C}}$ by $T_{\mathcal{C}}$. The point is that when $\lambda / \sigma$ is disconnected there are two copies of $\operatorname{ST}(\sigma)$ in $\operatorname{ST}\left(\lambda^{--}\right)$ and they are distinguished by the chain $\mathcal{C}$. By abuse of notation let $\tau$ denote the involution on $\mathrm{ST}\left(\lambda^{--}\right)$that sends $T_{\mathcal{C}} \rightarrow T_{\tau(\mathcal{C})}$. In this notation, which concerns itself with the intermediate partition in the chain $\mathcal{C}$, (D2) is expressed as

$$
-\circ D=\tau \circ D \circ-
$$

viewed as maps $\mathrm{ST}(\lambda) \rightarrow \mathrm{ST}\left(\lambda^{--}\right)$. 
Suppose both the first and last rectangles of $R$ are single columns. Of course $\widetilde{\bar{R}}=\overline{\widetilde{R}}$. Define

$$
\operatorname{CLR}\left(\lambda^{--} ; \widetilde{\bar{R}}\right)=\bigcup_{\sigma \lessdot \rho \lessdot \lambda} \operatorname{CLR}(\sigma ; \widetilde{\bar{R}}) .
$$

The identity of maps (3.3) restricts to the identity of maps $\operatorname{CLR}(\lambda ; R) \rightarrow$ $\operatorname{CLR}\left(\lambda^{--} ; \widetilde{\bar{R}}\right)$ :

$$
-\circ D=\tau \circ D \circ-
$$

Define Schützenberger's evacuation map

$$
\begin{aligned}
\mathrm{ev}: \mathrm{ST}(\lambda) & \rightarrow \mathrm{ST}(\lambda) \\
S & \mapsto S^{\mathrm{ev}}
\end{aligned}
$$

where $S^{\mathrm{ev}} \in \mathrm{ST}(\lambda)$ is defined by $S^{\mathrm{ev}}=S$ if $\lambda=\emptyset$ and $S^{\mathrm{ev}-}=S^{D \mathrm{ev}}$.

The following result is well-known and easy to prove.

Proposition 3.7. ev is an involution satisfying

$$
\text { ev } \circ-=D \circ \mathrm{ev} \quad \text { and } \quad \text { ev } \circ D=-\circ \mathrm{ev} .
$$

Lemma 3.8. Let $|\lambda|=N$ and $S \in \mathrm{ST}(\lambda)$. Then for any $1 \leq i \leq j \leq N$,

$$
P\left(\left.S^{\mathrm{ev}}\right|_{[i, j]}-(i-1)\right)=P\left(\left.S\right|_{[N+1-j, N+1-i]}-(N-j)\right)^{\mathrm{ev}} .
$$

Proof. By Lemma 3.4, (3.3), and the definitions,

$$
\begin{aligned}
P\left(\left.S\right|_{[i, j]}-(i-1)\right) & =P\left(\left.\left(\left.S\right|_{[1, j]}\right)\right|_{[i, j]}-(i-1)\right) \\
& =S^{-N-j} D^{i-1}=S^{D^{i-1}-^{N-j}} .
\end{aligned}
$$

Using this and Proposition 3.7, we have

$$
\begin{aligned}
P\left(\left.S^{\mathrm{ev}}\right|_{[i, j]}-(i-1)\right) & =S^{\mathrm{ev} D^{i-1}-^{N-j}}=S^{-^{i-1} D^{N-j} \mathrm{ev}} \\
& =P\left(\left.S\right|_{[N+1-j, N+1-i]}-(N-j)\right)^{\mathrm{ev}} .
\end{aligned}
$$

Observe that the tableau in the singleton set CLR $\left(R_{1},\left(R_{1}\right)\right)$ evacuates to itself. Using this fact, induction, Lemma 3.8, the definition of CLR, and the fact that Knuth equivalence is preserved under restriction to subintervals, it follows that the evacuation map (3.5) restricts to a bijection

$$
\text { ev }: \operatorname{CLR}(\lambda ; R) \rightarrow \operatorname{CLR}\left(\lambda ; R^{\mathrm{ev}}\right) .
$$

Of course $R^{\wedge \mathrm{ev}}=\left(R^{\mathrm{ev}}\right)^{\vee}$. By definition the following diagram commutes

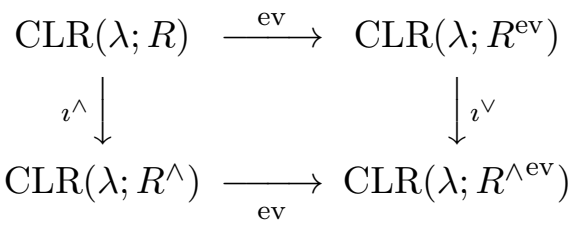


where $\imath^{\vee}$ is defined with respect to the sequence of rectangles $R^{\text {ev }}$. Since ev is an involution, one may exchange the roles of $\imath^{\wedge}$ and $\imath^{\vee}$, of $R^{\wedge}$ and $R^{\vee}$, and of $R$ and $R^{\mathrm{ev}}$.

3.3. Maps between sets of rigged configurations. For the various transformations of sequences of rectangles, one has the following maps between the corresponding sets of rigged configurations.

Define the map

$$
\jmath^{\wedge}: \operatorname{RC}\left(\lambda^{t} ; R^{t}\right) \rightarrow \operatorname{RC}\left(\lambda^{t} ;\left(R^{\wedge}\right)^{t}\right)
$$

by declaring that $\jmath^{\wedge}(\nu, J)$ is obtained from $(\nu, J) \in \operatorname{RC}\left(\lambda^{t} ; R^{t}\right)$ by adding a singular string of length $\mu_{L}$ to each of the first $\eta_{L}-1$ rigged partitions. Note that $\jmath^{\wedge}$ is the identity map if $R_{L}$ is a single column.

Lemma 3.9. $\jmath^{\wedge}$ is a well-defined injection that preserves the vacancy numbers of the underlying configurations.

Proof. Let $\left(\nu^{\wedge}, J^{\wedge}\right)=\jmath^{\wedge}(\nu, J)$. It is enough to show that $\nu^{\wedge}$ is a $\left(\lambda^{t} ;\left(R^{\wedge}\right)^{t}\right)$ configuration that has the same vacancy numbers as $\nu$. First we verify that the partitions in $\nu^{\wedge}$ have the correct size. To this end set $\chi($ true $)=1$ and $\chi($ false $)=0$. Then

$$
\begin{aligned}
\left|\nu^{\wedge(k)}\right|= & \chi\left(k<\eta_{L}\right) \mu_{L}+\left|\nu^{(k)}\right| \\
= & \chi\left(k<\eta_{L}\right) \mu_{L}+\sum_{j>k} \lambda_{j}^{t}-\sum_{a=1}^{L} \mu_{a} \max \left(\eta_{a}-k, 0\right) \\
= & \sum_{j>k} \lambda_{j}^{t}-\sum_{a=1}^{L-1} \mu_{a} \max \left(\eta_{a}-k, 0\right) \\
& -\mu_{L}\left\{\max \left(\eta_{L}-1-k, 0\right)+\max (1-k, 0)\right\} .
\end{aligned}
$$

Next we check that the vacancy numbers remain the same. Note that

$$
Q_{n}\left(\nu^{\wedge(k)}\right)-Q_{n}\left(\nu^{(k)}\right)=\chi\left(1 \leq k<\eta_{L}\right) \min \left(\mu_{L}, n\right),
$$

valid for $k \geq 0$. Then for $k, n \geq 1$,

$$
\begin{aligned}
& P_{n}^{(k)}\left(\nu^{\wedge}\right)-P_{n}^{(k)}(\nu) \\
= & \min \left(\mu_{L}, n\right)\left\{\chi\left(1 \leq k-1<\eta_{L}\right)-2 \chi\left(1 \leq k<\eta_{L}\right)+\chi\left(1 \leq k+1<\eta_{L}\right)\right\} \\
& \quad+\min \left(\mu_{L}, n\right)\left(\delta_{\eta_{L}-1, k}+\delta_{1, k}-\delta_{\eta_{L}, k}\right) \\
= & 0
\end{aligned}
$$

where $\delta_{a, b}=\chi(a=b)$.

Define the map

$$
\jmath^{\vee}: \operatorname{RC}\left(\lambda^{t} ; R^{t}\right) \rightarrow \operatorname{RC}\left(\lambda^{t} ;\left(R^{\vee}\right)^{t}\right)
$$


by declaring that $\jmath^{\vee}(\nu, J)$ is obtained from $(\nu, J) \in \mathrm{RC}\left(\lambda^{t} ; R^{t}\right)$ by adding a string with label zero and length $\mu_{1}$ to each of the first $\eta_{1}-1$ rigged partitions. If $R_{1}$ is a single column then $\jmath^{\vee}$ is the identity.

By Remark 2.11, the proof of Lemma 3.9 also shows that:

Lemma 3.10. $\jmath^{\vee}$ is a well-defined injection that preserves the vacancy numbers of the underlying configurations.

Define the involution

$$
\theta_{R}: \operatorname{RC}\left(\lambda^{t} ; R^{t}\right) \rightarrow \operatorname{RC}\left(\lambda^{t} ; R^{t}\right)
$$

by $\theta_{R}(\nu, J)=(\nu, P-J)$, which is shorthand for saying that $\theta_{R}$ preserves the underlying configuration and replaces the label $x$ of a string $(n, x) \in(\nu, J)^{(k)}$ by its colabel, which is by definition the number $P_{n}^{(k)}(\nu)-x$, its complement with respect to the vacancy number of the string. In light of Remark 2.11, one may also define

$$
\theta_{R}^{\mathrm{ev}}: \mathrm{RC}\left(\lambda^{t} ; R^{t}\right) \rightarrow \mathrm{RC}\left(\lambda^{t} ; R^{\mathrm{ev} t}\right)
$$

which complements the quantum numbers as $\theta_{R}$ and in addition reverses the sequence of rectangles.

By definition the following diagram commutes

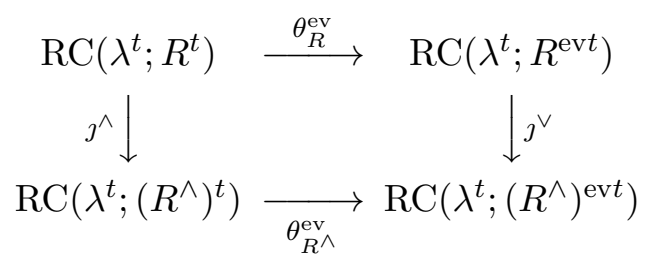

where $\jmath^{\vee}$ acts with respect to the reversed sequence of rectangles $R^{\text {ev }}$. Since $\theta_{R}^{\text {ev }}$ is an involution one may exchange the roles of $\jmath^{\wedge}$ and $\jmath^{\vee}$, of $R^{\wedge}$ and $R^{\vee}$, and of $R$ and $R^{\mathrm{ev}}$.

Suppose the last rectangle of $R$ is a single column. Define the set

$$
\mathrm{RC}\left(\lambda^{-t} ; \bar{R}^{t}\right)=\bigcup_{\rho \lessdot \lambda} \mathrm{RC}\left(\rho^{t} ; \bar{R}^{t}\right) .
$$

The key algorithm on rigged configurations is given by the map

$$
\bar{\delta}: \mathrm{RC}\left(\lambda^{t} ; R^{t}\right) \rightarrow \mathrm{RC}\left(\lambda^{-t} ; \bar{R}^{t}\right),
$$

defined as follows. Let $(\nu, J) \in \mathrm{RC}\left(\lambda^{t} ; R^{t}\right)$. Define $\bar{\ell}^{(0)}=\mu_{L}$. By induction select the singular string in $(\nu, J)^{(k)}$ whose length $\bar{\ell}^{(k)}$ is minimal such that $\bar{\ell}^{(k-1)} \leq \bar{\ell}^{(k)}$. Let $\overline{\mathrm{rk}}(\nu, J)$ denote the smallest $k$ for which no such string exists, and set $\bar{\ell}^{(k)}=\infty$ for $k \geq \overline{\operatorname{rk}}(\nu, J)$. Then $\bar{\delta}(\nu, J)=(\bar{\nu}, \bar{J})$ is obtained from $(\nu, J)$ by shortening each of the selected singular strings by one, changing their labels so that they remain singular, and leaving the other strings unchanged. 
Let us compute the change in vacancy numbers under $\bar{\delta}$. Recalling that $\nu^{(0)}=\emptyset$, observe that

$$
Q_{n}\left(\nu^{(k)}\right)-Q_{n}\left(\bar{\nu}^{(k)}\right)=\chi(k \geq 1) \chi\left(n \geq \bar{\ell}^{(k)}\right)
$$

for $k \geq 0$. Then for $k \geq 2$ and $n \geq 0$ we have

$$
\begin{aligned}
P_{n}^{(k)}(\nu)-P_{n}^{(k)}(\bar{\nu}) & =\chi\left(n \geq \bar{\ell}^{(k-1)}\right)-2 \chi\left(n \geq \bar{\ell}^{(k)}\right)+\chi\left(n \geq \bar{\ell}^{(k+1)}\right) \\
& =\chi\left(\bar{\ell}^{(k-1)} \leq n<\bar{\ell}^{(k)}\right)-\chi\left(\bar{\ell}^{(k)} \leq n<\bar{\ell}^{(k+1)}\right) .
\end{aligned}
$$

Recall that $\bar{\ell}^{(0)}=\mu_{L}$. For $k=1$ and $n \geq 0$,

$$
\begin{aligned}
P_{n}^{(1)}(\nu)-P_{n}^{(1)}(\bar{\nu}) & =-2 \chi\left(n \geq \bar{\ell}^{(1)}\right)+\chi\left(n \geq \bar{\ell}^{(2)}\right)+\chi\left(n \geq \mu_{L}\right) \\
& =\chi\left(\bar{\ell}^{(0)} \leq n<\bar{\ell}^{(1)}\right)-\chi\left(\bar{\ell}^{(1)} \leq n<\bar{\ell}^{(2)}\right) .
\end{aligned}
$$

Therefore for all $k \geq 1$ and $n \geq 0$ we have

$$
P_{n}^{(k)}(\nu)-P_{n}^{(k)}(\bar{\nu})=\chi\left(\bar{\ell}^{(k-1)} \leq n<\bar{\ell}^{(k)}\right)-\chi\left(\bar{\ell}^{(k)} \leq n<\bar{\ell}^{(k+1)}\right) .
$$

As before $m_{n}(\rho)$ denotes the number of parts of size $n$ in the partition $\rho$. One may easily verify (see also [13, Appendix]) that for all $k, n \geq 1$

$$
\begin{aligned}
& -P_{n-1}^{(k)}(\nu)+2 P_{n}^{(k)}(\nu)-P_{n+1}^{(k)}(\nu) \\
= & m_{n}\left(\nu^{(k-1)}\right)-2 m_{n}\left(\nu^{(k)}\right)+m_{n}\left(\nu^{(k+1)}\right)+m_{n}\left(\xi^{(k)}(R)\right) \\
\geq & m_{n}\left(\nu^{(k-1)}\right)-2 m_{n}\left(\nu^{(k)}\right)+m_{n}\left(\nu^{(k+1)}\right) .
\end{aligned}
$$

In particular the vacancy numbers have the partial convexity property (see [13, (11.1)])

$$
P_{n}^{(k)}(\nu) \geq 1 / 2\left(P_{n-1}^{(k)}(\nu)+P_{n+1}^{(k)}(\nu)\right) \quad \text { if } m_{n}\left(\nu^{(k)}\right)=0 .
$$

Repeated use of (3.11) leads to the following result.

Lemma 3.11. Let $\nu$ be an admissible configuration and $m_{n}\left(\nu^{(k)}\right)=0$ for $a<n<b$.

1. $P_{n}^{(k)}(\nu) \geq \min \left(P_{a}^{(k)}(\nu), P_{b}^{(k)}(\nu)\right)$ for $a \leq n \leq b$.

2. If $P_{c}^{(k)}(\nu)=0$ for some $a<c<b$ then $P_{n}^{(k)}(\nu)=0$ for all $a \leq n \leq b$.

3. If $P_{c}^{(k)}(\nu)=P_{c+1}^{(k)}(\nu)=1$ for some $c$ such that $a \leq c<b$ then $P_{n}^{(k)}(\nu)=$ 1 for all $a<n<b$.

The following proposition is important for the definition of the bijection $\bar{\phi}_{R}$ between rigged configurations and LR tableaux to be defined in Section 田.

Proposition 3.12. Let $(\nu, J) \in \mathrm{RC}\left(\lambda^{t} ; R^{t}\right)$ where the last rectangle of $R$ is a single column.

1. The map $\bar{\delta}$ is a well-defined injection such that $\bar{\delta}(\nu, J) \in \operatorname{RC}\left(\rho^{t} ; \bar{R}^{t}\right)$ where $\rho$ is obtained from $\lambda$ by removing the corner cell in the column of index $\overline{\operatorname{rk}}(\nu, J)$. 
2. If $\mu_{L}=1$ then $\bar{\delta}$ is bijective.

3. If $\mu_{L}>1$ then $\left(\nu^{\prime}, J^{\prime}\right) \in \mathrm{RC}\left(\rho^{t} ; \bar{R}^{t}\right)$ is in the image of $\bar{\delta}$ if and only if $\overline{\mathrm{rk}}\left(\nu^{\prime}, J^{\prime}\right) \geq \overline{\mathrm{rk}}(\nu, J)$.

Proof. To prove that $\bar{\delta}$ is well-defined it needs to be shown that $(\bar{\nu}, \bar{J})=$ $\bar{\delta}(\nu, J)$ is an admissible rigged $\left(\rho^{t} ; \bar{R}^{t}\right)$-configuration. Let us first show that $\rho$ obtained from $\lambda$ by removing the corner cell in column of index $\overline{\mathrm{rk}}(\nu, J)$ is indeed a partition. Assume the contrary. This means that $\lambda_{r}^{t}=\lambda_{r+1}^{t}$ where $r=\overline{\operatorname{rk}}(\nu, J)$. By [13, (11.2)] $P_{n}^{(k)}(\nu)=\lambda_{k}^{t}-\lambda_{k+1}^{t}$ for large $n$, so that $P_{n}^{(r)}(\nu)=0$. Let $\ell$ be the size of the largest part of $\nu^{(r)}$. Then by Lemma 3.11 it follows that $P_{n}^{(r)}(\nu)=0$ for all $n \geq \ell$. Since $m_{n}\left(\nu^{(r)}\right)=0$ for $n>\ell$ and $P_{n}^{(k)}(\nu) \geq 0$ for all $k \geq 1, n \geq 0$, inequality (3.10) implies in particular that $m_{n}\left(\nu^{(r-1)}\right)=0$ for $n>\ell$. This means that $1 \leq \bar{\ell}^{(r-1)} \leq \ell$. Since $P_{\ell}^{(r)}(\nu)=0$ and $m_{\ell}\left(\nu^{(r)}\right)>0$ there is a singular string of length $\ell$ in $\nu^{(r)}$ weakly bigger than $\bar{\ell}^{(r-1)}$. However, this contradicts the assumption that $r=\overline{\mathrm{rk}}(\nu, J)$ which would imply that there is no such singular string. Hence $\rho$ is a partition. By the definition of $\overline{\operatorname{rk}}(\nu, J)$ it is clear that $\left|\bar{\nu}^{(k)}\right|=$ $\left|\nu^{(k)}\right|-\chi(k<\overline{\operatorname{rk}}(\nu, J))$. Since $\eta_{L}=1$ it follows from (2.4) that $\bar{\nu}$ is a $\left(\rho^{t} ; \bar{R}^{t}\right)$-configuration.

Next we need to show that $(\bar{\nu}, \bar{J})$ is admissible. Denote by $J_{n}^{(k)}(\nu, J)$ the maximal rigging occurring in the strings of length $n$ in $(\nu, J)^{(k)}$ (which is set to zero if $n$ does not appear as a part in $\left.\nu^{(k)}\right)$. Then to prove the admissibility of $(\bar{\nu}, \overline{\mathrm{J}})$ we need to show for all $n, k \geq 1$ that

$$
0 \leq J_{n}^{(k)}(\bar{\nu}, \overline{\mathrm{J}}) \leq P_{n}^{(k)}(\bar{\nu}) .
$$

Fix $k \geq 1$. Since only one string of size $\bar{\ell}^{(k)}$ changes in the transformation $(\nu, J)^{(k)} \rightarrow(\bar{\nu}, \bar{J})^{(k)}$ one finds that $J_{n}^{(k)}(\bar{\nu}, \bar{J})=J_{n}^{(k)}(\nu, J)$ for $1 \leq n<\bar{\ell}^{(k)}-1$, $J_{n}^{(k)}(\bar{\nu}, \bar{J})=P_{n}^{(k)}(\bar{\nu})$ for $n=\bar{\ell}^{(k)}-1$ and $0 \leq J_{n}^{(k)}(\bar{\nu}, \bar{J}) \leq J_{n}^{(k)}(\nu, J)$ for $n \geq \bar{\ell}^{(k)}$. Hence by (3.9) the inequality (3.12) can only be violated when $\bar{\ell}^{(k-1)} \leq n<\bar{\ell}^{(k)}$. By the construction of $\bar{\ell}^{(k)}$ there are no singular strings of length $n$ in $(\nu, J)^{(k)}$ for $\bar{\ell}^{(k-1)} \leq n<\bar{\ell}^{(k)}$. This means that $J_{n}^{(k)}(\nu, J) \leq$ $P_{n}^{(k)}(\nu)-1$ if $n$ occurs as a part in $\nu^{(k)}$, that is $m_{n}\left(\nu^{(k)}\right)>0$. Hence due to $(3.9)$ the condition (3.12) is fulfilled for these $n$. It remains to prove that $P_{n}^{(k)}(\bar{\nu}) \geq 0$ for all $n$ such that $m_{n}\left(\nu^{(k)}\right)=0$ and $\bar{\ell}^{(k-1)} \leq n<\bar{\ell}^{(k)}$. Note that $m_{n}\left(\bar{\nu}^{(k)}\right)=0$ if $m_{n}\left(\nu^{(k)}\right)=0$ for $\bar{\ell}^{(k-1)} \leq n<\bar{\ell}^{(k)}-1$. By 13, Lemma 10] it suffices to prove (3.12) for all $k$ and $n$ such that $m_{n}\left(\bar{\nu}^{(k)}\right)>0$. Therefore the only remaining case for which (3.12) might be violated occurs when

$$
m_{\ell-1}\left(\nu^{(k)}\right)=0, \quad P_{\ell-1}^{(k)}(\nu)=0, \quad \bar{\ell}^{(k-1)}<\ell \quad \text { and } \ell \text { finite }
$$


where $\ell=\bar{\ell}^{(k)}$. We show that these conditions cannot be met simultaneously. Let $p<\ell$ be maximal such that $m_{p}\left(\nu^{(k)}\right)>0$; if no such $p$ exists set $p=0$. By Lemma $3.11 P_{\ell-1}^{(k)}(\nu)=0$ is only possible if $P_{n}^{(k)}(\nu)=0$ for all $p \leq n \leq \ell$. By (3.10) we find that $m_{n}\left(\nu^{(k-1)}\right)=0$ for $p<n<\ell$. Since $\bar{\ell}^{(k-1)}<\ell$ this implies that $\bar{\ell}^{(k-1)} \leq p$. If $p=0$ this contradicts the condition $\bar{\ell}^{(k-1)} \geq 1$. Hence assume that $p>0$. Since $P_{p}^{(k)}(\nu)=0$ and $m_{p}\left(\nu^{(k)}\right)>0$ there is a singular string of length $p$ in $(\nu, J)^{(k)}$ and therefore $\bar{\ell}^{(k)}=p$. However, this contradicts $p<\ell=\bar{\ell}^{(k)}$. This concludes the proof of the admissibility of $(\bar{\nu}, \bar{J})$ and also the proof of the well-definedness of $\bar{\delta}$.

For the proof of the injectivity of $\bar{\delta}$, and points 2 and 3 we require the algorithm $\bar{\delta}^{-1}$ defined on $\operatorname{RC}\left(\lambda^{-t} ; \bar{R}^{t}\right)$ as follows. Recall that $(\bar{\nu}, \bar{J}) \in$ $\mathrm{RC}\left(\lambda^{-t} ; \bar{R}^{t}\right)$ means that $(\bar{\nu}, \overline{\mathrm{J}}) \in \mathrm{RC}\left(\rho^{t} ; \bar{R}^{t}\right)$ for some $\rho \lessdot \lambda$. Suppose that the cell $\lambda / \rho$ has column index $c$ in $\lambda$. Set $\bar{s}^{(k)}=\infty$ for $k \geq c$. For $1 \leq k<c$ select by downward induction the singular string in $(\bar{\nu}, \overline{\bar{J}})^{(k)}$ whose length $\bar{s}^{(k)}$ is maximal such that $\bar{s}^{(k)} \leq \bar{s}^{(k+1)}$; set $\bar{s}^{(k)}=0$ if no such string exists. Then $(\nu, J)=\bar{\delta}^{-1}(\bar{\nu}, \overline{\mathrm{J}})$ is obtained from $(\bar{\nu}, \overline{\mathrm{J}})$ by adding one box to the selected strings (and adding a string of length one if $\bar{s}^{(k)}=0$ ) with labels such that they remain singular, and leaving all other strings unchanged.

It is obvious from the constructions and (3.9) that for $(\nu, J) \in \mathrm{RC}\left(\lambda^{t} ; R^{t}\right)$, $\bar{\delta}^{-1} \circ \bar{\delta}(\nu, J)=(\nu, J)$ since $\bar{s}^{(k)}=\bar{\ell}^{(k)}-1$. This proves that $\bar{\delta}$ is an injection and concludes the proof of point 1 .

It follows immediately from the definition of $\bar{\delta}$ that $\overline{\mathrm{rk}}(\bar{\delta}(\nu, J)) \geq \overline{\mathrm{rk}}(\nu, J)$ for $(\nu, J) \in \operatorname{RC}\left(\lambda^{t} ; R^{t}\right)$ where $\mu_{L}>1$. Hence, if $\left(\nu^{\prime}, J^{\prime}\right) \in \operatorname{RC}\left(\rho^{t} ; \bar{R}^{t}\right)$ is in the image of $\bar{\delta}$, then $\overline{\operatorname{rk}}\left(\nu^{\prime}, J^{\prime}\right) \geq \overline{\operatorname{rk}}(\nu, J)$. To prove the reverse and point 2 we introduce the set $\mathrm{RC}^{\prime}\left(\lambda^{-t} ; \bar{R}^{t}\right) \subset \mathrm{RC}\left(\lambda^{-t} ; \bar{R}^{t}\right)$ as follows. For $\mu_{L}=1$ set $\mathrm{RC}^{\prime}\left(\lambda^{-t} ; \bar{R}^{t}\right)=\operatorname{RC}\left(\lambda^{-t} ; \bar{R}^{t}\right)$. For $\mu_{L}>1,(\bar{\nu}, \bar{J}) \in \operatorname{RC}^{\prime}\left(\lambda^{-t} ; \bar{R}^{t}\right)$ if $\overline{\operatorname{rk}}(\bar{\nu}, \bar{J}) \geq c$ where $c$ is the column index of the cell $\lambda / \rho$ and $\rho \lessdot \lambda$ is the partition corresponding to $(\bar{\nu}, \overline{\mathrm{J}})$.

It will be shown that

$$
\bar{\delta}^{-1}: \mathrm{RC}^{\prime}\left(\lambda^{-t} ; \bar{R}^{t}\right) \rightarrow \mathrm{RC}\left(\lambda^{t} ; R^{t}\right)
$$

is well-defined. Then set $(\nu, J)=\bar{\delta}^{-1}(\bar{\nu}, \bar{J})$ for $(\bar{\nu}, \bar{J}) \in \mathrm{RC}^{\prime}\left(\lambda^{-t} ; \bar{R}^{t}\right)$. Notice that the condition $\overline{\mathrm{rk}}(\bar{\nu}, \overline{\mathrm{J}}) \geq c$ implies that $\bar{\ell}^{(k)} \leq \bar{s}^{(k)}$ where $\bar{\ell}^{(k)}$ and $\bar{s}^{(k)}$ are the lengths of the selected strings in $(\bar{\nu}, \bar{J})$ under $\bar{\delta}$ and $\bar{\delta}^{-1}$, respectively. In particular, $\bar{\ell}^{(0)} \leq \bar{\ell}^{(1)} \leq \bar{s}^{(1)}$ so that $\bar{s}^{(0)}:=\mu_{L}-1 \leq \bar{s}^{(1)}$. Using this one may easily verify that for all $k \geq 1$ and $n \geq 0$ the change in vacancy numbers under $\bar{\delta}^{-1}$ is given by

$$
P_{n}^{(k)}(\nu)-P_{n}^{(k)}(\bar{\nu})=\chi\left(\bar{s}^{(k-1)}<n \leq \bar{s}^{(k)}\right)-\chi\left(\bar{s}^{(k)}<n \leq \bar{s}^{(k+1)}\right) .
$$


It follows from the constructions of $\bar{\delta}$ and $\bar{\delta}^{-1}$ and (3.13) that $\bar{\delta} \circ \bar{\delta}^{-1}(\bar{\nu}, \bar{J})=$ $(\bar{\nu}, \bar{J})$ for $(\bar{\nu}, \bar{J}) \in \mathrm{RC}^{\prime}\left(\lambda^{-t} ; \bar{R}^{t}\right)$. This implies that the image of $\bar{\delta}$ is given by $\mathrm{RC}^{\prime}\left(\lambda^{-t} ; \bar{R}^{t}\right)$ proving point 3 . Since for $\mu_{L}=1, \mathrm{RC}^{\prime}\left(\lambda^{-t} ; \bar{R}^{t}\right)=\operatorname{RC}\left(\lambda^{-t} ; \bar{R}^{t}\right)$ it follows that in this case $\bar{\delta}$ is a bijection proving point 2 .

We are left to prove that $\bar{\delta}^{-1}$ is well-defined, that is, for every $(\bar{\nu}, \bar{J}) \in$ $\mathrm{RC}^{\prime}\left(\lambda^{-t} ; \bar{R}^{t}\right)$ the rigged configuration $(\nu, J)=\bar{\delta}^{-1}(\bar{\nu}, \bar{J})$ is admissible. This can be shown in a very similar fashion to the proof of the well-definedness of $\bar{\delta}$. Hence we only highlight the main arguments. By construction there are no singular strings of length $n$ in $(\bar{\nu}, \overline{\mathrm{J}})^{(k)}$ for $\bar{s}^{(k)}<n \leq \bar{s}^{(k+1)}$. Hence by (3.13) and [13, Lemma 10] we need to show this time that for $\ell=\bar{s}^{(k)}$ the conditions

$$
m_{\ell+1}\left(\bar{\nu}^{(k)}\right)=0, \quad P_{\ell+1}^{(k)}(\bar{\nu})=0, \quad \ell<\bar{s}^{(k+1)} \quad \text { and } \ell \text { finite }
$$

cannot all be met simultaneously for all $k \geq 1$. Fix $k \geq 1$. Let $\ell<p$ be minimal such that $m_{p}\left(\bar{\nu}^{(k)}\right)>0$; if no such $p$ exists set $p=\infty$. By Lemma 3.11 the condition $P_{\ell+1}^{(k)}(\bar{\nu})=0$ implies that $P_{n}^{(k)}(\bar{\nu})=0$ for all $\ell \leq n \leq p$. The condition that $\ell$ is finite requires $k<c$ where recall that $c$ is the column index of the cell $\lambda / \rho$.

First assume that $k=c-1$. If $p$ is finite, i.e., $\ell$ is not the largest part in $\bar{\nu}^{(k)}$ then there exists a singular string of length $p$ since $P_{p}^{(k)}(\bar{\nu})=0$ as argued above. But this means $\ell<\bar{s}^{(k)}$ which contradicts our assumptions. Hence assume that $\ell$ is the largest part in $\bar{\nu}^{(k)}$. By [13, (11.2)] one finds that $P_{n}^{(k)}(\bar{\nu})=\rho_{k}^{t}-\rho_{k+1}^{t}$ for large $n$. Since $P_{n}^{(k)}(\bar{\nu})=0$ for $n \geq \ell$ this requires $\rho_{k}^{t}=\rho_{k+1}^{t}$. Since $\lambda$ is a partition this implies that $c \neq k+1$ which contradicts the assumption. Hence (3.14) cannot occur for $k=c-1$.

Now assume that $k<c-1$ which implies that $\bar{s}^{(k+1)}$ is finite. Since $P_{n}^{(k)}(\bar{\nu})=0$ for $\ell \leq n \leq p$ one finds from (3.10) that $m_{n}\left(\bar{\nu}^{(k+1)}\right)=0$ for $\ell<n<p$ which means that $\bar{\nu}^{(k+1)}$ does not contain any parts of size $n$. When $\ell$ is not the largest part in $\bar{\nu}^{(k)}$, that is, $p$ is finite, we conclude that $\bar{s}^{(k+1)} \geq p$. However, since $P_{p}^{(k)}(\bar{\nu})=0$, there exists a singular string of size $p$ in $(\bar{\nu}, \bar{J})^{(k)}$ and hence $\bar{s}^{(k)} \geq p>\ell$. This contradicts $\bar{s}^{(k)}=\ell$. When $\ell$ is the largest part in $\bar{\nu}^{(k)}$, that is, $p$ is infinite, we infer that the largest part in $\bar{\nu}^{(k+1)}$ can be at most of size $\ell$. This implies $\bar{s}^{(k+1)} \leq \ell$ which contradicts the assumption $\ell<\bar{s}^{(k+1)}$. This concludes the proof that the conditions (3.14) cannot occur and shows that $\bar{\delta}^{-1}$ is well-defined.

Suppose the first rectangle of $R$ is a single column. Define the set

$$
\mathrm{RC}\left(\lambda^{-t} ; \widetilde{R}^{t}\right)=\bigcup_{\rho \lessdot \lambda} \mathrm{RC}\left(\rho^{t} ; \widetilde{R}^{t}\right) .
$$


Define the map $\widetilde{\delta}: \mathrm{RC}\left(\lambda^{t} ; R^{t}\right) \rightarrow \mathrm{RC}\left(\lambda^{-t} ; \widetilde{R}^{t}\right)$ such that the diagram commutes:

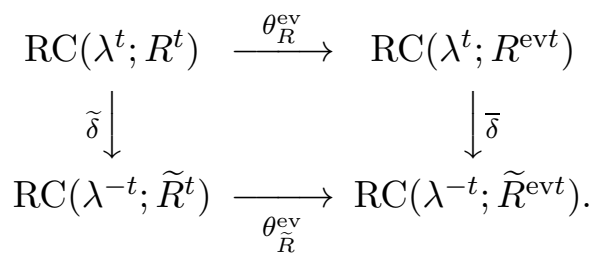

More precisely, for $(\nu, J) \in \operatorname{RC}\left(\lambda^{t} ; R^{\text {evt }}\right)$, let $\bar{\delta}(\nu, J) \in \mathrm{RC}\left(\rho^{t} ; \widetilde{R}^{\text {evt }}\right)$ for $\rho \lessdot \lambda$. Then for $(\nu, J) \in \operatorname{RC}\left(\lambda^{t} ; R^{t}\right), \widetilde{\delta}(\nu, J)=\left(\theta_{\widetilde{R}^{\text {ev }}}^{\text {ev }} \circ \bar{\delta} \circ \theta_{R}^{\text {ev }}\right)(\nu, J) \in \operatorname{RC}\left(\rho^{t} ; \widetilde{R}^{t}\right)$ and define $\widetilde{\operatorname{rk}}(\nu, J)=\overline{\mathrm{rk}}\left(\theta_{R}^{\mathrm{ev}}(\nu, J)\right)$. Observe that by definition and Proposition $3.12 \widetilde{\delta}$ is an injection, with image given by $\left(\nu^{\prime}, J^{\prime}\right) \in \mathrm{RC}\left(\rho^{t} ; \widetilde{R}^{t}\right)$ such that $\widetilde{\operatorname{rk}}\left(\nu^{\prime}, J^{\prime}\right) \geq c$ where $c$ is the column of the cell $\lambda / \rho$. The map $\widetilde{\delta}$ is given explicitly by the following algorithm. Define $\widetilde{\ell}^{(0)}=\mu_{1}$. Inductively select a string in $(\nu, J)^{(k)}$ with label 0 and with length $\tilde{\ell}^{(k)}$ minimal such that $\widetilde{\ell}^{(k-1)} \leq \widetilde{\ell}^{(k)}$. Then $\widetilde{\mathrm{rk}}(\nu, J)$ is the minimum index $k$ for which such a string does not exist; set $\widetilde{\ell}^{(k)}=\infty$ for $k \geq \widetilde{\operatorname{rk}}(\nu, J)$. Then $\widetilde{\delta}(\nu, J)=(\widetilde{\nu}, \widetilde{\mathrm{J}})$ is given by shortening the selected strings by one and keeping their labels zero, and changing the labels on all other strings such that their colabels do not change. The computation for (3.9) yields

$$
P_{n}^{(k)}(\nu)-P_{n}^{(k)}(\widetilde{\nu})=\chi\left(\widetilde{\ell}^{(k-1)} \leq n<\widetilde{\ell}^{(k)}\right)-\chi\left(\widetilde{\ell}^{(k)} \leq n<\widetilde{\ell}^{(k+1)}\right)
$$

for $k \geq 1$ and $n \geq 0$.

Lemma 3.13. Suppose the first and last rectangles of $R$ are single columns. Then $\bar{\delta}$ and $\widetilde{\delta}$ commute. Moreover, for all $(\nu, J) \in \mathrm{RC}\left(\lambda^{t} ; R^{t}\right)$ one of the following conditions holds, and the second can only hold if $r:=\overline{\mathrm{rk}}(\nu, J)=$ $\widetilde{\mathrm{rk}}(\nu, J)$ and $\lambda_{r}^{t}=\lambda_{r-1}^{t}$.

1. $\overline{\mathrm{rk}}(\widetilde{\delta}(\nu, J))=\overline{\mathrm{rk}}(\nu, J)$ and $\widetilde{\mathrm{rk}}(\bar{\delta}(\nu, J))=\widetilde{\mathrm{rk}}(\nu, J)$.

2. $\overline{\mathrm{rk}}(\widetilde{\delta}(\nu, J))=\overline{\mathrm{rk}}(\nu, J)-1$ and $\widetilde{\mathrm{rk}}(\bar{\delta}(\nu, J))=\widetilde{\mathrm{rk}}(\nu, J)-1$.

The proof of Lemma 3.13 is rather technical and is placed in Appendix A. The statement regarding $\overline{\mathrm{rk}}$ and $\widetilde{\mathrm{rk}}$ requires some explanation. Define

$$
\mathrm{RC}\left(\lambda^{--t} ; \widetilde{\bar{R}}^{t}\right)=\bigcup_{\sigma \lessdot \rho \lessdot \lambda} \mathrm{RC}\left(\sigma^{t} ; \widetilde{\bar{R}}^{t}\right) .
$$

Similarly as for $\operatorname{CLR}\left(\lambda^{--} ; \widetilde{\bar{R}}\right)$, define the involution $\tau$ on $\operatorname{RC}\left(\lambda^{--t} ; \widetilde{\bar{R}}^{t}\right)$ by $(\nu, J)_{\mathcal{C}} \mapsto(\nu, J)_{\tau(\mathcal{C})}$. Then Lemma 3.13 says precisely that

$$
\widetilde{\delta} \circ \bar{\delta}=\tau \circ \bar{\delta} \circ \widetilde{\delta}
$$

as maps $\mathrm{RC}\left(\lambda^{t} ; R^{t}\right) \rightarrow \mathrm{RC}\left(\lambda^{--t} ; \widetilde{\bar{R}}^{t}\right)$. 


\begin{tabular}{|l|c|c|}
\hline Rectangles & LR tableaux & Rigged configurations \\
\hline$R \rightarrow R^{\wedge}$ & $\imath^{\wedge}$ & $\jmath^{\wedge}$ \\
$R \rightarrow R^{\vee}$ & $\imath^{\vee}$ & $\jmath^{\vee}$ \\
$R \rightarrow \bar{R}$ & - & $\bar{\delta}$ \\
$R \rightarrow \widetilde{R}$ & $D$ & $\widetilde{\delta}$ \\
$R \rightarrow R^{\mathrm{ev}}$ & $\mathrm{ev}$ & $\theta_{R}^{\mathrm{ev}}$ \\
& $-\circ D=\tau \circ D \circ-$ & $\bar{\delta} \circ \widetilde{\delta}=\tau \circ \widetilde{\delta} \circ \bar{\delta}$ \\
& ev $\circ D=-\circ \mathrm{ev}$ & $\theta_{\widetilde{R}}^{\mathrm{ev}} \circ \widetilde{\delta}=\bar{\delta} \circ \theta_{R}^{\mathrm{ev}}$ \\
& ev $\circ \imath^{\wedge}=\imath^{\vee} \circ \mathrm{ev}$ & $\theta_{R^{\wedge}}^{\mathrm{ev}} \circ \jmath^{\wedge}=\jmath^{\vee} \circ \theta_{R}^{\mathrm{ev}}$ \\
\hline
\end{tabular}

TABLE 1. Maps defined in Section 3 and some of their relations

For the reader's convenience the analogous maps on LR tableaux and rigged configurations as defined in this Section and their main relations are listed in Table 1 .

\section{The Bijection}

4.1. Definition. We require two bijections $\bar{\phi}_{R}$ and $\widetilde{\phi}_{R}$ between LittlewoodRichardson tableaux and rigged configurations. The quantum number bijection $\bar{\phi}_{R}: \operatorname{CLR}(\lambda ; R) \rightarrow \operatorname{RC}\left(\lambda^{t} ; R^{t}\right)$ is defined inductively based on Remark 3.1 in Definition-Proposition 4.1 below. Recall that $\theta_{R}$ complements the quantum numbers of a rigged configuration (see (3.7)). The coquantum number bijection $\widetilde{\phi}_{R}: \operatorname{CLR}(\lambda ; R) \rightarrow \operatorname{RC}\left(\lambda^{t} ; R^{t}\right)$ is defined as

$$
\widetilde{\phi}_{R}:=\theta_{R} \circ \bar{\phi}_{R}
$$

It is $\widetilde{\phi}_{R}$ that preserves the statistics (see Theorem 9.1).

Definition-Proposition 4.1. There is a unique family of bijections $\bar{\phi}_{R}$ : $\operatorname{CLR}(\lambda ; R) \rightarrow \operatorname{RC}\left(\lambda^{t} ; R^{t}\right)$ indexed by $R$, such that:

1. If the last rectangle of $R$ is a single column, then the following diagram commutes:

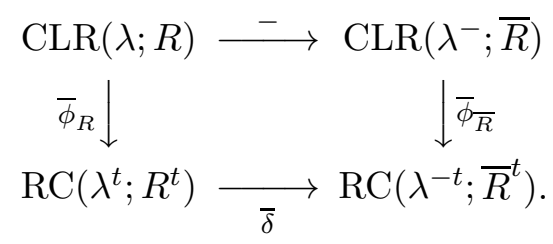


2. The following diagram commutes:

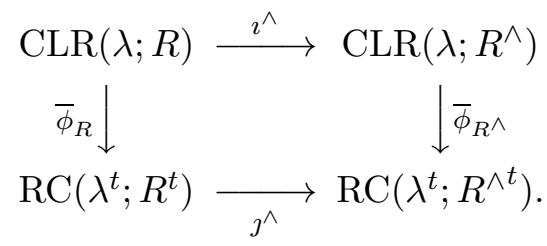

Proof. The proof proceeds by the induction on $R$ given by Remark 3.1. Suppose first that $R$ is the empty sequence. Then both $\operatorname{CLR}(\lambda ; R)$ and $\operatorname{RC}\left(\lambda^{t} ; R^{t}\right)$ are the empty set unless $\lambda$ is the empty partition, in which case $\operatorname{CLR}(\lambda ; R)$ is the singleton consisting of the empty tableau, $\operatorname{RC}\left(\lambda^{t} ; R^{t}\right)$ is the singleton consisting of the empty rigged configuration, and $\bar{\phi}_{\emptyset}$ is the unique bijection $\operatorname{CLR}(\emptyset ; \emptyset) \rightarrow \operatorname{RC}(\emptyset ; \emptyset)$.

Suppose that the last rectangle of $R$ is a single column. Then 2 holds trivially since $R^{\wedge}=R$ and $\imath^{\wedge}$ and $\jmath^{\wedge}$ are the identity maps. Consider 1. By induction, for every partition $\rho$ such that $\rho \lessdot \lambda$ the result holds for the pair $(\rho ; \bar{R})$. Any map $\bar{\phi}_{R}$ satisfying (4.2) is injective by definition and unique by induction. For the existence and surjectivity of $\bar{\phi}_{R}$ it suffices to show that the bijection $\bar{\phi}_{\bar{R}}: \operatorname{CLR}\left(\lambda^{-} ; \bar{R}\right) \rightarrow \operatorname{RC}\left(\lambda^{-t} ; \bar{R}^{t}\right)$ maps the image of - : $\operatorname{CLR}(\lambda ; R) \rightarrow \operatorname{CLR}\left(\lambda^{-} ; \bar{R}\right)$ onto the image of $\bar{\delta}: \operatorname{RC}\left(\lambda^{t} ; R^{t}\right) \rightarrow \operatorname{RC}\left(\lambda^{-t} ; \bar{R}^{t}\right)$. If $\mu_{L}=1$ then - and $\bar{\delta}$ are bijections by Propositions 3.2 and 3.12 respectively proving the assertion. Hence assume $\mu_{L}>1$. Let $c$ and $c^{\prime}$ be the column indices of the cells $\lambda / \rho$ and $\rho / \sigma$ for the partitions $\sigma \lessdot \rho \lessdot \lambda$, respectively. By Proposition 3.2, $T \in \operatorname{CLR}(\rho ; \bar{R})$ with shape $\left(T^{-}\right)=\sigma$ is in the image of $-: \operatorname{CLR}(\lambda ; R) \rightarrow \operatorname{CLR}\left(\lambda^{-} ; \bar{R}\right)$ if and only if $c \leq c^{\prime}$. Similarly by Proposition 3.12, $(\nu, J) \in \mathrm{RC}\left(\rho^{t} ; \bar{R}^{t}\right)$ with $\overline{\mathrm{rk}}(\nu, J)=c^{\prime}$ is in the image of $\bar{\delta}: \mathrm{RC}\left(\lambda^{t} ; R^{t}\right) \rightarrow \mathrm{RC}\left(\lambda^{-t} ; \bar{R}^{t}\right)$ if and only if $c \leq c^{\prime}$. This proves the assertion about the images of - and $\bar{\delta}$.

Suppose the last rectangle of $R$ has more than one column, that is $\eta_{L}>1$. Any map $\bar{\phi}_{R}$ satisfying 2 is injective by definition and unique by induction. For existence and surjectivity it is enough to show that the bijection $\bar{\phi}_{R^{\wedge}}$ maps the image $\mathrm{CLR}^{\wedge}\left(\lambda ; R^{\wedge}\right)$ of $\imath^{\wedge}: \operatorname{CLR}(\lambda ; R) \rightarrow \operatorname{CLR}\left(\lambda ; R^{\wedge}\right)$ onto the image $\mathrm{RC}^{\wedge}\left(\lambda^{t} ; R^{\wedge t}\right)$ of $\jmath^{\wedge}: \mathrm{RC}\left(\lambda^{t} ; R^{t}\right) \rightarrow \mathrm{RC}\left(\lambda^{t} ; R^{\wedge t}\right)$. A rigged configuration $(\nu, J)$ is in $\operatorname{RC}^{\wedge}\left(\lambda^{t} ; R^{\wedge t}\right)$ if and only if $(\nu, J) \in \operatorname{RC}\left(\lambda^{t} ; R^{\wedge t}\right)$ and $(\nu, J)^{(k)}$ contains a singular string of length $\mu_{L}$ for all $1 \leq k<\eta_{L}$. An LR tableau $T$ is in $\operatorname{CLR}^{\wedge}\left(\lambda ; R^{\wedge}\right)$ if and only if $T \in \operatorname{CLR}\left(\bar{\lambda} ; R^{\wedge}\right)$ and the column index $c_{i}$ of the cell shape $\left(T_{i}\right) / \operatorname{shape}\left(T_{i-1}\right)$ and the column index $\bar{c}_{i}$ of the cell shape $\left(\bar{T}_{i}\right) / \operatorname{shape}\left(\bar{T}_{i-1}\right)$ where $T_{i}=T^{-\mu_{L^{-i}}}$ and $\bar{T}_{i}=\left(\imath^{\wedge}\left(T_{0}\right)\right)^{-\mu_{L^{-i}}}$ obey $\bar{c}_{i}<c_{i}$ for all $1 \leq i \leq \mu_{L}$. This follows from the definition of CLR and Remark 2.3.

Set $\left(\nu_{i}, J_{i}\right)=\bar{\delta}^{\mu_{L}-i}(\nu, J)$ for $0 \leq i \leq \mu_{L}, c_{i}=\overline{\operatorname{rk}}\left(\nu_{i}, J_{i}\right)$ and denote the length of the string in $\left(\nu_{i}, J_{i}\right)^{(k)}$ selected by $\bar{\delta}$ by $\ell_{i}^{(k)}$. Similarly set $(\bar{\nu}, \bar{J})=$ $\jmath^{\wedge}\left(\nu_{0}, J_{0}\right)$ and $\left(\bar{\nu}_{i}, \overline{\mathrm{J}}_{i}\right)=\bar{\delta}^{\mu_{L}-i}(\bar{\nu}, \overline{\mathrm{J}})$ for $0 \leq i \leq \mu_{L}$, define $\bar{c}_{i}=\overline{\operatorname{rk}}\left(\bar{\nu}_{i}, \overline{\mathrm{J}}_{i}\right)$ and 
denote the length of the string in $\left(\bar{\nu}_{i}, \bar{J}_{i}\right)^{(k)}$ selected by $\bar{\delta}$ by $\bar{\ell}_{i}^{(k)}$. Hence to prove that the bijection $\bar{\phi}_{R^{\wedge}}$ maps the image of $\imath^{\wedge}$ onto the image of $\jmath^{\wedge}$ one needs to show that

\section{Claim.}

For $(\nu, J) \in \operatorname{RC}\left(\lambda^{t} ; R^{\wedge t}\right), \ell_{\mu_{L}}^{(k)}=\mu_{L}$ for $0 \leq k<\eta_{L}$ if and only if $\bar{c}_{i}<c_{i}$ for $1 \leq i \leq \mu_{L}$.

We begin by showing that $\bar{c}_{i}<c_{i}$ if $\ell_{\mu_{L}}^{(k)}=\mu_{L}$ for $0 \leq k<\eta_{L}$. First of all notice that

$$
\ell_{i}^{(k)}=i \quad \text { for } 0 \leq k<\eta_{L} \text { and } \quad \bar{\ell}_{i}^{(k)}=i \quad \text { for } 0 \leq k<\eta_{L}-1 .
$$

For $i=\mu_{L}$ this equation holds since both $(\nu, J)=\left(\nu_{\mu_{L}}, J_{\mu_{L}}\right)$ and $(\bar{\nu}, \bar{J})=$ $\left(\bar{\nu}_{\mu_{L}}, \bar{J}_{\mu_{L}}\right)$ are in the image of $\jmath^{\wedge}$. Now assume (4.4) to be true at $i$. This means that there are singular strings of length $i$ in the first $\eta_{L}-1\left(\eta_{L}-2\right)$ partitions of $\nu_{i}\left(\bar{\nu}_{i}\right)$. Hence by construction these turn into singular strings of length $i-1$. Since by definition $\ell_{i-1}^{(0)}=\bar{\ell}_{i-1}^{(0)}=i-1$ this implies (4.4) at $i-1$. We claim that for $k \geq 1$ and $0 \leq i \leq \mu_{L}$

$$
P_{n}^{(k)}\left(\bar{\nu}_{i}\right) \geq J_{n}^{(k)}\left(\bar{\nu}_{i}, \bar{J}_{i}\right)+\sum_{m=1}^{i} \chi\left(\ell_{m}^{(k)} \leq n<\ell_{m}^{(k+1)}\right)
$$

where $J_{n}^{(k)}(\nu, J)$ is the maximal label occurring in the strings of length $n$ in $(\nu, J)^{(k)}$ and $J_{n}^{(k)}(\nu, J)=0$ if there is no string of length $n$ in $\nu^{(k)}$. Equations (4.4) and (4.5) imply

$$
\ell_{i}^{(k+1)} \leq \bar{\ell}_{i}^{(k)} \quad \text { for all } k \geq 0 .
$$

This can be shown by induction on $k$. The initial condition follows immediately from (4.4). By construction $\bar{\ell}_{i}^{(k-1)} \leq \bar{\ell}_{i}^{(k)}$ which becomes by induction hypothesis $\ell_{i}^{(k)} \leq \bar{\ell}_{i}^{(k)}$. Inequality (4.5) implies that there are no singular strings of length $n$ in $\left(\bar{\nu}_{i}, \bar{J}_{i}\right)^{(k)}$ for $\ell_{i}^{(\bar{k})} \leq n<\ell_{i}^{(k+1)}$ which proves (4.6). The condition (4.6) immediately implies that $\bar{c}_{i}<c_{i}$.

It remains to show (4.5), whose proof proceeds by descending induction on $i$, with base case $i=\mu_{L}$. To establish the base case it is shown by induction on $j\left(0 \leq j \leq \mu_{L}\right)$ that

$$
P_{n}^{(k)}\left(\nu_{j}\right) \geq J_{n}^{(k)}\left(\nu_{j}, J_{j}\right)+\sum_{m=j+1}^{\mu_{L}} \chi\left(\ell_{m}^{(k)} \leq n<\ell_{m}^{(k+1)}\right) \quad \text { for all } k \geq 1 .
$$

Since $(\nu, J)=\left(\nu_{\mu_{L}}, J_{\mu_{L}}\right)$ is an admissible rigged configuration, $P_{n}^{(k)}(\nu) \geq$ $J_{n}^{(k)}(\nu, J)$ which implies (4.7) at $j=\mu_{L}$. Now we assume (4.7) to be true at $j+1$ and show its validity at $j$. Since by Proposition 3.12 all $\left(\nu_{j}, J_{j}\right)$ are admissible, $P_{n}^{(k)}\left(\nu_{j}\right) \geq J_{n}^{(k)}\left(\nu_{j}, J_{j}\right)$. This settles (4.7) for $n<\ell_{j+1}^{(k)}$ because in this case the sum over $m$ vanishes since by construction

$$
\ell_{1}^{(k)}<\ell_{2}^{(k)}<\cdots<\ell_{\mu_{L}}^{(k)}
$$


Let us now consider the case $n \geq \ell_{j+1}^{(k)}$. The only string that changes in the transformation $\left(\nu_{j+1}, J_{j+1}\right)^{(k)} \rightarrow\left(\nu_{j}, J_{j}\right)^{(k)}$ is one singular string of length $\ell_{j+1}^{(k)}$. The riggings of all other strings remain unchanged. In particular, $J_{n}^{(k)}\left(\nu_{j}, J_{j}\right)=J_{n}^{(k)}\left(\nu_{j+1}, J_{j+1}\right)$ for $n>\ell_{j+1}^{(k)}$ and $J_{n}^{(k)}\left(\nu_{j}, J_{j}\right) \leq$ $J_{n}^{(k)}\left(\nu_{j+1}, J_{j+1}\right)$ for $n=\ell_{j+1}^{(k)}$. Hence for $n \geq \ell_{j+1}^{(k)}$ we find

$$
\begin{aligned}
P_{n}^{(k)}\left(\nu_{j}\right) & =P_{n}^{(k)}\left(\nu_{j+1}\right)+\chi\left(\ell_{j+1}^{(k)} \leq n<\ell_{j+1}^{(k+1)}\right) \\
& \geq J_{n}^{(k)}\left(\nu_{j+1}, J_{j+1}\right)+\sum_{m=j+1}^{\mu_{L}} \chi\left(\ell_{m}^{(k)} \leq n<\ell_{m}^{(k+1)}\right) \\
& \geq J_{n}^{(k)}\left(\nu_{j}, J_{j}\right)+\sum_{m=j+1}^{\mu_{L}} \chi\left(\ell_{m}^{(k)} \leq n<\ell_{m}^{(k+1)}\right)
\end{aligned}
$$

where the first line follows from $(3.9)$ and $n \geq \ell_{j+1}^{(k)}$, and in the second line the induction hypothesis is used. This concludes the inductive proof of (4.7).

Since by definition $(\bar{\nu}, \bar{J})=\jmath^{\wedge}\left(\nu_{0}, J_{0}\right)$ and $\jmath^{\wedge}$ preserves the vacancy numbers by Lemma 3.9, (4.7) at $j=0$ implies (4.5) at $i=\mu_{L}$. Now assume that (4.5) holds at $i$. Because of the admissibility of $\left(\bar{\nu}_{i-1}, \bar{J}_{i-1}\right)$ and (4.8) at $k+1$, 4.5) holds at $i-1$ for $n \geq \ell_{i-1}^{(k+1)}$. Hence assume $n<\ell_{i-1}^{(k+1)}$. Since $\ell_{i-1}^{(k+1)}<\ell_{i}^{(k+1)} \leq \bar{\ell}_{i}^{(k)}$ by (4.8) and (4.6), in particular $n \leq \bar{\ell}_{i}^{(k)}-2$. Hence we find for $n<\ell_{i-1}^{(k+1)}$

$$
\begin{aligned}
P_{n}^{(k)}\left(\bar{\nu}_{i-1}\right) & =P_{n}^{(k)}\left(\bar{\nu}_{i}\right)-\chi\left(\bar{\ell}_{i}^{(k-1)} \leq n<\bar{\ell}_{i}^{(k)}\right) \\
& \geq J_{n}^{(k)}\left(\bar{\nu}_{i}, \overline{\mathrm{J}}_{i}\right)+\sum_{m=1}^{i-1} \chi\left(\ell_{m}^{(k)} \leq n<\ell_{m}^{(k+1)}\right)
\end{aligned}
$$

where the first line follows from (3.9) and $n \leq \bar{\ell}_{i}^{(k)}-2$, and in the second line the induction hypothesis and (4.6) are used. Since $J_{n}^{(k)}\left(\bar{\nu}_{i-1}, \bar{J}_{i-1}\right)=$ $J_{n}^{(k)}\left(\bar{\nu}_{i}, \bar{J}_{i}\right)$ for $n \leq \bar{\ell}_{i}^{(k)}-2$, this yields (4.5) at $i-1$. This concludes the proof of (4.5) and also that of the forward direction of the claim.

Next we prove the reverse direction of the claim. More precisely, we show that

$$
\ell_{i}^{(k)}=i \quad \text { for } 0 \leq k<\eta_{L} \text { and } 1 \leq i \leq \mu_{L}
$$

if $\bar{c}_{i}<c_{i}$. Since $(\bar{\nu}, \bar{J})=\left(\bar{\nu}_{\mu_{L}}, \bar{J}_{\mu_{L}}\right)$ is in the image of $\jmath^{\wedge}$ it follows by construction that

$$
\bar{\ell}_{i}^{(k)}=i \quad \text { for } 0 \leq k<\eta_{L}-1 \text { and } 1 \leq i \leq \mu_{L} .
$$

We claim that for $k \geq 1$ and $1 \leq i \leq \mu_{L}+1$

$$
P_{n}^{(k)}\left(\nu_{i-1}\right) \geq J_{n}^{(k)}\left(\nu_{i-1}, J_{i-1}\right)+\sum_{m=i}^{\mu_{L}} \chi\left(\bar{\ell}_{m}^{(k-1)} \leq n<\bar{\ell}_{m}^{(k)}\right) .
$$


The condition $\bar{c}_{i}<c_{i}$ and (4.11) imply by induction on $k$ that

$$
\ell_{i}^{(k)} \leq \bar{\ell}_{i}^{(k-1)} \quad \text { for all } 1 \leq i \leq \mu_{L} \text { and } k \geq 1
$$

Before proving this notice the following. Let $\bar{\delta}^{-1}$ be the inverse algorithm of $\bar{\delta}$ as introduced in the proof of Proposition 3.12 and let $s_{i}^{(k)}$ be the length of the singular string in $\left(\nu_{i-1}, J_{i-1}\right)^{(k)}$ selected by $\bar{\delta}^{-1}$. Note that $\bar{\delta}^{-1} \circ \bar{\delta}\left(\nu_{i}, J_{i}\right)=$ $\left(\nu_{i}, J_{i}\right)$ and $s_{i}^{(k)}=\ell_{i}^{(k)}-1$. If $\left(\nu_{i-1}, J_{i-1}\right)^{(k)}$ does not contain singular strings of length $n$ for $a \leq n<b$ and $s_{i}^{(k)}<b$ then by construction $s_{i}^{(k)}<a$ and by $s_{i}^{(k)}=\ell_{i}^{(k)}-1$ also $\ell_{i}^{(k)} \leq a$. Now we prove (4.12) by induction on $k$. For $k>\bar{c}_{i}$ equation (4.12) is true because in this case $\bar{\ell}_{i}^{(k-1)}=\infty$. Now consider $k=\bar{c}_{i}$. Then (4.11) implies that there are no singular strings of length $n \geq \bar{\ell}_{i}^{\left(\bar{c}_{i}-1\right)}$ in $\left(\nu_{i-1}, J_{i-1}\right)^{\left(\bar{c}_{i}\right)}$ since $\bar{\ell}_{i}^{\left(\bar{c}_{i}\right)}=\infty$. Since $\bar{c}_{i}<c_{i}$ the variable $\ell_{i}^{\left(\bar{c}_{i}\right)}$ is finite. This implies that $\ell_{i}^{\left(\bar{c}_{i}\right)} \leq \bar{\ell}_{i}^{\left(\bar{c}_{i}-1\right)}$ which is (4.12) for $k=\bar{c}_{i}$. Now assume 4.12) to be true at $k+1$. By construction $\ell_{i}^{(k)} \leq \ell_{i}^{(k+1)}$ which implies by induction hypothesis that $\ell_{i}^{(k)} \leq \bar{\ell}_{i}^{(k)}$. Because of 4.11) there is no singular string of length $n$ in $\left(\nu_{i-1}, J_{i-1}\right)^{(k)}$ with $\bar{\ell}_{i}^{(k-1)} \leq n<\bar{\ell}_{i}^{(k)}$. Hence $\ell_{i}^{(k)}$ has to obey condition (4.12). Together with $\ell_{i}^{(0)}=i$, 4.12) and (4.10) immediately imply (4.9).

We are left to prove (4.11). Using $\bar{\delta}^{-1}$ one proves in an analogous fashion to 4.7 ) that

$$
P_{n}^{(k)}\left(\bar{\nu}_{j}\right) \geq J_{n}^{(k)}\left(\bar{\nu}_{j}, \overline{\mathrm{J}}_{j}\right)+\sum_{m=1}^{j} \chi\left(\bar{\ell}_{m}^{(k-1)} \leq n<\bar{\ell}_{m}^{(k)}\right)
$$

for all $k \geq 1$ and $0 \leq j \leq \mu_{L}$. Since $\left(\bar{\nu}_{\mu_{L}}, \overline{\mathrm{J}}_{\mu_{L}}\right)=(\bar{\nu}, \overline{\mathrm{J}})=\jmath^{\wedge}\left(\nu_{0}, J_{0}\right)$ and $\jmath^{\wedge}$ preserves the vacancy numbers by Lemma 3.9, this inequality at $j=\mu_{L}$ implies (4.11) at $i=1$. Now assume (4.11) to be true at $i-1$. Using (3.9) and $\ell_{i-1}^{(k)} \leq \bar{\ell}_{i-1}^{(k-1)}$ equation (4.11) at $i$ follows by similar arguments to those used to prove (4.5).

4.2. Direct algorithm for the bijection. Here we state the original algorithm for the bijection $\bar{\phi}_{R}$ between Littlewood-Richardson tableaux and rigged configurations as given in [11]. It combines points 1 and 2 of the Definition-Proposition 4.1 which has the advantage that it is computationally simpler. For the proofs the formulation of Definition-Proposition 4.1 is however more convenient.

Let $T \in \operatorname{CLR}(\lambda ; R)$ be an LR tableaux for a partition $\lambda$ and a sequence of rectangles $R=\left(R_{1}, \ldots, R_{L}\right)$. Set $N=|\lambda|$ and $B_{j}=\left[\left|R_{1}\right|+\cdots+\left|R_{j-1}\right|+\right.$ $\left.1,\left|R_{1}\right|+\cdots+\left|R_{j}\right|\right]$. To obtain $(\nu, J)=\bar{\phi}_{R}(T)$ one recursively constructs a rigged configuration $(\nu, J)_{(x)}$ for each letter $1 \leq x \leq N$ occurring in $T$. Set $(\nu, J)_{(0)}=\emptyset$. Suppose that $x \in B_{j}$, and denote the column index of $x$ in $T$ by $c$ and the column index of $x$ in $\mathrm{ZC}_{j}$ by $c^{\prime}$. Define the numbers $s^{(k)}$ 


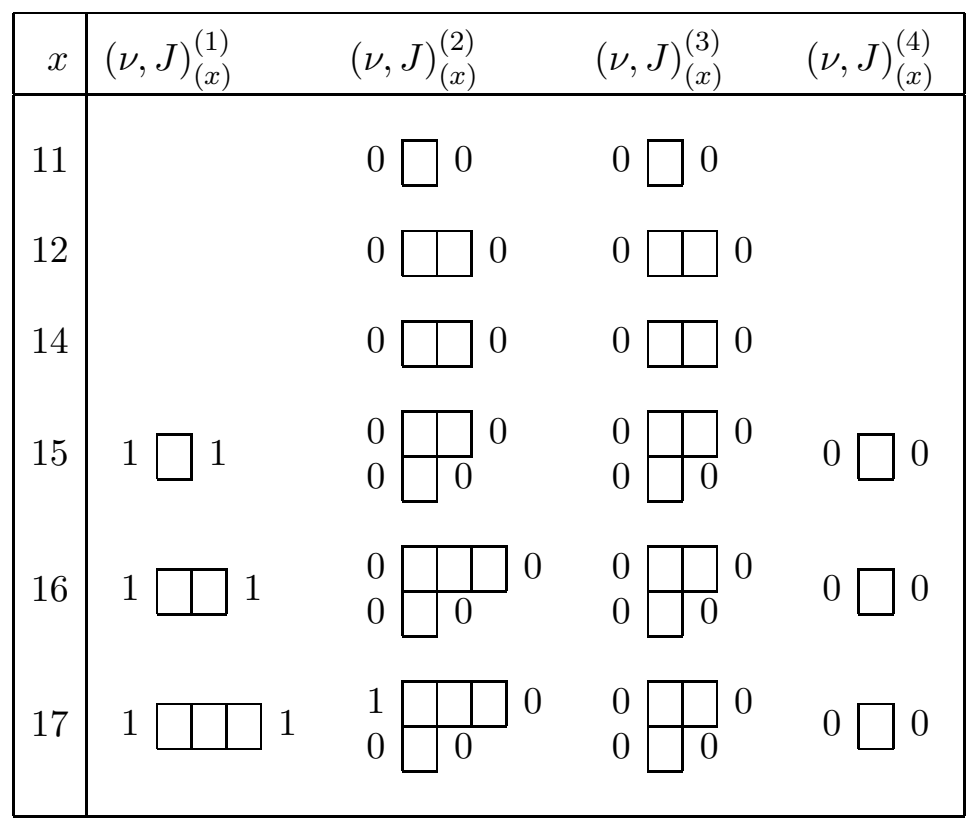

TABLE 2. Example for the bijection algorithm (see Example 4.2)

for $c^{\prime} \leq k<c$ as follows. Let $s^{(c-1)}$ be the length of the longest singular string in $(\nu, J)_{(x-1)}^{(c-1)}$. Now select inductively a singular string in $(\nu, J)_{(x-1)}^{(k)}$ for $k=c-2, c-3, \ldots, c^{\prime}$ whose length $s^{(k)}$ is maximal such that $s^{(k)} \leq s^{(k+1)}$; if no such string exists set $s^{(k)}=0$. Then $(\nu, J)_{(x)}$ is obtained from $(\nu, J)_{(x-1)}$ by adding one box to the selected strings with labels such that they remain singular, leaving all other strings unchanged. Then the image of $T$ under $\bar{\phi}_{R}$ is given by $(\nu, J)=(\nu, J)_{(N)}$.

For the above algorithm it is necessary to be able to compute the vacancy numbers of an intermediate configuration $\nu_{(x)}$. Suppose $x$ occurs in $\mathrm{ZC}_{j}$ in column $c^{\prime}$. In general $R_{(x)}=\left(R_{1}, \ldots, R_{j-1}\right.$, shape $\left.\left(\left.\mathrm{ZC}_{j}\right|_{[1, x]}\right)\right)$ is not a sequence of rectangles. If shape $\left(\left.\mathrm{ZC}_{j}\right|_{[1, x]}\right)$ is not a rectangle one splits it into two rectangles, one of width $c^{\prime}$ and one of width $c^{\prime}-1$. The vacancy numbers are calculated with respect to this new sequence of rectangles.

Example 4.2. The non-trivial steps of the above algorithm applied to the third tableau of Example 2.8 are given in Table 2. A rigged partition is represented by its Ferrers diagram where to the right of each part the corresponding rigging is indicated. The vacancy numbers are given to the left of each part. For example $R_{(12)}=((3,3),(2,2,1,1))$ so that the vacancy numbers of $(\nu, J)_{(12)}$ are calculated with respect to the sequence of rectangles $((3,3),(2,2),(1,1))$.

It is relatively straightforward to see that the above described algorithm is indeed an algorithm for the bijection of Definition-Proposition 4.1. By 
induction it suffices to study the effect of the last $\mu_{L}$ letters of the LR tableaux $T$, that is $N-\mu_{L}<x \leq N$. Recall that $\jmath^{\wedge}$, which corresponds to splitting off the last column of $R_{L}$, adds a singular string of length $\mu_{L}$ to each of the first $\eta_{L}-1$ rigged partitions and leaves the vacancy numbers invariant. By construction, these extra singular strings are removed by $\bar{\delta}^{\mu_{L}}$. This has the same effect as restricting the removal/addition of boxes to the partitions $\nu_{(x)}^{(k)}$ with $\eta_{L}=c^{\prime} \leq k$ as is the case for the algorithm of this section. One may also show that the vacancy numbers of the intermediate rigged partitions obtained from the the algorithm and the recursive definition of DefinitionProposition 4.1 are the same for $\nu_{(x)}^{(k)}$ with $k \neq \eta_{L}-1$. For $\nu_{(x)}^{\left(\eta_{L}-1\right)}$ the vacancy numbers from the algorithm cannot be smaller than the corresponding ones coming from Definition-Proposition 4.1, and are the same when $R_{(x)}$ is a sequence of rectangles. This difference for non-rectangular $R_{(x)}$ is harmless since no strings in $\nu_{(x)}^{\left(\eta_{L}-1\right)}$ are changed by the algorithm.

\section{Evacuation Theorem}

In this Section we prove the Evacuation Theorem 5.6 which relates the evacuation of LR tableaux to the complementation of quantum numbers on rigged configurations. The proof requires intertwining relations of $\bar{\phi}_{R}$ with $R \rightarrow R^{\vee}$ and $R \rightarrow \widetilde{R}$ which are derived in Section 5.1.

\subsection{Intertwining of $\bar{\phi}_{R}$ with $R \rightarrow R^{\vee}$ and $R \rightarrow \widetilde{R}$.}

Lemma 5.1. $\jmath^{\wedge} \circ \jmath^{\vee}=\jmath^{\vee} \circ \jmath^{\wedge}$.

Proof. If $R$ consists of more than one rectangle or $R$ is a single rectangle with more than two columns, the commutativity of $\jmath^{\wedge}$ and $\jmath^{\vee}$ is obvious. If $R$ is a single column then both $\jmath^{\wedge}$ and $\jmath^{\vee}$ are the identity and obviously commute. So it may be assumed that $R$ is a single rectangle with exactly two columns. Then $R^{\wedge}=R^{\vee}=\left(\left(1^{\mu_{1}}\right),\left(1^{\mu_{1}}\right)\right)$. This means that the outer function in both $\jmath^{\wedge} \circ \jmath^{\vee}$ and $\jmath^{\vee} \circ \jmath^{\wedge}$ acts as the identity. So $R^{\wedge \vee}=R^{\wedge}, R^{\vee \wedge}=R^{\vee}$, and for all $(\nu, J) \in \operatorname{RC}\left(\lambda^{t} ; R^{t}\right), \jmath^{\wedge}\left(\jmath^{\vee}(\nu, J)\right)=\jmath^{\vee}(\nu, J)$ and $\jmath^{\vee}\left(\jmath^{\wedge}(\nu, J)\right)=\jmath^{\wedge}(\nu, J)$. So it must be shown that $\jmath^{\vee}(\nu, J)=g^{\wedge}(\nu, J)$. Since $\jmath^{\wedge}$ (resp. $\jmath^{\vee}$ ) adds a string of length $\mu_{1}$ with singular (resp. zero) label to the first rigged partition of $(\nu, J)$, it must be shown that $P_{\mu_{1}}^{(1)}(\nu)=0$ for all $(\nu, J) \in \mathrm{RC}\left(\lambda^{t} ; R^{t}\right)$. It may be assumed that $\lambda=R_{1}$ for otherwise $\operatorname{RC}\left(\lambda^{t} ; R^{t}\right)$ is empty. Then $\operatorname{RC}\left(\lambda^{t} ; R^{t}\right)$ is the singleton set consisting of the empty rigged configuration $(\emptyset, \emptyset)$. One computes the vacancy number $P_{\mu_{1}}^{(1)}(\emptyset)=0$.

Lemma 5.2. Suppose the last rectangle of $R$ is a single column. Then $\bar{\delta} \circ \jmath^{\vee}=\jmath^{\vee} \circ \bar{\delta}$.

Proof. Let $\bar{\ell}^{(k)}$ be the lengths of strings selected by $\bar{\delta}$ acting on $j^{\vee}(\nu, J)=$ $\left(\nu^{\vee}, J^{\vee}\right)$. To prove the lemma it suffices to show that $\bar{\ell}^{(k)}=\bar{\ell}^{(k)}$ for all $k \geq 1$. By Lemma 3.10, $P_{n}^{(k)}(\nu)=P_{n}^{(k)}\left(\nu^{\vee}\right)$ for all $k, n \geq 1$. Since $\left(\nu^{\vee}, J^{\vee}\right)^{(k)}$ 
is obtained from $(\nu, J)^{(k)}$ by adding the string $\left(\mu_{1}, 0\right)$ for $1 \leq k \leq \eta_{1}-1$, it is clear that $\bar{\ell}^{(k)} \leq \bar{\ell}^{(k)}$ for all $k$. Let $k$ be minimal such that $\bar{\ell}^{(k)}<$ $\bar{\ell}^{(k)}$. Then the string $\left(\mu_{1}, 0\right)$ was selected in $\left(\nu^{\vee}, J^{\vee}\right)^{(k)}$, so that $\bar{\ell}^{(k)}=\mu_{1}$ and $P_{\mu_{1}}^{(k)}\left(\nu^{\vee}\right)=P_{\mu_{1}}^{(k)}(\nu)=0$. Now $\bar{\ell}^{(k-1)}={\overline{\ell^{\vee}}}^{(k-1)} \leq{\overline{\ell^{\vee}}}^{(k)}=\mu_{1}$ and $\mu_{1}=\bar{\ell}^{(k)}<\bar{\ell}^{(k)}$. By (3.9) we have $P_{\mu_{1}}^{(k)}(\bar{\nu})=P_{\mu_{1}}^{(k)}(\nu)-\chi\left(\bar{\ell}^{(k-1)} \leq \mu_{1}<\right.$ $\left.\bar{\ell}^{(k)}\right)+\chi\left(\bar{\ell}^{(k)} \leq \mu_{1}<\bar{\ell}^{(k+1)}\right)=-1$, which is a contradiction. Thus there is no such $k$.

For future use let us formalize a general nonsense lemma.

Lemma 5.3. Suppose we have a diagram of the following kind:

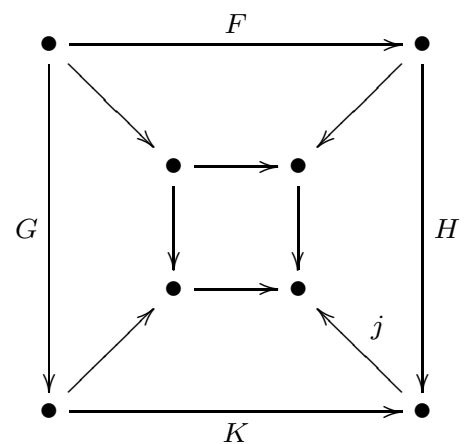

Viewing this diagram as a cube with front face given by the large square, suppose the square diagrams given by all the faces of the cube except the front, commute. Assume also that the map $j$ is injective. Then the front face must also commute.

Proof. The commuting faces yield the equality $j \circ K \circ G=j \circ H \circ F$. Since $j$ is injective this implies $K \circ G=H \circ F$ as desired.

The following Lemma gives the intertwining of $\bar{\phi}_{R}$ with $R \rightarrow R^{\vee}$.

Lemma 5.4. The following diagram commutes:

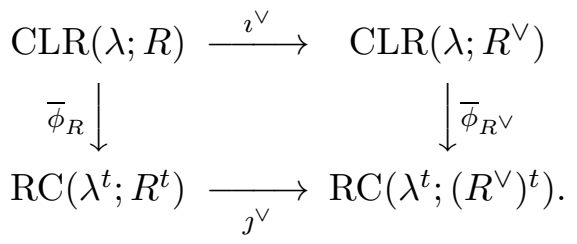

Proof. The proof proceeds by the induction that defines the bijection $\bar{\phi}$. 
Suppose the last rectangle of $R$ has more than one column; this subsumes the base case $R=\left(R_{1}\right)$. Clearly $R^{\vee \wedge}=R^{\wedge \vee}$. Consider the diagram

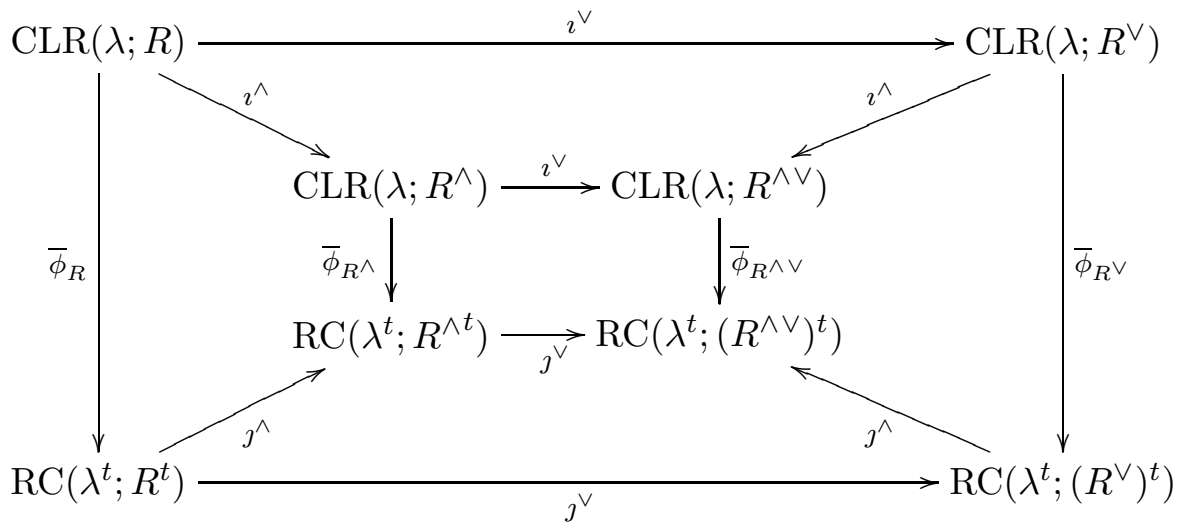

We wish to show the front face commutes. By Lemma 5.3 and the injectivity of $g^{\wedge}$, it is enough to show that all the other faces commute. The back face is assumed to commute by induction, the left and right faces commute by the definition of the bijections $\bar{\phi}$ (see (4.3)), the commutation of the top face is obvious, and the commutativity of the bottom face follows from Lemma 5.1.

For the remaining case suppose the last rectangle of $R$ is a single column. Clearly $\bar{R}^{\vee}=\overline{R^{\vee}}$. Consider the diagram

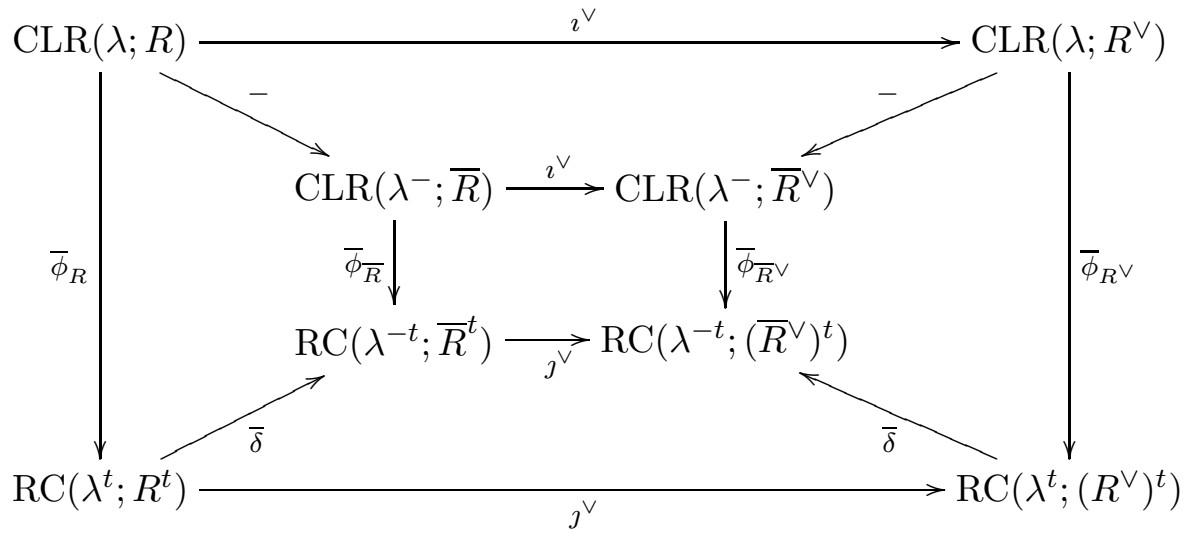

$\bar{\delta}$ is injective, so again by Lemma 5.3 it suffices to check that all faces but the front, commute. The back face commutes by induction. The left and right faces commute by the definition of $\bar{\phi}$. The top face obviously commutes. The bottom face commutes by Lemma 5.2.

The intertwining relation of $\bar{\phi}_{R}$ with $R \rightarrow \widetilde{R}$ is stated in the next Lemma. 
Lemma 5.5. Suppose the first rectangle of $R$ is a single column. Then the following diagram commutes:

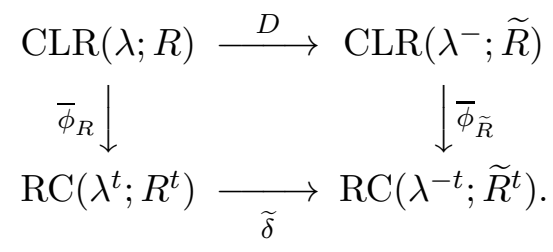

Proof. Suppose that the last rectangle of $R$ is also a single column; this case subsumes the base case that $R=\left(R_{1}\right)$ is a single column. Clearly $\widetilde{\bar{R}}=\widetilde{\widetilde{R}}$.

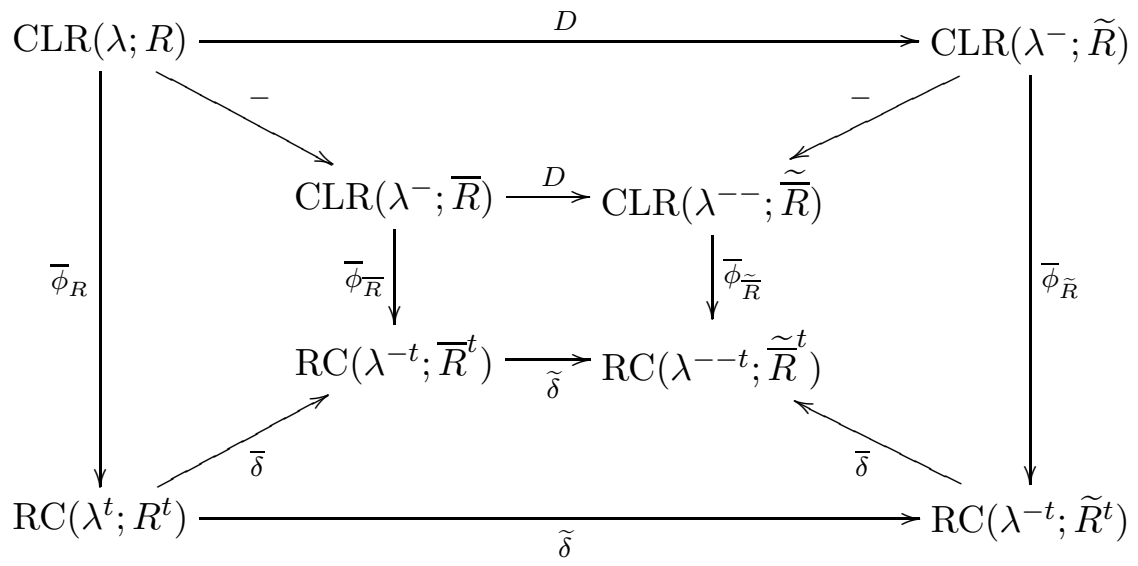

In this diagram there is a map

$$
\bar{\phi}_{\bar{R}}: \operatorname{CLR}\left(\lambda^{--} ; \widetilde{\bar{R}}\right) \rightarrow \operatorname{RC}\left(\lambda^{--t} ; \widetilde{\bar{R}}^{t}\right)
$$

This is to be understood in the most obvious way, namely, that given a chain of partitions $\mathcal{C}=(\sigma \lessdot \rho \lessdot \lambda)$, and $T \in \operatorname{CLR}(\sigma ; \widetilde{\bar{R}})$, then $\bar{\phi}_{\overline{\bar{R}}}\left(T_{\mathcal{C}}\right)=\bar{\phi}_{\bar{R}}(T)_{\mathcal{C}}$, that is, the copy of $T \in \operatorname{CLR}(\sigma ; \widetilde{\bar{R}})$ labeled by $\mathcal{C}$ is mapped to the copy of $\bar{\phi}_{\bar{R}}(T) \in \operatorname{RC}\left(\sigma^{t} ; \widetilde{\bar{R}}^{t}\right)$ indexed by the same chain $\mathcal{C}$.

We use the approach of Lemma 5.3. The back face commutes by induction, the left and right faces by the definition of $\bar{\phi}$, the top face commutes up to the involution $\tau$ on $\operatorname{CLR}\left(\lambda^{--} ; \widetilde{\bar{R}}\right)$ by $(3.4)$, and the bottom face commutes up to the involution $\tau$ on $\operatorname{RC}\left(\lambda^{--t} ; \tau^{t}\right)$ by $(3.17)$. Based on the above commutation up to $\tau$, it still follows that $\bar{\delta} \circ \widetilde{\delta} \circ \bar{\phi}_{R}=\bar{\delta}_{\circ} \circ \bar{\phi}_{\widetilde{R}} \circ D$ as maps into $\mathrm{RC}\left(\lambda^{--t} ; \widetilde{\bar{R}}^{t}\right)$. Moreover $\bar{\delta}$ is injective as a map into the disjoint union $\mathrm{RC}\left(\lambda^{--t} ; \widetilde{\bar{R}}^{t}\right)$ so it follows that the front face commutes. 
In the remaining case, the last rectangle of $R$ has more than one column. Then $\widetilde{R}^{\wedge}=\widetilde{R^{\wedge}}$. We have the following diagram:

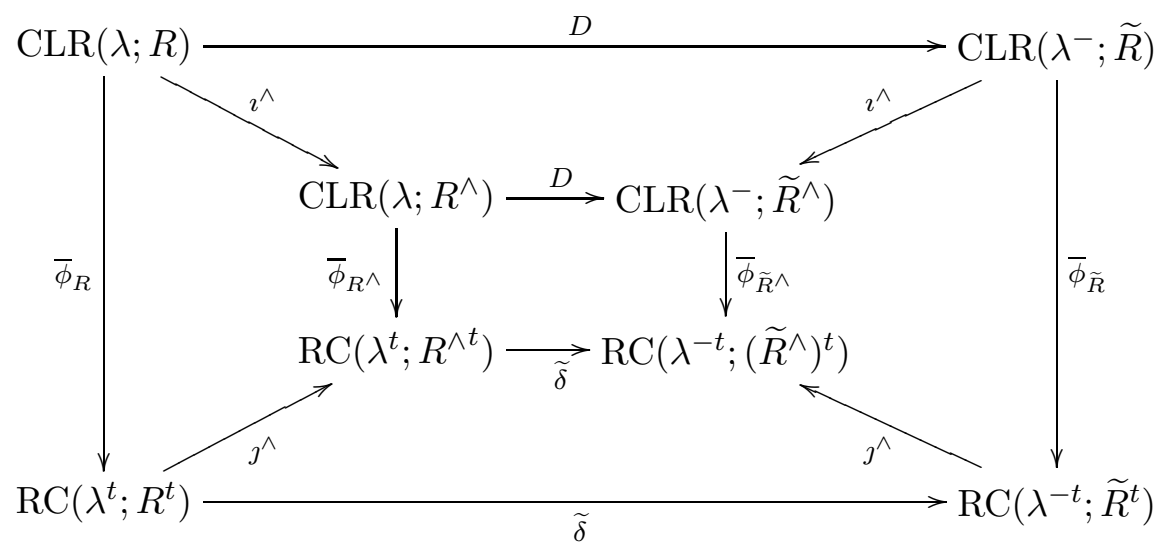

$\jmath^{\wedge}$ is injective, the back face commutes by induction and the left and right faces by the definition of $\bar{\phi}$. The commutation of the top face is obvious. The bottom face commutes by conjugating the result of Lemma 5.2 by $\theta^{\mathrm{ev}}$. By Lemma 5.3 the front face commutes.

\subsection{Proof of the Evacuation Theorem.}

Theorem 5.6 (Evacuation Theorem). The following diagram commutes:

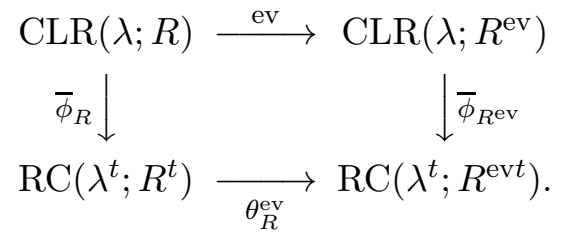

Proof. If $R$ is empty the result holds trivially. Suppose that the last rectangle of $R$ has more than one column. Obviously $R^{\wedge \mathrm{ev}}=\left(R^{\mathrm{ev}}\right)^{\vee}$ and $R^{\vee \mathrm{ev}}=$ $\left(R^{\mathrm{ev}}\right)^{\wedge}$. Consider the diagram:

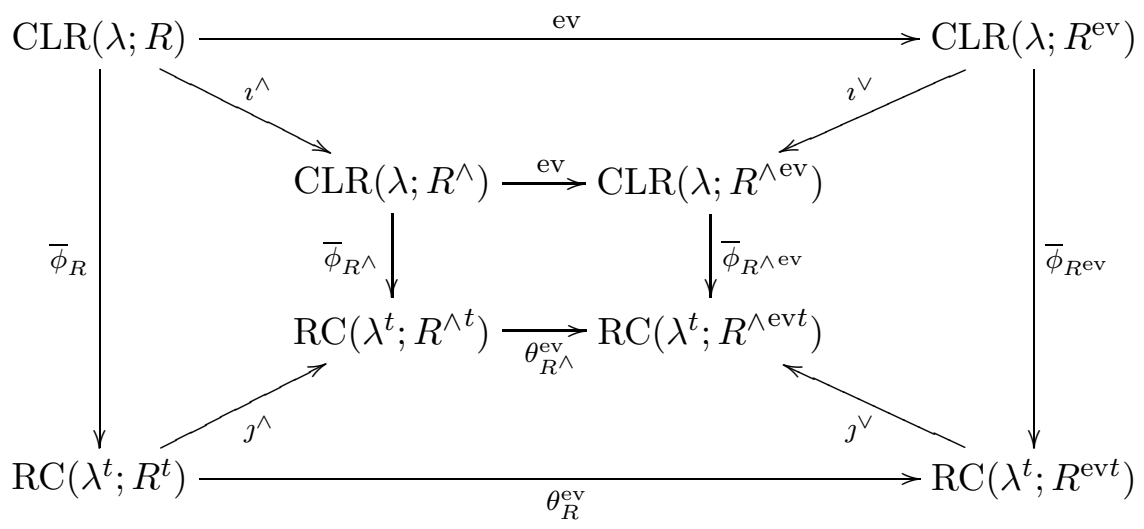


$\jmath^{\vee}$ is injective, the back face commutes by induction, the top and bottom faces are given by the commutative diagrams (3.6) and (3.8), the left face commutes by the definition of $\bar{\phi}_{R}$, and the right face commutes by (5.1) with $R^{\mathrm{ev}}$ in place of $R$. So by Lemma 5.3 the front face commutes.

Suppose that the last rectangle of $R$ is a single column. Obviously $\bar{R}^{\text {ev }}=$ $\widetilde{R^{\mathrm{ev}}}$ and $\widetilde{R}^{\mathrm{ev}}=\overline{R^{\mathrm{ev}}}$. Consider the diagram:

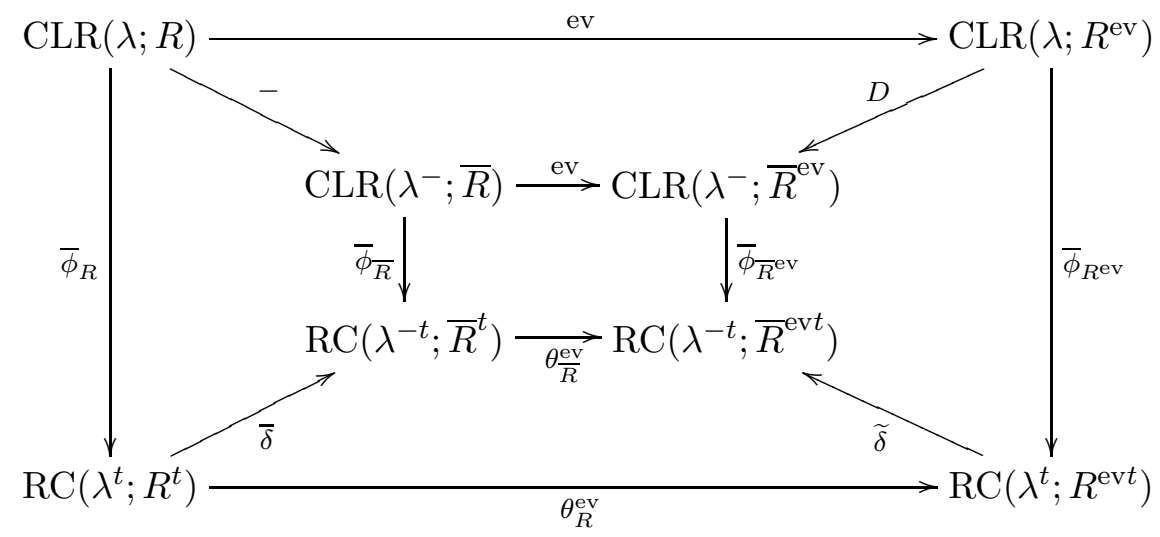

$\widetilde{\delta}$ is injective, the back face commutes by induction, the top face commutes by Proposition 3.7, the bottom face commutes by (3.15) replacing $R$ by $R^{\mathrm{ev}}$ and using that $\theta_{R}^{\text {ev }}$ is an involution, the left face commutes by the definition of $\bar{\phi}_{R}$, and the right face commutes by (5.2) with $R^{\mathrm{ev}}$ in place of $R$. So by Lemma 5.3 the front face commutes.

\section{Another ReCURREnCE For $\bar{\phi}_{R}$}

The bijection $\bar{\phi}_{R}$ is defined by a recurrence that removes columns from the last rectangle. In this section it is shown that $\bar{\phi}_{R}$ may be defined by an analogous recurrence which removes rows from the last rectangle. This recurrence shall be used to prove some properties of the transpose maps on LR tableaux and rigged configurations.

6.1. Splitting off the first or last row. Let $R^{<}$be obtained from $R$ by splitting off the first row from the first rectangle so that the first rectangle in $R^{<}$is $\left(\eta_{1}\right)$. Similarly, let $R^{>}$be obtained from $R$ by splitting off the last row from the last rectangle. Recall the transpose map $\operatorname{tr}_{\mathrm{LR}}$ of Definition 2.7. Note that $\left(R^{t}\right)^{\vee}=R^{<t}$. Define $\imath^{<}: \operatorname{CLR}(\lambda ; R) \rightarrow \operatorname{CLR}\left(\lambda ; R^{<}\right)$as

$$
\imath^{<}:=\operatorname{tr}_{\mathrm{LR}} \circ \imath^{\vee} \circ \operatorname{tr}_{\mathrm{LR}} \text {. }
$$

Note that $R^{>t}=\left(R^{t}\right)^{\wedge}$. Similarly define $\imath^{>}: \operatorname{CLR}(\lambda ; R) \rightarrow \operatorname{CLR}\left(\lambda ; R^{>}\right)$as

$$
\imath^{>}:=\operatorname{tr}_{\mathrm{LR}} \circ \imath^{\wedge} \circ \operatorname{tr}_{\mathrm{LR}} \text {. }
$$

Observing that $R^{>\mathrm{ev}}=R^{\mathrm{ev}<}$, it follows from the definitions of $\imath^{<}$and $\imath^{>}$, (3.6) and the commutativity of $\operatorname{tr}_{\mathrm{LR}}$ and ev that the following diagram 
commutes:

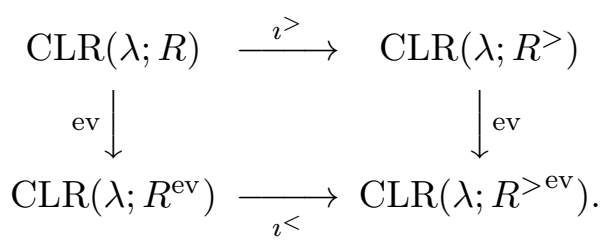

Let $\jmath^{>}: \operatorname{RC}\left(\lambda^{t} ; R^{t}\right) \rightarrow \operatorname{RC}\left(\lambda^{t} ; R^{>t}\right)$ be the inclusion map. Then define $\jmath^{<}$ by the following commutative diagram:

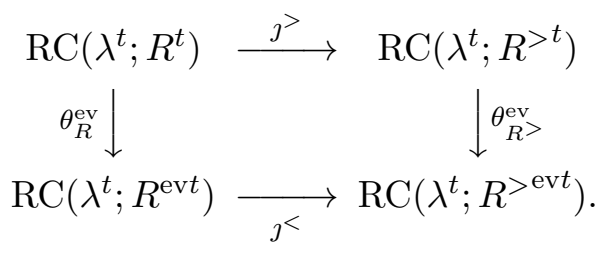

For $(\nu, J) \in \operatorname{RC}\left(\lambda^{t} ; R^{t}\right)$ set $\left(\nu^{>}, J^{>}\right)=\jmath^{>}(\nu, J)$. Note that

$$
P_{n}^{(k)}\left(\nu^{>}\right)=P_{n}^{(k)}(\nu)+\chi\left(k=\eta_{L}\right) \chi\left(1 \leq n<\mu_{L}\right) .
$$

Since $\theta_{R}^{\text {ev }}$ reverses the sequence of rectangles and complements the quantum numbers this implies that $\jmath^{<}(\nu, J)$ is obtained from $(\nu, J)$ by replacing $(n, x) \in(\nu, J)^{\left(\eta_{1}\right)}$ by $(n, x+1)$ for $1 \leq n<\mu_{1}$, and leaving all other riggings invariant. In particular, $\jmath^{<}$preserves the colabels.

Lemma 6.1. The following diagram commutes

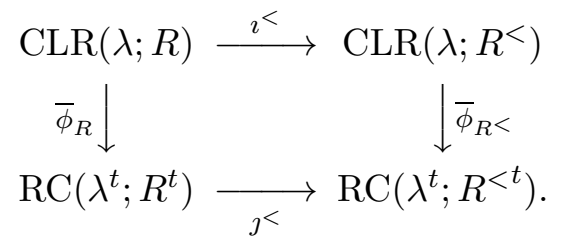

Proof. Suppose first that $L \geq 2$ and that $R_{L}$ has more than one column. Note that $R^{<\wedge}=R^{\wedge<}$.

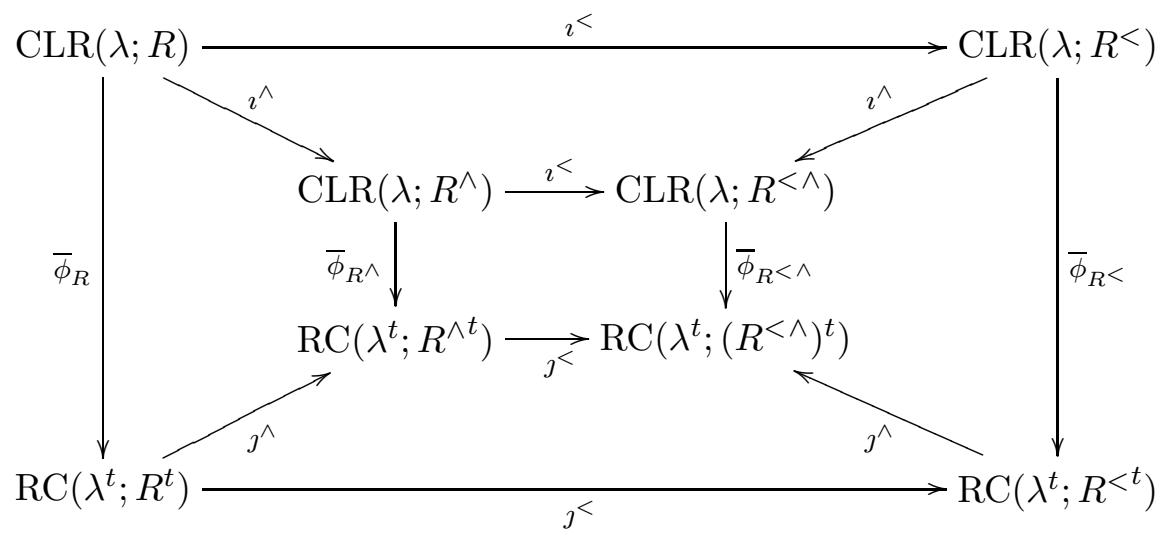


The top face commutes since $\imath^{<}$and $\imath^{\wedge}$ are both relabelings that replace different subalphabets. The bottom face commutes since $\jmath^{\wedge}$ adds singular strings and $\jmath^{<}$preserves colabels and hence preserves singularity of strings. The left and right faces commute by the definition of $\bar{\phi}$, and the back face commutes by induction. Since $y^{\wedge}$ is injective, by Lemma 5.3 the front face commutes as desired.

Suppose $L \geq 2$ and the last rectangle is a single column.

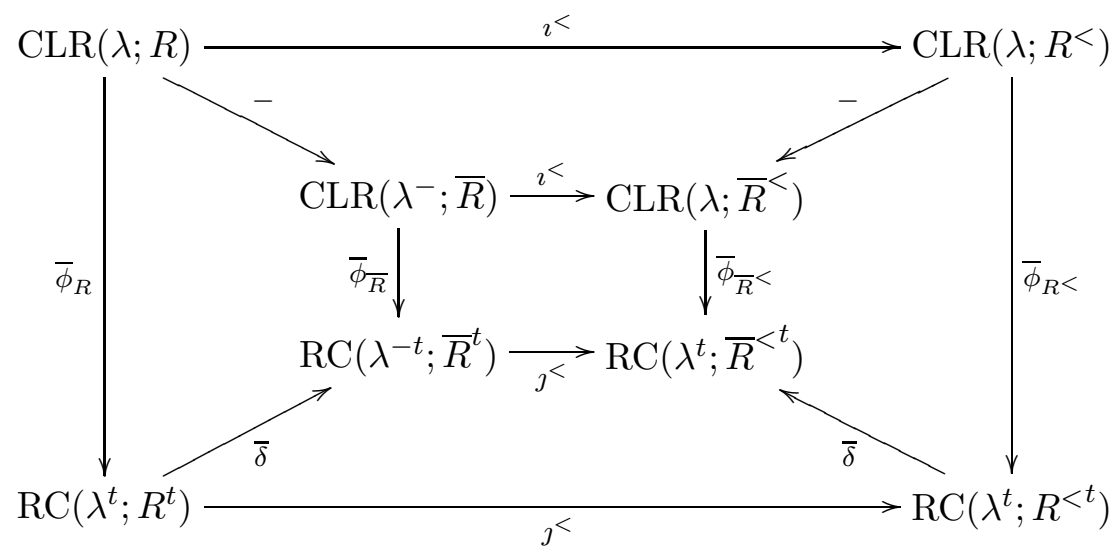

The commutation of the top face is obvious. Since $\jmath^{<}$preserves colabels it is straightforward to verify that the bottom face commutes. The left and right faces commute by the definition of $\bar{\phi}$ and the back face by induction. Since $\bar{\delta}$ is injective by Lemma 5.3 the front face commutes.

The remaining case is $L \leq 1$. It may be assumed that $\operatorname{CLR}(\lambda ; R) \neq$ $\emptyset$ for otherwise there is nothing to show. By [22, Prop. 33] $\operatorname{CLR}(\lambda ; R)$ is a singleton if $R$ has at most two rectangles. But $\imath^{<}: \operatorname{CLR}(\lambda ; R) \rightarrow$ $\operatorname{CLR}\left(\lambda ; R^{<}\right)$is an embedding and $R^{<}$has at most two rectangles so that $\operatorname{CLR}\left(\lambda ; R^{<}\right)$is a singleton. Since $\bar{\phi}_{R}$ and $\bar{\phi}_{R^{<}}$are bijections, $\operatorname{RC}\left(\lambda^{t} ; R^{t}\right)$ and $\operatorname{RC}\left(\lambda^{t} ; R^{<t}\right)$ are also singletons. So the embedding $\jmath^{<}$must send the unique element of $\operatorname{RC}\left(\lambda^{t} ; R^{t}\right)$ to the unique element of $\operatorname{RC}\left(\lambda^{t} ; R^{<t}\right)$ and the required commutation follows.

Lemma 6.2. The following diagram commutes:

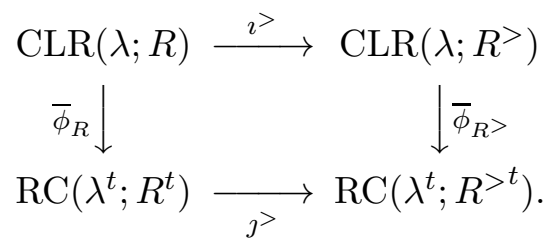


Proof. Consider the diagram

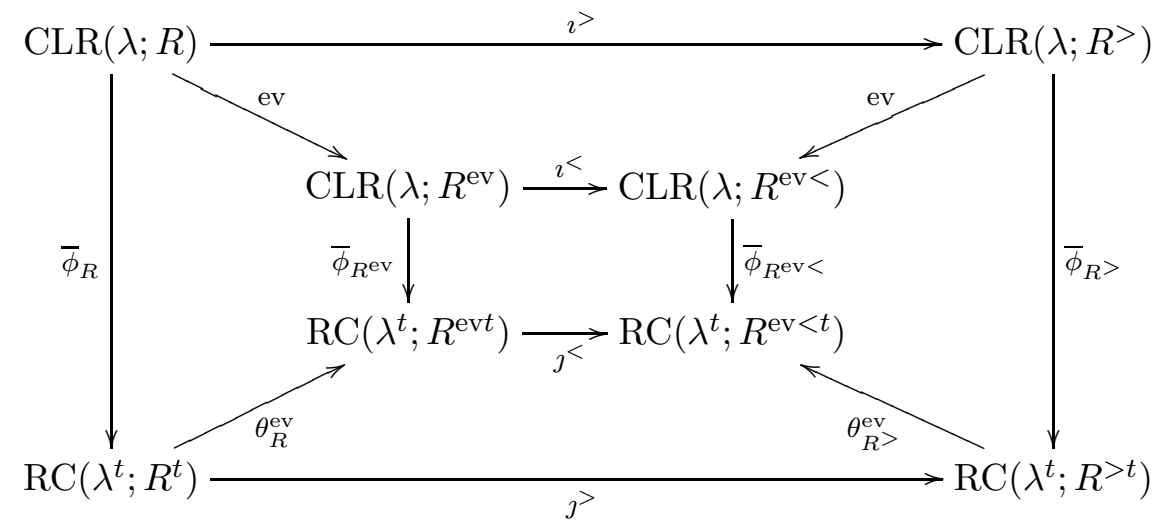

The back face commutes by (6.4) for $R^{\mathrm{ev}}$, the top and bottom faces commute by $(\sqrt[6.2]{ })$ and $(\sqrt[6.3]{)}$, the left and right faces commute by the Evacuation Theorem 5.6 for $R$ and $R^{>}$, and $\theta_{R>}^{\mathrm{ev}}$ is injective. Therefore the front face commutes by Lemma 5.3 .

6.2. Removal of a cell from a single row. Suppose $R_{L}$ is a single row. Then $R^{\wedge}$ is given by splitting off one cell from the end of the row $R_{L}$, and $\overline{R^{\wedge}}$ is obtained from $R$ by removing the cell at the end of $R_{L}$. As usual define $\operatorname{CLR}\left(\lambda^{-} ; \overline{R^{\wedge}}\right):=\bigcup_{\rho \lessdot \lambda} \operatorname{CLR}\left(\rho ; \overline{R^{\wedge}}\right)$ and $\operatorname{RC}\left(\lambda^{-t} ;{\overline{R^{\wedge}}}^{t}\right):=\bigcup_{\rho \lessdot \lambda} \operatorname{RC}\left(\rho^{t} ; \overline{R^{\wedge}}{ }^{t}\right)$. The bijection - : $\mathrm{ST}(\lambda) \rightarrow \mathrm{ST}\left(\lambda^{-}\right)$restricts to an injection

$$
-: \operatorname{CLR}(\lambda ; R) \rightarrow \operatorname{CLR}\left(\lambda^{-} ; \overline{R^{\wedge}}\right) .
$$

Define the map $\partial: \operatorname{RC}\left(\lambda^{t} ; R^{t}\right) \rightarrow \mathrm{RC}\left(\lambda^{-t} ;{\overline{R^{\wedge}}}^{t}\right)$ by the following algorithm. Let $(\nu, J) \in \operatorname{RC}\left(\lambda^{t} ; R^{t}\right)$. Define the integers $\ell^{(0)}=\cdots=\ell^{\left(\eta_{L}-1\right)}=1$. For $k \geq \eta_{L}$, inductively define $\ell^{(k)}$ to be minimal such that $\ell^{(k)} \geq \ell^{(k-1)}$ and there is a singular string of length $\ell^{(k)}$ in $(\nu, J)^{(k)}$. Let $\underline{\mathrm{rk}}(\nu, J)$ be the minimal index such that such a singular string does not exist, and set $\ell^{(k)}=\infty$ for $k \geq \underline{\mathrm{rk}}(\nu, J)$. Then $\partial(\nu, J)$ is obtained from $(\nu, J)$ by shortening each of the selected singular strings in $(\nu, J)^{(k)}$ (for $\eta_{L} \leq k<\underline{\mathrm{rk}}(\nu, J)$ ) by one and keeping them singular, and leaving all other strings unchanged.

Lemma 6.3. Suppose the last rectangle of $R$ is a single row. The following diagram commutes:

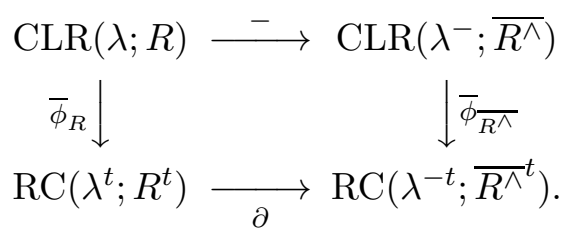


Proof. Consider the diagram

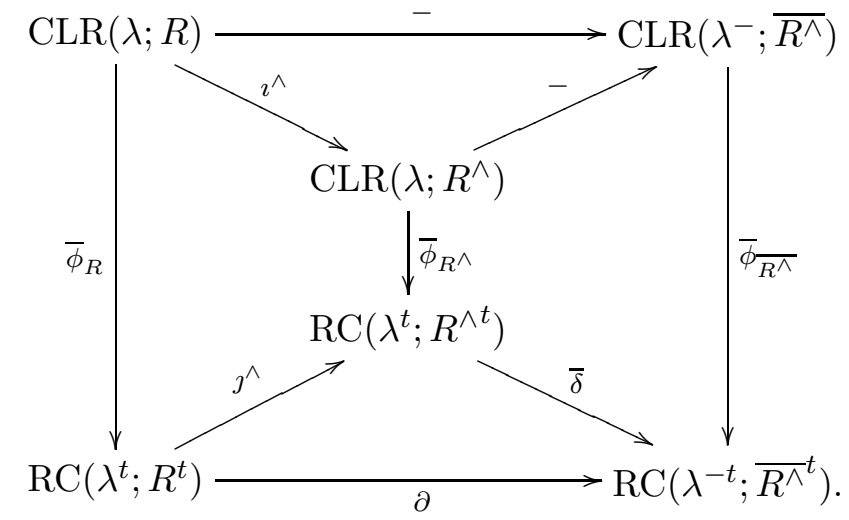

This diagram may be viewed as a prism whose top and bottom are triangles and whose front is the diagram (6.6) which must be proven. The back left and back right faces commute by the definition of $\bar{\phi}$. The top triangle obviously commutes. It suffices to show that the bottom triangle commutes. This is done by computing $\bar{\delta} \circ \jmath^{\wedge}$ explicitly. Let $(\nu, J) \in \operatorname{RC}\left(\lambda^{t} ; R^{t}\right)$. Then $\jmath^{\wedge}(\nu, J)=\left(\nu^{\wedge}, \mathrm{J}^{\wedge}\right)$ is obtained from $(\nu, J)$ by adding a singular string of length 1 to each of the first $\eta_{L}-1$ rigged partitions. Let $\bar{\ell}^{(k)}$ be the lengths of the singular strings chosen by $\bar{\delta}$ acting on $\left(\nu^{\wedge}, \mathrm{J}^{\wedge}\right)$. Since $\bar{\ell}^{(0)}=1$ by definition, the singular strings that were added by $\jmath^{\wedge}$, are selected by $\bar{\delta}$, so $\bar{\ell}^{(k)}=1$ for $1 \leq k \leq \eta_{L}-1$. Then it is obvious that for $k \geq \eta_{L}$ that $\bar{\delta}$ acting on $\left(\nu^{\wedge}, \mathrm{J}^{\wedge}\right)$, selects the same strings that $\partial$ does, acting on $(\nu, J)$. The result is now clear.

6.3. The new recurrence for $\bar{\phi}_{R}$. If the last rectangle of $R$ is a single row, then one may use the commutative diagram (6.6) to express $\bar{\phi}_{R}$ inductively in terms of $\bar{\phi} \overline{R^{\wedge}}$. If the last rectangle of $R$ has more than one row, then one may apply the commutative diagram 6.5 to express $\bar{\phi}_{R}$ in terms of $\bar{\phi}_{R^{>}}$. It will be shown in Section 7 that this recurrence, which also defines $\bar{\phi}_{R}$, is in a sense transpose to the usual definition of the bijection $\bar{\phi}_{R^{t}}$.

\section{TRANSPOSE MAPS}

Recall the LR-transpose bijection $\operatorname{tr}_{\mathrm{LR}}: \operatorname{CLR}(\lambda ; R) \rightarrow \operatorname{CLR}\left(\lambda^{t} ; R^{t}\right)$ of Definition 2.7. An analogous RC-transpose bijection exists for the set of rigged configurations denoted by $\operatorname{tr}_{\mathrm{RC}}: \mathrm{RC}\left(\lambda^{t} ; R^{t}\right) \rightarrow \mathrm{RC}(\lambda ; R)$, which was described in [13, Section 9]. In the following we recall its definition and prove the Transpose Theorem 7.1.

Let $(\nu, J) \in \operatorname{RC}\left(\lambda^{t} ; R^{t}\right)$ and let $\nu$ have the associated matrix $m$ with entries $m_{i j}$ as in [13, (9.2)]

$$
m_{i j}=\alpha_{j}^{(i-1)}-\alpha_{j}^{(i)}
$$


for $i, j \geq 1$, where $\alpha_{j}^{(i)}$ is the size of the $j$-th column of the partition $\nu^{(i)}$, recalling that $\nu^{(0)}$ is defined to be the empty partition. The configuration $\nu^{t}$ in $\left(\nu^{t}, J^{t}\right)=\operatorname{tr}_{\mathrm{RC}}(\nu, J)$ is defined by its associated matrix $m^{t}$ given by

$$
m_{i j}^{t}=-m_{j i}+\chi((i, j) \in \lambda)-\sum_{a=1}^{L} \chi\left((i, j) \in R_{a}\right)
$$

for all $i, j \geq 1$. Here $(i, j) \in \lambda$ means that the cell $(i, j)$ is in the Ferrers diagram of the partition $\lambda$ with $i$ specifying the row and $j$ the column.

For all $k, n \geq 1$, a rigging $J$ of $\nu$ determines a partition $J_{n}^{(k)}$ inside the rectangle of height $m_{n}\left(\nu^{(k)}\right)$ and width $P_{n}^{(k)}(\nu)$ given by the labels of the parts of $\nu^{(k)}$ of size $n$. The partition $J_{k}^{t(n)}$ corresponding to $\left(\nu^{t}, J^{t}\right)=\operatorname{tr}_{\mathrm{RC}}(\nu, J)$ is defined as the transpose of the complementary partition to $J_{n}^{(k)}$ in the rectangle of height $m_{n}\left(\nu^{(k)}\right)$ and width $P_{n}^{(k)}(\nu)$.

By [13, (9.7)] and [13, Lemma 10], it follows that

$$
\begin{aligned}
& \text { if } m_{n}\left(\nu^{(k)}\right)>0 \text { then } P_{n}^{(k)}(\nu)=m_{k}\left(\nu^{t(n)}\right) \\
& \text { if } m_{k}\left(\nu^{t(n)}\right)>0 \text { then } P_{k}^{(n)}\left(\nu^{t}\right)=m_{n}\left(\nu^{(k)}\right) .
\end{aligned}
$$

This is weaker than the assertion [13, (9.10)], which does not seem to follow from only the assumption $m_{n}\left(\nu^{(k)}\right)>0$ or $m_{k}\left(\nu^{t(n)}\right)>0$. Fortunately equation (7.3) still suffices to well-define the map $\operatorname{tr}_{R C}$. To see this, observe that it still follows from [13, Section 9] that $\operatorname{tr}_{\mathrm{RC}}$ is an involution on the level of configurations. For $\operatorname{tr}_{\mathrm{RC}}$ to be well-defined for the riggings, it is enough to show that the rectangle of height $m_{n}\left(\nu^{(k)}\right)$ and width $P_{n}^{(k)}(\nu)$ and the rectangle of height $m_{k}\left(\nu^{t(n)}\right)$ and width $P_{k}^{(n)}\left(\nu^{t}\right)$, are either transposes of each other or are both empty. But this follows from (7.3). Hence $\operatorname{tr}_{\mathrm{RC}}$ is an involution for rigged configurations.

We shall prove [13, Conjecture 16].

Theorem 7.1 (Transpose Theorem). The following diagram commutes:

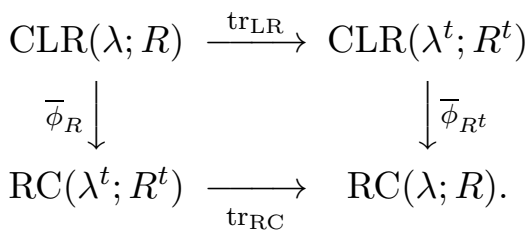

Theorem 7.1 follows again from Lemma 5.3 by the usual arguments and requires the following two results. Note that $R^{t>t}=R^{\wedge}$.

Lemma 7.2. The following diagram commutes:

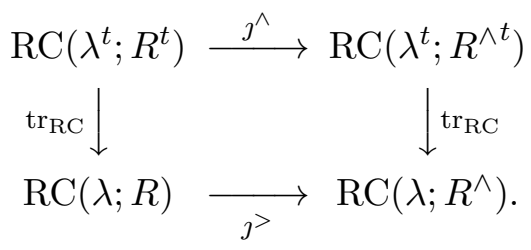


Proof. Let $(\nu, J) \in \mathrm{RC}\left(\lambda^{t} ; R^{t}\right)$ and set $\left(\nu^{\wedge}, \mathrm{J}^{\wedge}\right)=\jmath^{\wedge}(\nu, J)$. Recall that $\left(\nu^{\wedge}, \mathrm{J}^{\wedge}\right)$ is obtained from $(\nu, J)$ by adding a singular string of length $\mu_{L}$ to each of the first $\eta_{L}-1$ rigged partitions in $(\nu, J)$. Let $m^{\wedge}$ be the matrix associated to $\nu^{\wedge}$. Then

$$
m_{i j}^{\wedge}=m_{i j}+\chi\left(1 \leq j \leq \mu_{L}\right)\left\{\chi\left(i=\eta_{L}\right)-\chi(i=1)\right\} .
$$

Using furthermore that

$$
\begin{aligned}
\sum_{a=1}^{L+1} \chi\left((i, j) \in R_{a}^{\wedge}\right) & =\sum_{a=1}^{L} \chi\left((i, j) \in R_{a}\right) \\
& +\chi\left(1 \leq i \leq \mu_{L}\right)\left\{\chi(j=1)-\chi\left(j=\eta_{L}\right)\right\}
\end{aligned}
$$

one finds by (7.2) that $m_{i j}^{\wedge t}=m_{i j}^{t}$. Since $\jmath^{>}$is an inclusion it follows that the configurations of $\operatorname{tr}_{\mathrm{RC}} \circ \jmath^{\wedge}(\nu, J)$ and $\jmath^{>} \circ \operatorname{tr}_{\mathrm{RC}}(\nu, J)$ coincide. Since $\jmath^{\wedge}$ preserves the vacancy numbers by Lemma 3.9 also the riggings coincide.

Lemma 7.3. Suppose the last rectangle of $R$ consists of a single column. Then the following diagram commutes:

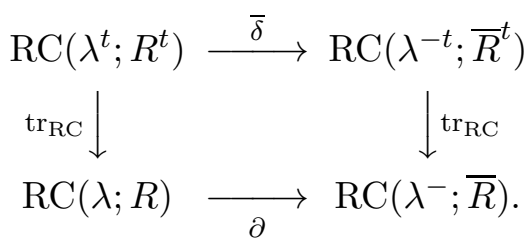

The proof of this Lemma is given in Appendix B.

Proof of Theorem 7.1. If $R$ is empty the result holds trivially. If $R_{L}$ has more than one column consider the diagram:

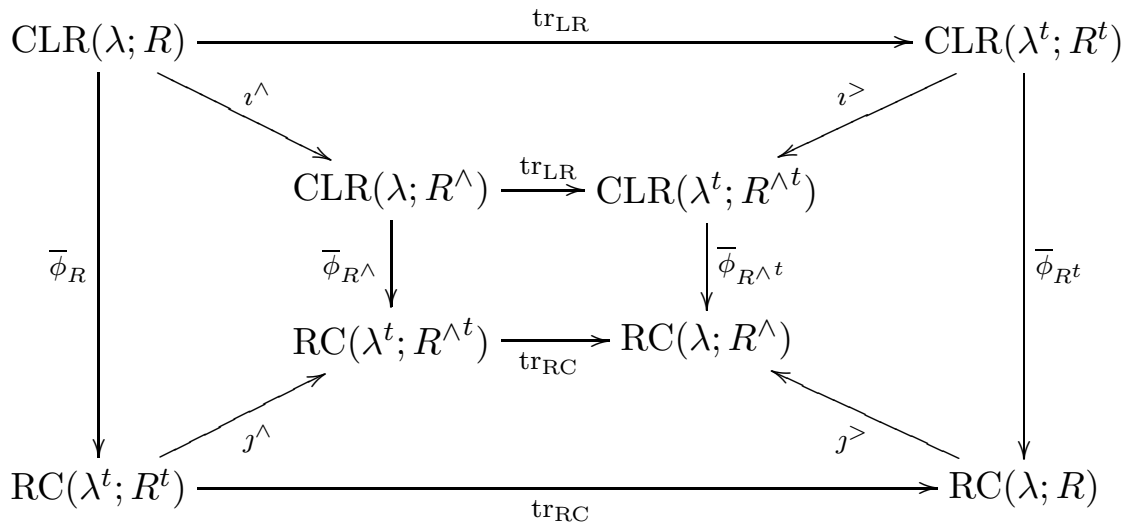

Since $\operatorname{tr}_{\mathrm{LR}}$ is an involution the top face commutes by (6.1). The bottom face commutes by Lemma 7.2 and the back face by induction. The left face is the commutative diagram (4.3) and the right face that of Lemma 6.2. Since $\jmath^{>}$is injective the front face commutes by Lemma 5.3 . 
Suppose the last rectangle is a single column. Consider the diagram:

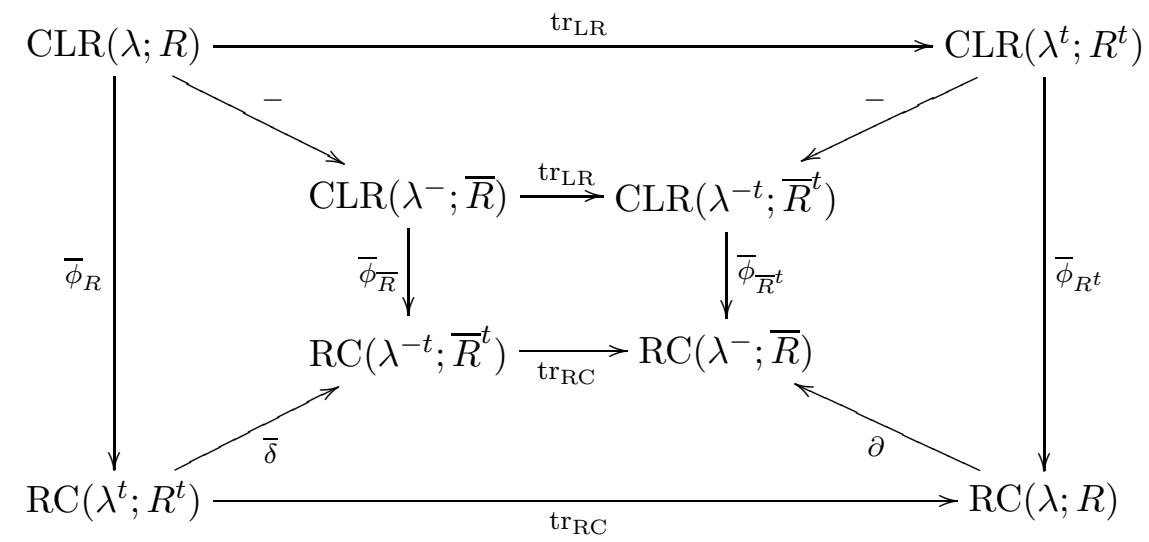

$\partial$ is injective, the back face commutes by induction, the commutativity of the top is trivial and that of the bottom face follows from Lemma 7.3. The left face commutes by (4.2) and the right face by (6.6) with $\lambda$ and $R$ replaced by their transposes. So by Lemma 5.3 the front face commutes.

\section{EMBEDDINGS}

In [20, Section 6.1] and [23, Section 2.3] embeddings were given between sets of LR tableaux of the form $\operatorname{LRT}(\lambda ; R)$. We restate these embeddings in terms of the tableaux $\operatorname{CLR}(\lambda ; R)$ and show in Theorem 8.3 that they are induced by inclusions of the corresponding sets of rigged configurations under the coquantum version $\widetilde{\phi}$ of the map $\bar{\phi}$ (see (4.1)), thereby proving [13, Conjecture 18].

8.1. Embedding definitions. Define the partial order $\lambda \unrhd \mu$ on partitions by $|\lambda|=|\mu|$ and $\lambda_{1}+\cdots+\lambda_{i} \geq \mu_{1}+\cdots+\mu_{i}$ for all $i$. Let $R$ and $R^{\prime}$ be two sequences of rectangles. Recall that $\xi^{(k)}(R)$ is the partition whose parts are the heights of the rectangles in $R$ of width $k$. Say that $R \unrhd R^{\prime}$ if $\xi^{(k)}(R) \unrhd \xi^{(k)}\left(R^{\prime}\right)$ for all $k \geq 1$. Clearly $R \unrhd R^{\prime}$ and $R^{\prime} \unrhd R$ if and only if $R^{\prime}$ is a reordering of $R$. Thus the relation $R \unrhd R^{\prime}$ is a pseudo-order. It is generated by the following two relations (see [23, Section 2.3])

(E1) $R \unrhd R^{+}$where $R_{i}=R_{i}^{+}$for $i>2, R_{1}=\left(c^{a}\right), R_{2}=\left(c^{b}\right), R_{1}^{+}=\left(c^{a-1}\right)$, $R_{2}^{+}=\left(c^{b+1}\right)$ for $a-1 \geq b+1$ and $c$ a positive integer.

(E2) $R \unrhd s_{p} R$ where $s_{p} R$ denotes the sequence obtained from $R$ by exchanging the rectangles $R_{p}$ and $R_{p+1}$. 
For the relation (E1) define the embedding $\imath^{+}$by the commutation of the diagram

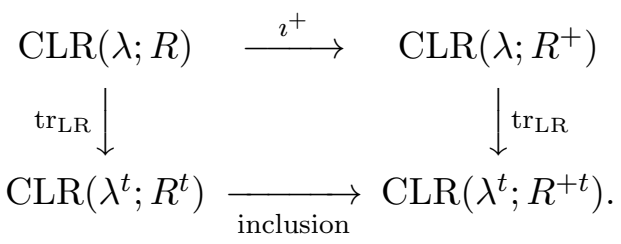

For the relation (E2) we have the following result.

Definition-Proposition 8.1. For $1 \leq p \leq L-1$ there are unique bijections $\sigma_{p}: \operatorname{CLR}(\lambda ; R) \rightarrow \operatorname{CLR}\left(\lambda ; s_{p} R\right)$ satisfying the following properties:

1. If $p<L-1$ then $\sigma_{p}$ commutes with restriction to the initial interval $B-B_{L}$ where $B$ and $B_{L}$ are as in the definition of $\operatorname{CLR}(\lambda ; R)$.

2. If $p=L-1$ then the following diagram commutes:

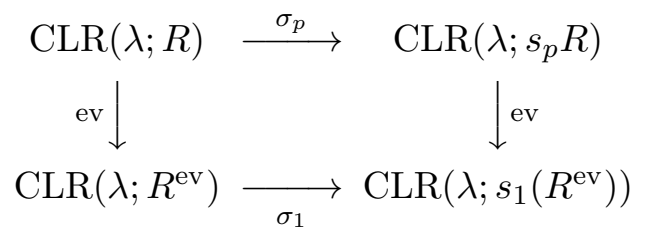

Proof. One may reduce to the case $p=L-1$ using 1 , then to the case $p=1$ using 2 , and then to the case $p=1$ and $L=2$ using 1 again. In this case the sets of tableaux are all empty or all singletons by [22, Prop. 33] and the result holds trivially.

Remark 8.2. In the case that $R_{j}$ is a single row of length $\eta_{j}$ for all $j$, by Example 2.9 there is a bijection of $\operatorname{CLR}(\lambda ; R)$ with the set of columnstrict tableaux of shape $\lambda$ and content $\eta$. The action of the bijections $\sigma_{p}$ on the column-strict tableaux are the automorphisms of conjugation defined by Lascoux and Schützenberger 17].

Let $R \unrhd R^{\prime}$. Then $R^{\prime}$ may be obtained from $R$ by a sequence of transformations of the form (E1) and (E2); fix such a sequence. Define the embedding $i_{R}^{R^{\prime}}: \operatorname{CLR}(\lambda ; R) \rightarrow \operatorname{CLR}\left(\lambda ; R^{\prime}\right)$ as the corresponding composition of embeddings of the form $\imath^{+}$and $\sigma_{p}$. By [23, Theorem 4] it follows that the embedding $i_{R}^{R^{\prime}}$ is independent of the sequence of transformations (E1) and (E2) leading from $R$ to $R^{\prime}$. This fact also follows immediately from Theorem 8.3 below.

For rigged configurations, it follows immediately from the definitions that if $R \unrhd R^{\prime}$ then there is an inclusion $\mathrm{RC}\left(\lambda^{t} ; R^{t}\right) \subseteq \mathrm{RC}\left(\lambda^{t} ;\left(R^{\prime}\right)^{t}\right)$ which shall be denoted by $j_{R}^{R^{\prime}}$. 
Theorem 8.3 (Embedding Theorem). Let $R \unrhd R^{\prime}$. Then the diagram commutes:

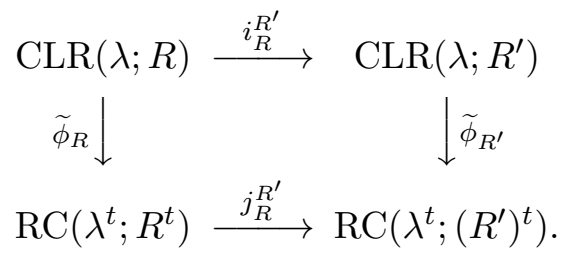

Clearly it suffices to prove Theorem 8.3 in the cases (E1) and (E2).

8.2. The case (E1). Suppose $R \unrhd R^{+}$as in (E1). Define the embedding $\jmath^{+}: \mathrm{RC}\left(\lambda^{t} ; R^{t}\right) \rightarrow \mathrm{RC}\left(\lambda^{t} ; R^{+t}\right)$ by the commutativity of the diagram

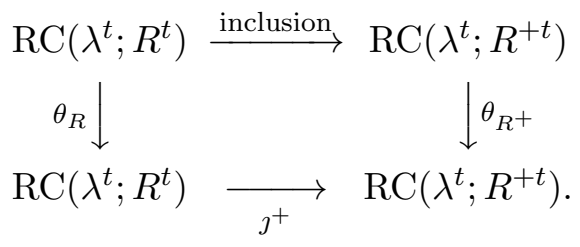

Note that $j^{+}$preserves colabels. The following result immediately proves Theorem 8.3 in the case (E1).

Lemma 8.4. The diagram commutes:

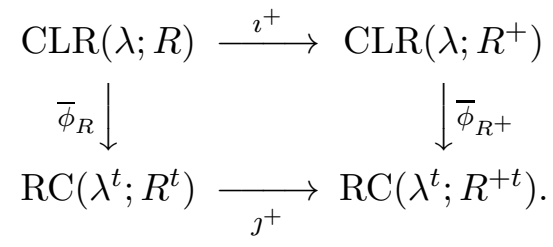

Proof. For $L \geq 3$ the proof of this lemma is the same as the proof of Lemma 6.1 with $R^{<}$(resp. $\left.\imath^{<}, \jmath^{<}\right)$replaced by $R^{+}\left(\operatorname{resp} . \imath^{+}, \jmath^{+}\right)$. Note that in the remaining case $L \leq 2$ both $R$ and $R^{+}$have at most two rectangles so that $\operatorname{CLR}(\lambda ; R)$ and $\operatorname{CLR}\left(\lambda ; R^{+}\right)$are singletons, and the proof follows again by arguments similar to those of the proof of Lemma 6.1.

8.3. The case (E2). The case (E2) of Theorem 8.3 is an immediate consequence of the following result.

Lemma 8.5. The diagram commutes:

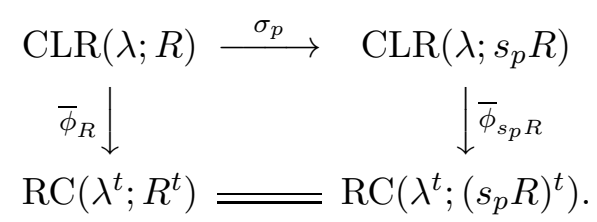

Proof. Recall the intervals of integers $B_{j}(1 \leq j \leq L)$ in the definition of the set $\operatorname{CLR}(\lambda ; R)$. It follows from the definition of $\sigma_{p}$ that for all $S \in$ $\operatorname{CLR}(\lambda ; R),\left.\left(\sigma_{p} S\right)\right|_{B_{j}}=\left.S\right|_{B_{j}}$ for $j>p+1$. Using this fact and the definition 
of $\bar{\phi}_{R}$, one may reduce to the case $L=p+1$. So it may be assumed that $L=p+1$. Obviously $\left(s_{p} R\right)^{\mathrm{ev}}=s_{1}\left(R^{\mathrm{ev}}\right)$. Consider the diagram

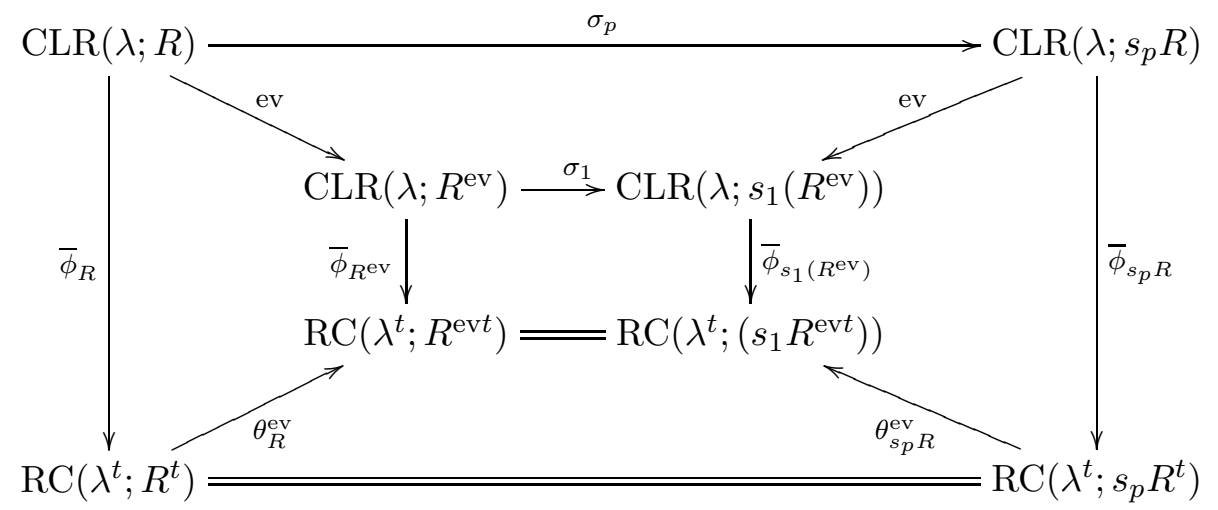

The commutation of the bottom face is obvious. The top face commutes by definition and the left and right faces commute by Theorem 5.6. The desired commutation of the front face is reduced to the commutation of the back face. Replacing $R$ by $R^{\text {ev }}$ it may now be assumed that $p=1$. Applying a previous reduction it may also be assumed that $L=2$. But in this case the sets in (8.4) are all singletons or all empty, so the diagram (8.4) commutes.

8.4. Connection with $\operatorname{LRT}(\lambda ; R)$. In [13, Section 10], [20, Section 6.1] and [23, Section 2.3] embeddings $\theta_{R}^{R^{\prime}}: \operatorname{LRT}(\lambda ; R) \rightarrow \operatorname{LRT}\left(\lambda ; R^{\prime}\right)$ were defined when $R \unrhd R^{\prime}$. They are related to the embeddings $i_{R}^{R^{\prime}}: \operatorname{CLR}(\lambda ; R) \rightarrow$ $\operatorname{CLR}\left(\lambda ; R^{\prime}\right)$ by the bijection $\beta_{R}: \operatorname{LRT}(\lambda ; R) \rightarrow \operatorname{CLR}(\lambda ; R)$ of Remark 2.6.

Proposition 8.6. Let $R \unrhd R^{\prime}$. The diagram commutes:

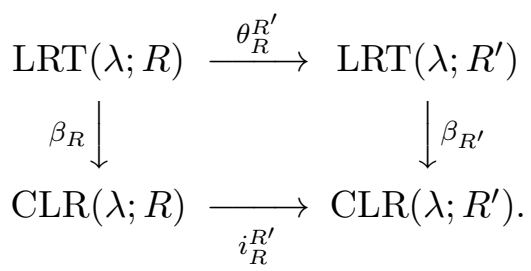

For the proof recall the evacuation map $\operatorname{Ev}: \operatorname{LRT}(\lambda ; R) \rightarrow \operatorname{LRT}\left(\lambda ; R^{\mathrm{ev}}\right)$. Let $n=\sum_{j=1}^{L} \mu_{j}$ be the sum of heights of rectangles in $R$. There is a unique involution $T \mapsto T^{\mathrm{Ev}}$ on column-strict tableaux of shape $\lambda$ in the alphabet $[1, n]$ defined by

$$
\operatorname{shape}\left(\left.\left(T^{\mathrm{Ev}}\right)\right|_{[1, i]}\right)=\operatorname{shape}\left(P\left(\left.T\right|_{[n-i+1, n]}\right)\right)
$$

for all $1 \leq i \leq n$. The bijection Ev restricts to a bijection $\operatorname{LRT}(\lambda ; R) \rightarrow$ $\operatorname{LRT}\left(\lambda ; R^{\mathrm{ev}}\right) 13$. 
Lemma 8.7. The diagram commutes:

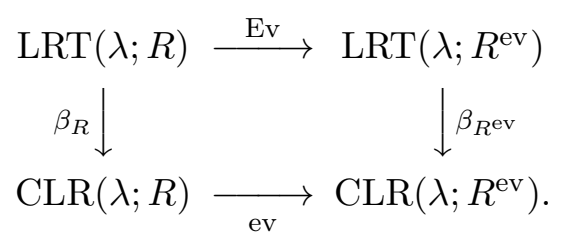

Sketch of Proof. $\beta_{R}=\gamma_{R}^{-1}$ o std by Remark 2.6. Using the well-known fact std $\circ \mathrm{Ev}=$ evostd it is enough to show that the following diagram commutes:

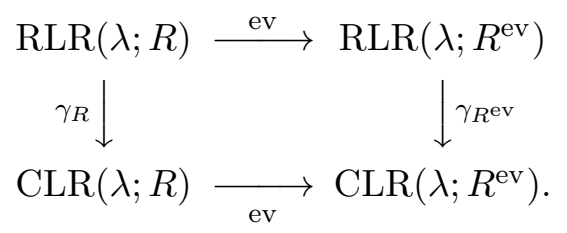

By 17] $S^{\mathrm{ev}}=P(\#$ word $(S))$ for $S \in \mathrm{ST}(\lambda)$ where \#w is the reverse of the complement of the word $w$ in the alphabet $[1,|\lambda|]$. Let $\operatorname{WR}(R)$ (resp. $\mathrm{WC}(R))$ be the set of standard words $w$ such that the standard tableau $P(w)$ is in $\operatorname{RLR}(\lambda ; R)$ (resp. $\operatorname{CLR}(\lambda ; R)$ ). The relabeling bijection $\gamma_{R}$ : $\operatorname{RLR}(\lambda ; R) \rightarrow \operatorname{CLR}(\lambda ; R)$ extends to a map $\mathrm{WR}(R) \rightarrow \mathrm{WC}(R)$ in an obvious way. This relabeling map satisfies $P\left(\gamma_{R}(w)\right)=\gamma_{R}(P(w))$ [23, Lemma 36].

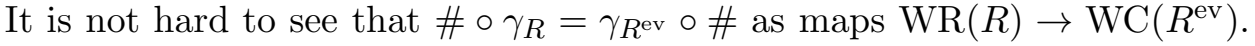
For all $S \in \operatorname{RLR}(\lambda ; R)$ we have

$$
\begin{aligned}
\left(\gamma_{R}(S)\right)^{\mathrm{ev}} & =P\left(\# \gamma_{R}(S)\right)=P\left(\# \gamma_{R}(\operatorname{word}(S))\right) \\
& =P\left(\gamma_{R^{\mathrm{ev}}}(\# \operatorname{word}(S))\right)=\gamma_{R^{\mathrm{ev}}}(P(\# \operatorname{word}(S))) \\
& =\gamma_{R^{\mathrm{ev}}}\left(S^{\mathrm{ev}}\right) .
\end{aligned}
$$

Proof of Proposition 8.9. Again the result follows from the special cases (E1) and (E2). Consider the case (E1). By the definition of the relabeling map $\beta_{R}$ and the fact that in this case both $\theta_{R}^{R^{\prime}}$ and $i_{R}^{R^{\prime}}$ only change the subtableaux corresponding to the first two rectangles, one may reduce to the case that $L=2$. By [22, Prop. 33] all sets are either singletons or empty. The vertical maps are bijections and the horizontal maps are embeddings, so the diagram must commute.

Denote by $s_{p}$ the rectangle switching bijection on $\operatorname{LRT}(\lambda ; R)$ as defined in [13, Section 7] (see also [22, Section 2.5]). In the case (E2), neither $s_{p}$ nor $\sigma_{p}$ disturb the subtableaux corresponding to the rectangles $R_{j}$ for $j>p+1$. Therefore it may be assumed that $L=p+1$. Now the evacuation map is applied to the diagram (8.5): 


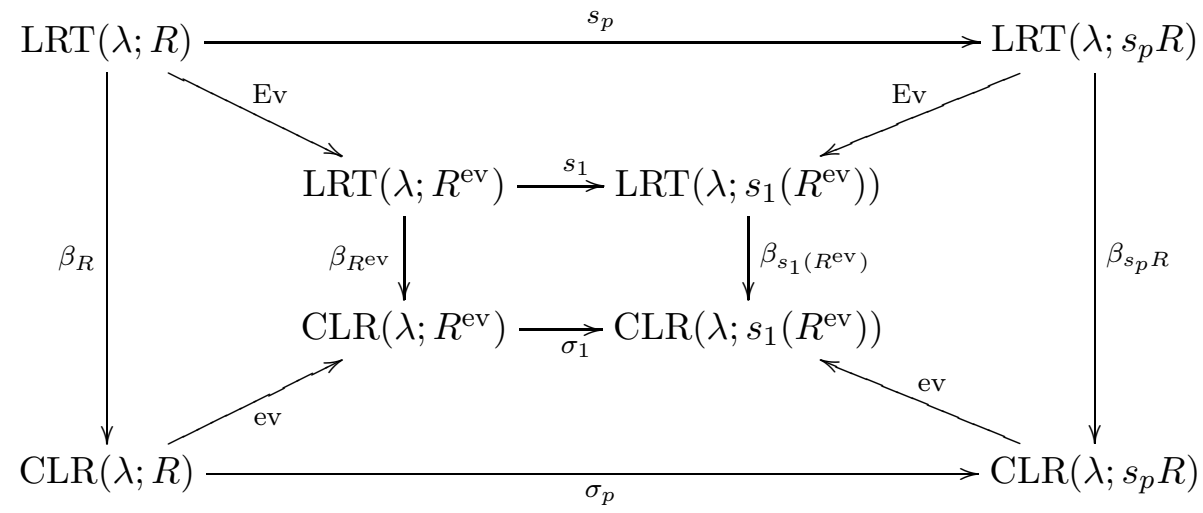

The left and right faces commute by Lemma 8.7. The top face commutes by the proof of [13, Lemma 5], and the bottom commutes by the definition of $\sigma_{p}$. To obtain the desired commutation of the front face, it is enough to show that the back face commutes. But the back face is the special case $p=1$ with $R$ replaced by $R^{\mathrm{ev}}$. This reduces to the case $p=1$. As before one may reduce to the case that $L=p+1=2$. But in this case by [22, Prop. 33 the sets are all singletons or all empty, so the diagram commutes.

8.5. Single rows. Let $\operatorname{rows}(R)$ be obtained by slicing all of the rectangles of $R$ into single rows. Clearly $R \unrhd \operatorname{rows}(R)$ and $\operatorname{rows}(R)$ is minimal with respect to the pseudo-order $\unrhd$. Define $i_{R}:=i_{R}^{\text {rows }(R)}: \operatorname{CLR}(\lambda ; R) \rightarrow$ $\operatorname{CLR}(\lambda ; \operatorname{rows}(R))$. Recall that Schensted's standardization map gives a bijection from column-strict tableaux of shape $\lambda$ and content $\left(\eta_{1}^{\mu_{1}}, \eta_{2}^{\mu_{2}}, \ldots, \eta_{L}^{\mu_{L}}\right)$ onto $\operatorname{CLR}(\lambda ; \operatorname{rows}(R))$, so that $i_{R}$ essentially embeds $\operatorname{CLR}(\lambda ; R)$ into columnstrict tableaux.

\section{Statistics For LR tableaux And Rigged CONFigurations}

Recall that both the set of LR tableaux and the set of rigged configurations are endowed with statistics, given by the charge $c_{R}(T)$ for $T \in$ $\operatorname{CLR}(\lambda ; R)$ and $c c(\nu, J)$ for $(\nu, J) \in \operatorname{RC}\left(\lambda^{t} ; R^{t}\right)$. The statistic $c c(\nu, J)$ was given in (2.8) and an explicit expression for the generalized charge $c_{R}(T)$ can be found in [22, (2.1)].

The objective of this section is to prove that $\widetilde{\phi}$ preserves the statistics, which settles [13, Conjecture 9].

Theorem 9.1. Let $T \in \operatorname{CLR}(\lambda ; R)$. Then $c_{R}(T)=c c\left(\widetilde{\phi}_{R}(T)\right)$.

Proof. Recall the embedding $i_{R}: \operatorname{CLR}(\lambda ; R) \rightarrow \operatorname{CLR}(\lambda ; \operatorname{rows}(R))$ of Section 8.5. By 23, Prop. 7], for all $S \in \operatorname{CLR}(\lambda ; R)$ we have $c_{\operatorname{rows}(R)}\left(i_{R}(S)\right)=$ $c_{R}(S)$. On the other hand by Theorem 8.3, $\widetilde{\phi}_{\text {rows }(R)}\left(i_{R}(S)\right)=\widetilde{\phi}_{R}(S)$. By replacing $R$ by $\operatorname{rows}(R)$ it may be assumed that $R$ consists of single rows. 
The transpose maps are applied to reduce to the case of single columns. Recall the definition of the number $n(R)$ [13, (2.7)]. For all $S \in \operatorname{CLR}(\lambda ; R)$ we have $c_{R^{t}}\left(\operatorname{tr}_{\mathrm{LR}}(S)\right)=n(R)-c_{R}(S)$ by [23, Prop. 24 and Theorem 26] and [20, Lemma 6.5]. For all $(\nu, J) \in \mathrm{RC}\left(\lambda^{t} ; R^{t}\right)$ we have $c c\left(\operatorname{tr}_{\mathrm{RC}}(\nu, J)\right)=$ $n(R)-c c(\nu, J)$ by [13, $(9.12)]$. Moreover

$$
\begin{aligned}
\operatorname{tr}_{\mathrm{RC}} \circ \widetilde{\phi}_{R} & =\operatorname{tr}_{\mathrm{RC}} \circ \theta_{R} \circ \bar{\phi}_{R}=\theta_{R^{t}} \circ \operatorname{tr}_{\mathrm{RC}} \circ \bar{\phi}_{R} \\
& =\theta_{R^{t}} \circ \bar{\phi}_{R^{t}} \circ \operatorname{tr}_{\mathrm{LR}}=\widetilde{\phi}_{R^{t}} \circ \operatorname{tr}_{\mathrm{LR}}
\end{aligned}
$$

by the definitions of $\widetilde{\phi}_{R}, \operatorname{tr}_{R C}, \theta_{R}$, and Theorem 7.1. From these facts, by replacing $R$ with $R^{t}$ it may be assumed that $R$ consists of single columns.

Applying the first reduction again, it may be assumed that $R$ consists of single cells. Observe that in this case $\operatorname{CLR}(\lambda ; R)=\mathrm{ST}(\lambda)$. It is possible to verify $c_{R}(S)=\operatorname{cc}\left(\widetilde{\phi}_{R}(S)\right)$ for $S \in \mathrm{ST}(\lambda)$ directly. It is known that for all $S \in \mathrm{ST}(\lambda), c_{R}(S)=c(S)$ where $c(S)$ is the ordinary charge of a standard tableau $S$ [18, Section III.6]. Let asc $(S)$ denote the number of ascents in $S$, that is, the number of indices $i$ such that $i+1$ is in a later column in $S$ than $i$. It follows immediately from the definition of $c$ that $c\left(S^{-}\right)=c(S)-\operatorname{asc}(S)$. By induction it suffices to show that if $\widetilde{\phi}_{R}(S)=(\nu, J)$ then $\operatorname{asc}(S)$ is equal to $\alpha_{1}^{(1)}$ and that $c c(\nu, J)-c c(\widetilde{\delta}(\nu, J))=\alpha_{1}^{(1)}$.

It is first shown that $\operatorname{asc}(S)=\alpha_{1}^{(1)}$. Denote $(\widetilde{\nu}, \widetilde{J})=\widetilde{\delta}(\nu, J)$, let $\widetilde{\alpha}_{n}^{(k)}$ be the size of the $n$-th column in $\widetilde{\nu}^{(k)}$ and let $\widetilde{\ell}^{(k)}$ be the length of the selected string of label zero in $(\nu, J)^{(k)}$ in passing to $(\widetilde{\nu}, \widetilde{J})^{(k)}$. By induction $\operatorname{asc}\left(S^{-}\right)=\widetilde{\alpha}_{1}^{(1)}$ where $\widetilde{\phi}_{\widetilde{R}}\left(S^{-}\right)=\widetilde{\delta}(\nu, J)=(\widetilde{\nu}, \widetilde{J})$. (At this point the astute reader may be concerned that the relation between $\widetilde{\phi}_{R}$ and $\bar{\phi}_{R}$ involves $\theta_{R}$ whereas the relation between $\bar{\delta}$ and $\widetilde{\delta}$ involves $\theta_{R}^{\text {ev }}$ (compare (3.15)); however, since $R$ consists only of single boxes $\left.\theta_{R}=\theta_{R}^{\mathrm{ev}}\right)$. Now

$$
\alpha_{1}^{(1)}-\widetilde{\alpha}_{1}^{(1)}=\chi\left(\widetilde{\ell}^{(1)}=1\right)
$$

Thus it must be shown that $\widetilde{\ell}^{(1)}=1$ if and only if $N-1$ is an ascent of $S$ where $N=|\lambda|$. Let $c$ and $c^{-}$be the column indices of the letters $N$ and $N-1$ in $S$ and $\widetilde{\widetilde{\ell}}^{(k)}$ the length of the string selected by $\widetilde{\delta}$ in $(\widetilde{\nu}, \widetilde{J})^{(k)}$. First suppose that $\widetilde{\ell}^{(1)}=1$. Then $\widetilde{\widetilde{\ell}}^{(k)} \geq \widetilde{\ell}^{(k)}$ for all $0 \leq k<c$. Hence $c>c^{-}$by (3.16) with $k=c-1$. Conversely, if $\widetilde{\ell}^{(1)}>1$ then $\widetilde{\widetilde{\ell}}^{(k)}<\widetilde{\ell}^{(k)}$ for all $0 \leq k<c$ so that $c \leq c^{-}$. This proves $\operatorname{asc}(S)=\alpha_{1}^{(1)}$. 
Now it is shown that $c c(\nu, J)-c c(\widetilde{\delta}(\nu, J))=\alpha_{1}^{(1)}$ when $R$ consists of single cells. To this end we first compute

$$
\begin{aligned}
c c(\nu)-c c(\widetilde{\nu}) & =\sum_{k, n \geq 1} \alpha_{n}^{(k)}\left(\alpha_{n}^{(k)}-\alpha_{n}^{(k+1)}\right) \\
& -\sum_{k, n \geq 1}\left(\alpha_{n}^{(k)}-\delta_{n, \widetilde{\ell}^{(k)}}\right)\left(\alpha_{n}^{(k)}-\alpha_{n}^{(k+1)}-\delta_{n, \widetilde{\ell}^{(k)}}+\delta_{\left.n, \widetilde{\ell}^{(k+1)}\right)}\right) \\
& =\sum_{k \geq 1}\left(2 \alpha_{\widetilde{\ell}^{(k)}}^{(k)}-\alpha_{\widetilde{\ell}^{(k+1)}}^{(k)}-\alpha_{\widetilde{\ell}^{(k)}}^{(k+1)}-\chi\left(\widetilde{\ell}^{(k)}<\widetilde{\ell}^{(k+1)}\right)\right) .
\end{aligned}
$$

Next let us determine the difference of the sums of quantum numbers. Recall that $\widetilde{\delta}$ does not change the colabels of unselected strings, and that the vacancy numbers change by (3.16). The selected strings have label zero before and after being shortened. Then

$$
\begin{aligned}
& \sum_{k, n \geq 1}\left|J_{n}^{(k)}\right|-\sum_{k, n \geq 1}\left|\widetilde{\mathrm{J}}_{n}^{(k)}\right| \\
& =\sum_{k, n \geq 1}\left(m_{n}\left(\nu^{(k)}\right)-\delta_{n, \widetilde{\ell}^{(k)}}\right)\left(\chi\left(\widetilde{\ell}^{(k-1)} \leq n<\widetilde{\ell}^{(k)}\right)-\chi\left(\widetilde{\ell}^{(k)} \leq n<\widetilde{\ell}^{(k+1)}\right)\right) \\
& =\sum_{k \geq 1}\left(\alpha_{\widetilde{\ell}^{(k-1)}}^{(k)}-\alpha_{\widetilde{\ell}^{(k)}}^{(k)}\right)-\sum_{k \geq 1}\left(\alpha_{\widetilde{\ell}^{(k)}}^{(k)}-\alpha_{\widetilde{\ell}^{(k+1)}}^{(k)}\right)+\sum_{k \geq 1} \chi\left(\widetilde{\ell}^{(k)}<\widetilde{\ell}^{(k+1)}\right) .
\end{aligned}
$$

By (9.1) and (9.2) we have $c c(\nu, J)-c c(\widetilde{\nu}, \widetilde{J})=\alpha_{\widetilde{\ell}^{(0)}}^{(1)}=\alpha_{1}^{(1)}$.

The cocharge of an LR tableau $T \in \operatorname{CLR}(\lambda ; R)$ is defined as $\operatorname{co}_{R}(T)=$ $\|R\|-c_{R}(T)$ where $\|R\|=\sum_{i<j}\left|R_{i} \cap R_{j}\right|$. Recall that for $\lambda$ and $\mu$ partitions $\operatorname{CST}(\lambda ; \mu)$ denotes the set of column-strict tableaux of shape $\lambda$ and content $\mu$. By Example 2.9 the set $\operatorname{CST}(\lambda ; \mu)$ equals $\operatorname{LRT}(\lambda ; R)$ where $R=\left(\left(\mu_{1}\right), \ldots,\left(\mu_{L}\right)\right)$, and identifying the two sets the cocharge of a columnstrict tableau $T$ is given by $\operatorname{co}(T)=\operatorname{co}_{R}(T)$.

Recall the map $\beta_{R}: \operatorname{LRT}(\lambda ; R) \rightarrow \operatorname{CLR}(\lambda ; R)$ of Remark 2.6. Then, identifying $\operatorname{CST}(\lambda ; \mu)$ and $\operatorname{LRT}(\lambda ; R)$, the coquantum version of the original bijection 12 between column-strict tableaux and rigged configurations is given by $\phi_{R^{t}} \circ \operatorname{tr}_{\mathrm{LR}} \circ \beta_{R}$.

Corollary 9.2. Let $T$ be a column-strict tableau of shape $\lambda$ and partition content $\mu=\left(\mu_{1}, \ldots, \mu_{L}\right)$ and $1 \leq r \leq L$ an index such that $\mu_{r}>\mu_{r+1}$ where $\mu_{L+1}=0$. Let $T^{\prime}$ be obtained from $T$ by applying the automorphism of conjugation (see Remark 8.8) $\sigma=\sigma_{L-1} \sigma_{L-2} \ldots \sigma_{r}$ that changes the content to $\left(\mu_{1}, \ldots, \mu_{r-1}, \mu_{r+1}, \ldots, \mu_{L}, \mu_{r}\right)$, then removing the rightmost copy of the letter $L$, then applying the automorphism of conjugation $\sigma^{-1}$ to change the content to $\left(\mu_{1}, \ldots, \mu_{r-1}, \mu_{r}-1, \mu_{r+1}, \ldots, \mu_{L}\right)$. Let $R=\left(\left(\mu_{1}\right), \ldots,\left(\mu_{L}\right)\right)$ and $(\nu, J)=\widetilde{\phi}_{R^{t}} \circ \operatorname{tr}_{\mathrm{LR}} \circ \beta_{R}(T)$. Then

$$
\operatorname{co}(T)-\operatorname{co}\left(T^{\prime}\right)=\alpha_{\mu_{r}}^{(1)}
$$


where $\alpha_{\mu_{r}}^{(1)}$ is the size of the $\mu_{r}$-th column of $\nu^{(1)}$.

Proof. By Lemma 8.5 it suffices to prove the result in the case that $\mu=$ $\left(\mu_{1}, \ldots, \mu_{L}\right)$ is any sequence of positive integers, $r=L$, and $T^{\prime}$ is obtained from $T$ by removing the rightmost copy of the letter $L$. Set $S=\operatorname{tr}_{\mathrm{LR}} \circ \beta_{R}(T)$, $S^{\prime}=\operatorname{tr}_{\mathrm{LR}} \circ \beta_{\bar{R}^{t}}\left(T^{\prime}\right),(\nu, J)=\widetilde{\phi}_{R^{t}}(S)$ and $\left(\nu^{\prime}, J^{\prime}\right)=\widetilde{\phi}_{\overline{R^{t}}}\left(S^{\prime}\right)$. By [20, Lemma 6.5] and [23, Theorem 26] $\operatorname{co}_{R}\left(\operatorname{tr}_{\mathrm{LR}}(S)\right)=c_{R^{t}}(S)$. Hence

$$
\begin{aligned}
\operatorname{co}(T)-\operatorname{co}\left(T^{\prime}\right) & =c_{R^{t}}(S)-c \overline{R^{t}}\left(S^{\prime}\right) \\
& =c c\left(\widetilde{\phi}_{R^{t}}(S)\right)-c c\left(\widetilde{\phi}_{\overline{R^{t}}}\left(S^{\prime}\right) \quad \text { by Theorem } 9.1\right. \\
& =c c(\nu, J)-c c\left(\nu^{\prime}, J^{\prime}\right) .
\end{aligned}
$$

Notice that $(\nu, J) \in \operatorname{RC}(\lambda ; R)$ where $R^{t}$ is a sequence of single columns. Similar calculations to (9.1) and (9.2) yield $c c(\nu, J)-c c\left(\nu^{\prime}, J^{\prime}\right)=\alpha_{\mu_{L}}^{(1)}$.

\section{Appendix A. Proof of Lemma 3.13}

The proof of Lemma 3.13 is given here by Lemmas A.2 and A.3. After the Lemmas it is explained why the statements regarding $\overline{\mathrm{rk}}$ and rk in Lemma 3.13 follow from Lemma A.2.

In this section the following notation is used. Let $R=\left(R_{1}, \ldots, R_{L}\right)$ be a sequence of rectangles with $R_{i}=\left(\eta_{i}^{\mu_{i}}\right)$, such that $R_{1}$ and $R_{L}$ are single columns and $|R| \geq 2$, let $\lambda$ be a partition and $(\nu, J) \in \operatorname{RC}\left(\lambda^{t} ; R^{t}\right)$. Write

$$
\begin{aligned}
\bar{\delta}(\nu, J) & =(\bar{\nu}, \overline{\mathrm{J}}) \\
\widetilde{\delta}(\nu, J) & =(\widetilde{\nu}, \widetilde{\mathrm{J}}) \\
\widetilde{\delta} \circ \bar{\delta}(\nu, J) & =(\widetilde{\bar{\nu}}, \widetilde{\bar{J}}) \\
\bar{\delta} \circ \widetilde{\delta}(\nu, J) & =(\overline{\widetilde{\nu}}, \overline{\widetilde{J}}) .
\end{aligned}
$$

Furthermore, let $\bar{\ell}^{(k)}, \widetilde{\ell}^{(k)}, \widetilde{\bar{\ell}}^{(k)}$ and $\overline{\tilde{\ell}}^{(k)}$ denote the lengths of the strings that are shortened in the transformations $(\nu, J) \mapsto(\bar{\nu}, \bar{J}),(\nu, J) \mapsto(\widetilde{\nu}, \widetilde{J})$, $(\bar{\nu}, \overline{\mathrm{J}}) \mapsto(\widetilde{\bar{\nu}}, \widetilde{\bar{J}})$ and $(\widetilde{\nu}, \widetilde{\mathrm{J}}) \mapsto(\overline{\widetilde{\nu}}, \overline{\widetilde{J}})$, respectively.

Remark A.1. Note that, except for the strings that change length in the transformations, a string has label zero before applying $\bar{\delta}$ if and only it does afterwards, and a string is singular before applying $\widetilde{\delta}$ if and only if it is afterwards.

If $\bar{\delta}$ and $\widetilde{\delta}$ select the same string in $\nu^{(k)}$ (that is, both select a string with the same length and same label), say that the doubly singular case holds for $\nu^{(k)}$. Otherwise say that the generic case holds for $\nu^{(k)}$.

The next Lemma shows that $\overline{\widetilde{\nu}}=\widetilde{\bar{\nu}}$.

Lemma A.2. If the generic case holds for $\nu^{(k)}$ then $\bar{\ell}^{(k)}=\overline{\bar{\ell}}^{(k)}$ and $\widetilde{\ell}^{(k)}=$ $\widetilde{\bar{\ell}}^{(k)}$. Otherwise suppose the doubly singular case holds for $\nu^{(k)}$. Let $\ell:=$ $\bar{\ell}^{(k)}=\widetilde{\ell}^{(k)}$ be the common string length. Then 
1. If $\widetilde{\bar{\ell}}^{(k)}<\ell$ (or $\overline{\bar{\ell}}^{(k)}<\ell$ ) then $\widetilde{\bar{\ell}}^{(k)}=\overline{\tilde{\ell}}^{(k)}=\ell-1$, and $m_{\ell-1}\left(\nu^{(k+1)}\right)=0$.

2. If $\widetilde{\bar{\ell}}^{(k)}=\ell$ (or $\overline{\widetilde{\ell}}^{(k)}=\ell$ ) then $\overline{\bar{\ell}}^{(k)}=\widetilde{\bar{\ell}}^{(k)}=\ell$.

3. If $\widetilde{\bar{\ell}}^{(k)}>\ell$ (or $\overline{\bar{\ell}}^{(k)}>\ell$ ) then $\widetilde{\bar{\ell}}^{(k)}=\overline{\bar{\ell}}^{(k)}, \widetilde{\ell}^{(k+1)} \geq \widetilde{\bar{\ell}}^{(k)}$ and $\bar{\ell}^{(k+1)} \geq \widetilde{\bar{\ell}}^{(k)}$. Moreover, if $\widetilde{\bar{\ell}}^{(k)}=\infty$ then $\lambda_{k}^{t}=\lambda_{k+1}^{t}$.

Lemma A.3. $\widetilde{\bar{J}}=\overline{\widetilde{J}}$.

Together Lemmas A.2 and A.3 establish the desired result that $(\overline{\widetilde{\nu}}, \overline{\widetilde{J}})=$ $(\widetilde{\bar{\nu}}, \widetilde{\bar{J}})$.

Given Lemma A.2, the assertions of Lemma 3.13 on the relationships between $\bar{r}=\overline{\mathrm{rk}}(\nu, J), \widetilde{r}=\widetilde{\mathrm{rk}}(\nu, J), \widetilde{\bar{r}}=\widetilde{\mathrm{rk}}(\bar{\delta}(\nu, J))$, and $\overline{\widetilde{r}}=\overline{\mathrm{rk}}(\widetilde{\delta}(\nu, J))$ are now established.

Suppose $\bar{r}<\widetilde{r}$. Then $\bar{\ell}^{(\bar{r})}=\infty>\widetilde{\ell}^{(\bar{r})}$ and the generic case holds for $(\nu, J)^{(\bar{r})}$. It follows that for all $k \geq \bar{r}, \widetilde{\bar{\ell}}^{(k)}=\widetilde{\ell}^{(k)}$ and $\overline{\tilde{\ell}}^{(k)}=\bar{\ell}^{(k)}$. Therefore $\widetilde{\bar{r}}=\widetilde{r}$ and $\overline{\widetilde{r}} \leq \bar{r}$. Suppose $\overline{\widetilde{r}}<\bar{r}$. Then $\overline{\left.\bar{\ell}^{(\bar{r}}\right)}=\infty>\bar{\ell}^{(\overline{\widetilde{r}})}$. It follows that Case 3 holds for $(\nu, J)^{(\overline{\widetilde{r}})}$. But then $\widetilde{\bar{\ell}}^{(\overline{\widetilde{r}})}=\overline{\left.\bar{\ell}^{(\bar{r}}\right)}=\infty$ so that $\widetilde{r} \leq \overline{\widetilde{r}}<\bar{r}<\widetilde{r}$, which is a contradiction. Therefore $\overline{\widetilde{r}}=\bar{r}$ and $\widetilde{\bar{r}}=\widetilde{r}$ as desired.

If $\bar{r}>\widetilde{r}$ a similar proof shows $\bar{r}=\bar{r}$ and $\widetilde{r}=\widetilde{r}$. So it may be assumed that $r=\widetilde{r}=\bar{r}$. Notice that $\widetilde{\ell}^{(r)}=\bar{\ell}^{(r)}=\infty$ implies that $\widetilde{\bar{\ell}}^{(r)}=\overline{\tilde{\ell}}^{(r)}=\infty$ so that $\widetilde{r} \leq r$ and $\overline{\widetilde{r}} \leq r$. Suppose Case 3 does not hold for $(\nu, J)^{(r-1)}$. Then $\widetilde{\bar{\ell}}^{(r-1)} \leq \widetilde{\ell}^{(r-1)}<\infty$ and $\overline{\tilde{\ell}}^{(r-1)} \leq \bar{\ell}^{(r-1)}<\infty$ so that $\widetilde{\bar{r}} \geq r$ and $\overline{\widetilde{r}} \geq r$. Thus $\overline{\widetilde{r}}=\widetilde{r}=r$ as desired. Otherwise suppose Case 3 holds for $(\nu, J)^{(r-1)}$. Recall that in Case 3 one has $\widetilde{\bar{\ell}}^{(r-1)}=\overline{\bar{\ell}}^{(r-1)}$. If $\widetilde{\bar{\ell}}^{(r-1)}<\infty$ then $\widetilde{\bar{r}}=\overline{\widetilde{r}}=r$ as desired. Otherwise $\widetilde{\bar{\ell}}^{(r-1)}=\overline{\bar{\ell}}^{(r-1)}=\infty$ so that $\widetilde{\bar{r}} \leq r-1$, $\overline{\widetilde{r}} \leq r-1$ and furthermore $\lambda_{r-1}^{t}=\lambda_{r}^{t}$. Suppose that $\widetilde{\bar{\ell}}^{(r-2)}=\infty$. Then it is clear from Lemma A.2 that either $\widetilde{\ell}^{(r-2)}=\infty$ or $\widetilde{\ell}^{(r-1)}=\infty$ contradicting the definition of $r$. Similarly $\overline{\tilde{\ell}}^{(r-2)}=\infty$ leads to a contradiction. Hence $\widetilde{\bar{r}}=\overline{\widetilde{r}}=r-1$ as desired.

The rest of this section is devoted to the proofs of Lemmas A.2 and A.3.

Proof of Lemma A.A. The proof proceeds by induction on $k$. There is nothing to prove unless at least one of $\bar{\ell}^{(k)}$ and $\widetilde{\ell}^{(k)}$ is finite. If one is finite and the other infinite then obviously $\bar{\delta}$ and $\widetilde{\delta}$ choose different strings and $\widetilde{\bar{\ell}}^{(k)}=\widetilde{\ell}^{(k)}$ and $\overline{\widetilde{\ell}}^{(k)}=\bar{\ell}^{(k)}$. So it is assumed that both $\bar{\ell}^{(k)}$ and $\widetilde{\ell}^{(k)}$ are finite.

For the base case $k=0$, observe that the doubly singular case holds precisely when $R=\left(\left(1^{\mu_{1}}\right)\right)$. Then $\operatorname{RC}\left(\lambda^{t} ; R^{t}\right)$ is empty unless $\lambda=\left(1^{\mu_{1}}\right)$, and in that case consists of the empty rigged configuration $(\emptyset, \emptyset)$. Then $\bar{\ell}^{(0)}=\widetilde{\ell}^{(0)}=\mu_{1}$ and $\widetilde{\bar{\ell}}^{(0)}=\overline{\tilde{\ell}}^{(0)}=\mu_{1}-1$. But $\overline{\operatorname{rk}}(\emptyset, \emptyset)=\widetilde{\operatorname{rk}}(\emptyset, \emptyset)=1$ so 
this case is never used in the inductive step. In the generic case $L \geq 2$, $\widetilde{\bar{\ell}}^{(0)}=\widetilde{\ell}^{(0)}=\mu_{1}$ and $\overline{\tilde{\ell}}^{(0)}=\bar{\ell}^{(0)}=\mu_{L}$.

Now assume $k \geq 1$. Note that

$$
\begin{aligned}
& \bar{\ell}^{(k)} \geq \overline{\bar{\ell}}^{(k-1)} \\
& \widetilde{\ell}^{(k)} \geq \widetilde{\bar{\ell}}^{(k-1)} .
\end{aligned}
$$

If the generic case, Case 1 or Case 2 occurs at $k-1$, this follows immediately from $\bar{\ell}^{(k)} \geq \bar{\ell}^{(k-1)} \geq \overline{\bar{\ell}}^{(k-1)}$ and $\widetilde{\ell}^{(k)} \geq \widetilde{\ell}^{(k-1)} \geq \widetilde{\bar{\ell}}^{(k-1)}$. For Case 3 at $k-1$ (A.1) holds by induction hypothesis.

Generic case. Observe that $\widetilde{\ell}^{(k)}=\widetilde{\bar{\ell}}^{(k)}$ is obtained from $\bar{\ell}^{(k)}=\overline{\tilde{\ell}}^{(k)}$ under conjugation by the involution $\theta_{R}^{\text {ev }}$, so we shall only prove the latter. By Remark A.1, the singular string in $\nu^{(k)}$ of length $\bar{\ell}^{(k)}$ remains singular in passing to $\widetilde{\nu}^{(k)}$. Since $\overline{\tilde{\ell}}^{(k-1)} \leq \bar{\ell}^{(k)}$ by (A.1), it follows that $\overline{\tilde{\ell}}^{(k)} \leq \bar{\ell}^{(k)}$.

If $\overline{\tilde{\ell}}^{(k)}=\bar{\ell}^{(k)}$ we are done. By induction hypothesis $\overline{\tilde{\ell}}^{(k)} \geq \overline{\tilde{\ell}}^{(k-1)} \geq$ $\bar{\ell}^{(k-1)}-1$. Let us first assume that $\bar{\ell}^{(k-1)} \leq \overline{\tilde{\ell}}^{(k)}<\bar{\ell}^{(k)}$. By Remark A.1 this is only possible if the string selected by $\bar{\delta}$ acting on $\widetilde{\nu}^{(k)}$ is the string shortened by $\widetilde{\delta}$ acting on $\nu^{(k)}$. This string in $\widetilde{\nu}^{(k)}$ has length $\widetilde{\ell}^{(k)}-1$ and label 0 . We show that this cannot occur. For this it is enough to show that

$$
P_{\widetilde{\ell}^{(k)}-1}^{(k)}(\widetilde{\nu})>0 \quad \text { if } \quad \bar{\ell}^{(k-1)}<\widetilde{\ell}^{(k)} \leq \bar{\ell}^{(k)} \text { and } \quad \overline{\tilde{\ell}}^{(k-1)}<\widetilde{\ell}^{(k)} .
$$

Otherwise we may assume that $\bar{\ell}^{(k-1)}-1=\overline{\bar{\ell}}^{(k)}<\bar{\ell}^{(k)}$. This means that Case 3 occurs at $k-1$ so that $m_{\bar{\ell}^{(k-1)}-1}\left(\nu^{(k)}\right)=0$ and $\widetilde{\ell}^{(k-1)}=\bar{\ell}^{(k-1)}$. Hence $\overline{\tilde{\ell}}^{(k)}=\bar{\ell}^{(k-1)}-1$ can only occur if $\widetilde{\ell}^{(k)}=\widetilde{\ell}^{(k-1)}=\bar{\ell}^{(k-1)}$. To prove that this cannot happen it suffices to show that

$$
P_{\widetilde{\ell}^{(k)}-1}^{(k)}(\widetilde{\nu})>0 \quad \text { if } m_{\widetilde{\ell}^{(k-1)}-1}\left(\nu^{(k)}\right)=0 \text { and } \bar{\ell}^{(k-1)}=\widetilde{\ell}^{(k-1)}=\widetilde{\ell}^{(k)} \leq \bar{\ell}^{(k)} .
$$

By $(3.16)$ with $n=\widetilde{\ell}^{(k)}-1$,

$$
P_{\widetilde{\ell}^{(k)}-1}^{(k)}(\widetilde{\nu})=P_{\widetilde{\ell}^{(k)}-1}^{(k)}(\nu)-\chi\left(\widetilde{\ell}^{(k-1)} \leq \widetilde{\ell}^{(k)}-1\right) .
$$

We proceed by considering various cases that exhaust all possibilities, and show that (A.2) and (A.3) both hold in each case.

First assume that $m_{\tilde{\ell}^{(k)}-1}\left(\nu^{(k)}\right)=0$ and $\widetilde{\ell}^{(k-1)}=\widetilde{\ell}^{(k)}$. Since $\bar{\ell}^{(k-1)} \leq$ $\widetilde{\ell}^{(k)} \leq \bar{\ell}^{(k)}$ by assumption, $P_{\widetilde{\ell}^{(k)}}^{(k)}(\nu) \geq 1$, for otherwise $\bar{\ell}^{(k)}=\widetilde{\ell}^{(k)}$ and $\bar{\delta}$ and $\widetilde{\delta}$ would select the same string in $\nu^{(k)}$. By Lemma 3.11 with $n=\widetilde{\ell}^{(k)}-1$ 
it follows that $P_{\widetilde{\ell}^{(k)}-1}^{(k)}(\nu) \geq 1$, which by $($ A.4 $)$ implies $P_{\widetilde{\ell}^{(k)}-1}^{(k)}(\widetilde{\nu}) \geq 1$. This proves in particular (A.3).

For all remaining cases (A.3) holds vacuously. Hence it may be assumed that the hypotheses of (A.2) hold.

Assume that $m_{\widetilde{\ell}^{(k)}-1}\left(\nu^{(k)}\right)>0$ and $\widetilde{\ell}^{(k-1)}<\widetilde{\ell}^{(k)}$. Since $\widetilde{\ell}^{(k-1)} \leq \widetilde{\ell}^{(k)}-1<$ $\widetilde{\ell}^{(k)}$ and $\bar{\ell}^{(k-1)} \leq \widetilde{\ell}^{(k)}-1<\bar{\ell}^{(k)}$, there cannot be strings in $\nu^{(k)}$ of length $\widetilde{\ell}^{(k)}-1$ that have label zero or are singular. Since there are strings in $\nu^{(k)}$ of length $\widetilde{\ell}^{(k)}-1$, there must be an available label that is neither zero nor maximum. Thus $P_{\widetilde{\ell}^{(k)}-1}^{(k)}(\nu) \geq 2$, and by $($ A.4 $), P_{\widetilde{\ell}^{(k)}-1}^{(k)}(\widetilde{\nu}) \geq 1$.

Now assume that $m_{\widetilde{\ell}^{(k)}-1}\left(\nu^{(k)}\right)>0$ and $\widetilde{\ell}^{(k-1)}=\widetilde{\ell}^{(k)}$. Since $\bar{\ell}^{(k-1)}<$ $\widetilde{\ell}^{(k)} \leq \bar{\ell}^{(k)}$ there cannot be a singular string of length $\widetilde{\ell}^{(k)}-1$ in $\nu^{(k)}$. Hence $P_{\widetilde{\ell}^{(k)}-1}^{(k)}(\nu) \geq 1$ and by $($ A.4 $) P_{\widetilde{\ell}^{(k)}-1}^{(k)}(\widetilde{\nu}) \geq 1$.

Finally consider the case $m_{\widetilde{\ell}^{(k)}-1}\left(\nu^{(k)}\right)=0$ and $\widetilde{\ell}^{(k-1)}<\widetilde{\ell}^{(k)}$. By A.4 if $P_{\widetilde{\ell}^{(k)}-1}^{(k)}(\nu) \geq 2$ then we are done, so it may be assumed that $P_{\widetilde{\ell}^{(k)}-1}^{(k)}(\nu)=1$. Let $\ell$ be maximal such that $\ell<\widetilde{\ell}^{(k)}$ and $m_{\ell}\left(\nu^{(k)}\right) \geq 1$. If no such $\ell$ exists set $\ell=0$.

Suppose that $P_{\widetilde{\ell}^{(k)}}^{(k)}(\nu)=0$. By the definition of $\widetilde{\ell}^{(k)}, m_{\widetilde{\ell}^{(k)}}\left(\nu^{(k)}\right) \geq 1$. Hence there is a string of length $\widetilde{\ell}^{(k)}$ in $\nu^{(k)}$, which is singular since its vacancy number is zero. Due to the assumption that $\bar{\ell}^{(k-1)}<\widetilde{\ell}^{(k)} \leq \bar{\ell}^{(k)}$, the definition of $\bar{\ell}^{(k)}$ yields $\bar{\ell}^{(k)}=\widetilde{\ell}^{(k)}$. This means that the doubly singular case holds for $\nu^{(k)}$, which is a contradiction.

Suppose $P_{\widetilde{\ell}^{(k)}}^{(k)}(\nu)=1$. By Lemma 3.11 we have $P_{n}^{(k)}(\nu)=1$ for $\ell<n \leq$ $\widetilde{\ell}^{(k)}$ and $0 \leq P_{\ell}^{(k)}(\nu) \leq 1$. By $(3.10), m_{n}\left(\nu^{(k-1)}\right)=0$ for $\ell+1<n<\widetilde{\ell}^{(k)}$ and $0 \leq m_{\ell+1}\left(\nu^{(k-1)}\right) \leq 1$.

First consider $m_{\ell+1}\left(\nu^{(k-1)}\right)=0$. If $\ell=0$ there is no string of length smaller than $\widetilde{\ell}^{(k)}$ in $\nu^{(k-1)}$ so that $\widetilde{\ell}^{(k-1)} \geq \widetilde{\ell}^{(k)}$. This contradicts the assumptions. So assume that $\ell>0$. Since $\widetilde{\ell}^{(k-1)} \leq \ell$ and $\bar{\ell}^{(k-1)} \leq \ell$ this requires that the strings of length $\ell$ in $\nu^{(k)}$ are neither singular nor have label zero so that $P_{\ell}^{(k)}(\nu) \geq 2$. This is a contradiction.

Hence assume that $m_{\ell+1}\left(\nu^{(k-1)}\right)=1$. By $(3.10)$ at $n=\ell+1$ this implies that $P_{\ell}^{(k)}(\nu)=0$. Since $\widetilde{\ell}^{(k)}>\ell$ and $\bar{\ell}^{(k)}>\ell$ this requires that $\widetilde{\ell}^{(k-1)}=$ $\bar{\ell}^{(k-1)}=\ell+1$ and hence $P_{\ell+1}^{(k-1)}(\nu)=0$. Since $m_{\ell+1}\left(\nu^{(k-1)}\right)=1$, Case 1 or Case 3 occurs at $k-1$. If Case 3 occurs, $\widetilde{\bar{\ell}}^{(k-1)}=\overline{\bar{\ell}}^{(k-1)} \geq \widetilde{\ell}^{(k)}$ which contradicts the assumptions. Therefore $\nu^{(k-1)}$ must be in Case 1 and $\ell>0$. By induction $m_{\ell}\left(\nu^{(k)}\right)=0$ which contradicts the definition of $\ell$.

Now suppose $P_{\widetilde{\ell}^{(k)}}^{(k)}(\nu)=2$. Again (3.10) and Lemma 3.11 fail unless $\widetilde{\ell}^{(k)}=$ $\ell+2$ and $P_{\ell}^{(k)}(\nu)=0$. By $(3.10)$ with $n=\ell+1=\widetilde{\ell}^{(k)}-1, m_{\ell+1}\left(\nu^{(k-1)}\right)=0$. 
If $\ell=0$ then $\widetilde{\ell}^{(k-1)}<\widetilde{\ell}^{(k)}=2$ which forces $\widetilde{\ell}^{(k-1)}=1$, but there is no string of length 1 in $\nu^{(k-1)}$, which is a contradiction. So suppose $\ell>0$. Since $\bar{\ell}^{(k-1)}<\widetilde{\ell}^{(k)}, \widetilde{\ell}^{(k-1)}<\widetilde{\ell}^{(k)}$ and $m_{\tilde{\ell}^{(k)}-1}\left(\nu^{(k-1)}\right)=0$, one finds $\bar{\ell}^{(k-1)} \leq \ell$ and $\tilde{\ell}^{(k-1)} \leq \ell$. Also there is a string of length $\ell$ in $\nu^{(k)}$, which is both singular and has label zero since $P_{\ell}^{(k)}(\nu)=0$. But $\ell<\widetilde{\ell}^{(k)} \leq \bar{\ell}^{(k)}$, which contradicts the definition of $\bar{\ell}^{(k)}$ and $\widetilde{\ell}^{(k)}$.

If $P_{\widetilde{\ell}^{(k)}}^{(k)}(\nu)>2$ then there is an immediate contradiction since (3.11) fails for $n=\widetilde{\ell}^{(k)}-1$.

This completes the proof of $(\sqrt{\mathrm{A.2}})$ and $(\mathrm{A.3})$ and hence the proof of the generic case for $\nu^{(k)}$.

Doubly singular case. Since there is a string of length $\ell$ in $\nu^{(k)}$ that is both singular and has label zero, it must have vacancy number zero, that is, $P_{\ell}^{(k)}(\nu)=0$.

Case 1: $\widetilde{\bar{\ell}}^{(k)}<\ell$. By induction we have $\widetilde{\bar{\ell}}^{(k)} \geq \widetilde{\bar{\ell}}^{(k-1)} \geq \widetilde{\ell}^{(k-1)}-1$. First assume that $\widetilde{\ell}^{(k-1)} \leq \widetilde{\bar{\ell}}^{(k)}<\ell$. In the light of Remark A.1, $\widetilde{\delta}$ must select the string in $\bar{\nu}^{(k)}$ that was shortened by $\bar{\delta}$ in the transformation $(\nu, J) \mapsto$ $(\bar{\nu}, \overline{\mathrm{J}})$, so that $\widetilde{\bar{\ell}}^{(k)}=\ell-1$. This string in $\bar{\nu}^{(k)}$ is singular since it was shortened by $\bar{\delta}$ and has label zero since it is selected by $\widetilde{\delta}$, so $P_{\ell-1}^{(k)}(\bar{\nu})=0$. The case $\widetilde{\ell}^{(k-1)}-1=\widetilde{\bar{\ell}}^{(k)}<\ell$ can only occur for Case 1 at $k-1$. By induction this implies that $m_{\widetilde{\ell}^{(k-1)}-1}\left(\nu^{(k)}\right)=0$. For $\widetilde{\ell}^{(k-1)}-1=\widetilde{\bar{\ell}}^{(k)}$ one needs $m_{\widetilde{\ell}^{(k-1)-1}}\left(\bar{\nu}^{(k)}\right)>0$ so that $\ell=\widetilde{\ell}^{(k-1)}$. Hence $\widetilde{\bar{\ell}}^{(k)}=\ell-1$ and $P_{\ell-1}^{(k)}(\bar{\nu})=0$ as before.

The goal is to show that $\overline{\bar{\ell}}^{(k)}=\ell-1$. Since $\widetilde{\ell}^{(k)}=\ell$, it follows that $m_{\ell-1}\left(\widetilde{\nu}^{(k)}\right) \geq 1$. It suffices to show that $\overline{\tilde{\ell}}^{(k-1)} \leq \ell-1$ and $P_{\ell-1}^{(k)}(\widetilde{\nu})=0$. For then $\overline{\bar{\ell}}^{(k)}<\ell$, and by the same arguments as before it follows that $\overline{\bar{\ell}}^{(k)}=\ell-1$.

By $(3.9),(3.16)$, and $P_{\ell-1}^{(k)}(\bar{\nu})=0$,

$$
\begin{aligned}
P_{\ell-1}^{(k)}(\nu) & =P_{\ell-1}^{(k)}(\widetilde{\nu})+\chi\left(\widetilde{\ell}^{(k-1)} \leq \ell-1\right) \\
& =\chi\left(\bar{\ell}^{(k-1)} \leq \ell-1\right) .
\end{aligned}
$$

Suppose that $\overline{\tilde{\ell}}^{(k-1)} \geq \ell$. Now $\widetilde{\bar{\ell}}^{(k-1)} \leq \widetilde{\bar{\ell}}^{(k)}=\ell-1$ so $\widetilde{\bar{\ell}}^{(k-1)} \neq \overline{\bar{\ell}}^{(k-1)}$. By induction, the generic case holds for $\nu^{(k-1)}$, and $\widetilde{\bar{\ell}}^{(k-1)}=\widetilde{\ell}^{(k-1)}$ and $\overline{\tilde{\ell}}^{(k-1)}=\bar{\ell}^{(k-1)}$. So $\bar{\ell}^{(k-1)}=\overline{\tilde{\ell}}^{(k-1)} \geq \ell$ and $\widetilde{\ell}^{(k-1)}=\widetilde{\bar{\ell}}^{(k-1)} \leq \ell-1$. This leads to a contradiction in evaluating (A.5), so $\overline{\bar{\ell}}^{(k-1)} \leq \ell-1$. 
Suppose $P_{\ell-1}^{(k)}(\widetilde{\nu}) \geq 1$. Then by (A.5), $\widetilde{\ell}^{(k-1)} \geq \ell$ and $\bar{\ell}^{(k-1)} \leq \ell-1$. Since $\widetilde{\ell}^{(k-1)} \neq \bar{\ell}^{(k-1)}$, by induction the generic case holds for $\nu^{(k-1)}$ and $\widetilde{\bar{\ell}}^{(k-1)}=\widetilde{\ell}^{(k-1)} \geq \ell$, which contradicts $\ell>\widetilde{\bar{\ell}}^{(k)} \geq \widetilde{\bar{\ell}}^{(k-1)}$.

Therefore $P_{\ell-1}^{(k)}(\widetilde{\nu})=0$ and $\overline{\tilde{\ell}}^{(k)}=\widetilde{\bar{\ell}}^{(k)}=\ell-1$.

To finish Case 1 it suffices to show that $m_{\ell-1}\left(\nu^{(k+1)}\right)=0$.

Since it has been shown that $P_{\ell-1}^{(k)}(\widetilde{\nu})=0$, A.5 becomes

$$
P_{\ell-1}^{(k)}(\nu)=\chi\left(\widetilde{\ell}^{(k-1)} \leq \ell-1\right)=\chi\left(\bar{\ell}^{(k-1)} \leq \ell-1\right) .
$$

Suppose that $P_{\ell-1}^{(k)}(\nu)=0$. By A.6), $\widetilde{\ell}^{(k-1)}=\ell$ and $\bar{\ell}^{(k-1)}=\ell$. Now $\widetilde{\bar{\ell}}^{(k-1)} \leq \widetilde{\bar{\ell}}^{(k)}=\ell-1<\widetilde{\ell}^{(k-1)}$, so Case 1 holds for $\nu^{(k-1)}$. By induction $\widetilde{\bar{\ell}}^{(k-1)}=\widetilde{\ell}^{(k-1)}-1$ and $m_{\widetilde{\ell}^{(k-1)}-1}\left(\nu^{(k)}\right)=0$, that is, $m_{\ell-1}\left(\nu^{(k)}\right)=0$. By (3.10) for $n=\ell-1$, it follows that $m_{\ell-1}\left(\nu^{(k+1)}\right)=0$, as desired.

Otherwise $P_{\ell-1}^{(k)}(\nu)=1$. Here $\ell \geq 2$. By A.6), $\widetilde{\ell}^{(k-1)} \leq \ell-1$ and $\bar{\ell}^{(k-1)} \leq \ell-1$. By the minimality of $\widetilde{\ell}^{(k)}$ and $\bar{\ell}^{(k)}$, there cannot be strings in $\nu^{(k)}$ of length $\ell-1$ that are singular or have label zero, so $m_{\ell-1}\left(\nu^{(k)}\right)=0$. Applying (3.10) at $n=\ell-1$ and using the fact that $P_{\ell}^{(k)}(\nu)=0$ (since the doubly singular case holds for $\nu^{(k)}$ ) one obtains

$$
P_{\ell-2}^{(k)}(\nu)+m_{\ell-1}\left(\nu^{(k-1)}\right)+m_{\ell-1}\left(\nu^{(k+1)}\right) \leq 2 .
$$

If $m_{\ell-1}\left(\nu^{(k-1)}\right)=2$ then by $\left(\right.$ A.7) $m_{\ell-1}\left(\nu^{(k+1)}\right)=0$ as desired.

Suppose $m_{\ell-1}\left(\nu^{(k-1)}\right)=1$. If $P_{\ell-2}^{(k)}(\nu)=1$ then again we conclude $m_{\ell-1}\left(\nu^{(k+1)}\right)=0$ by (A.7). So assume that $P_{\ell-2}^{(k)}(\nu)=0$. If $m_{\ell-2}\left(\nu^{(k)}\right)=0$ then by (3.10) with $n=\ell-2, P_{\ell-1}^{(k)}(\nu)=0$ which is a contradiction. Otherwise $m_{\ell-2}\left(\nu^{(k)}\right) \geq 1$. Then there is a singular string of length $\ell-2$ in $\nu^{(k)}$. By the definition of $\bar{\ell}^{(k)}$ we have $\bar{\ell}^{(k-1)}>\ell-2$, that is, $\bar{\ell}^{(k-1)}=\ell-1$. Similarly $\widetilde{\ell}^{(k-1)}=\ell-1$. Since $m_{\ell-1}\left(\nu^{(k-1)}\right)=1$, by induction the doubly singular case holds for $\nu^{(k-1)}$. Now $\widetilde{\bar{\ell}}^{(k-1)} \leq \widetilde{\bar{\ell}}^{(k)}=\ell-1=\widetilde{\ell}^{(k-1)}$, so Case 3 is impossible. Since $m_{\ell-1}\left(\nu^{(k-1)}\right)=1$, Case 2 is also impossible. So Case 1 holds for $\nu^{(k-1)}$. It follows that $m_{\widetilde{\ell}^{(k-1)}-1}\left(\nu^{(k)}\right)=0$, that is, $m_{\ell-2}\left(\nu^{(k)}\right)=0$. But this is a contradiction.

Suppose that $m_{\ell-1}\left(\nu^{(k-1)}\right)=0$. Then $\bar{\ell}^{(k-1)} \leq \ell-2$ and $\widetilde{\ell}^{(k-1)} \leq \ell-2$. This yields a contradiction unless $\ell>2$. By the minimality of $\bar{\ell}^{(k)}$ and $\widetilde{\ell}^{(k)}$, there cannot be strings in $\nu^{(k)}$ of length $\ell-2$ that are either singular or have label zero, so it follows that either $m_{\ell-2}\left(\nu^{(k)}\right)=0$ or $P_{\ell-2}^{(k)}(\nu) \geq 2$. Using (A.7) the latter immediately yields the desired result $m_{\ell-1}\left(\nu^{(k+1)}\right)=0$, so assume that $m_{\ell-2}\left(\nu^{(k)}\right)=0$ and $P_{\ell-2}^{(k)}(\nu) \leq 1$. 
If $P_{\ell-2}^{(k)}(\nu)=0$, by 3.10 with $n=\ell-2$ it follows that $P_{\ell-1}^{(k)}(\nu)=0$, which is a contradiction.

So $P_{\ell-2}^{(k)}(\nu)=1$.

Let $p<\ell-1$ be maximal such that $m_{p}\left(\nu^{(k)}\right) \geq 1$; if no such $p$ exists set $p=0$. Then by Lemma 3.11, $P_{n}^{(k)}(\nu)=1$ for $p<n<\ell$ and $P_{p}^{(k)}(\nu) \leq$ 1. By (3.10) it follows that $m_{n}\left(\nu^{(k-1)}\right)=0$ for $p+1<n<\ell-1$ and $m_{\ell-1}\left(\nu^{(k-1)}\right)+m_{\ell-1}\left(\nu^{(k+1)}\right) \leq 1$. If $m_{\ell-1}\left(\nu^{(k-1)}\right)=1$ then $m_{\ell-1}\left(\nu^{(k+1)}\right)=0$ as desired. Hence assume that $m_{\ell-1}\left(\nu^{(k-1)}\right)=0$.

Suppose $P_{p}^{(k)}(\nu)=1$ which implies $p>0$. Then by $(3.10)$ it follows that $m_{p+1}\left(\nu^{(k-1)}\right)=0$. Since $\widetilde{\ell}^{(k-1)}<\ell-1$ and $\bar{\ell}^{(k-1)}<\ell-1$, it follows that $\tilde{\ell}^{(k-1)} \leq p$ and $\bar{\ell}^{(k-1)} \leq p$. Also $m_{p}\left(\nu^{(k)}\right) \geq 1$ since $p>0$. Then there is a singular string of length $p$ in $\nu^{(k)}$ or one of label zero, contradicting the definition of $\bar{\ell}^{(k)}$ or $\widetilde{\ell}^{(k)}$.

Therefore $P_{p}^{(k)}(\nu)=0$. By 3.10$)$ for $n=p+1$, we have $m_{p+1}\left(\nu^{(k-1)}\right)+$ $m_{p+1}\left(\nu^{(k+1)}\right) \leq 1$.

Suppose that $m_{p+1}\left(\nu^{(k-1)}\right)=0$. If $p>0$ then the proof proceeds as before. If $p=0$ then there are no strings in $\nu^{(k-1)}$ of length less than $\ell$. This contradicts $\widetilde{\ell}^{(k-1)} \leq \ell-1$.

So assume that $m_{p+1}\left(\nu^{(k-1)}\right)=1$.

Certainly $\tilde{\ell}^{(k-1)} \leq p+1$ and $\bar{\ell}^{(k-1)} \leq p+1$. If either $\tilde{\ell}^{(k-1)} \leq p$ or $\bar{\ell}^{(k-1)} \leq p$ then there is a string in $\nu^{(k)}$ of length $p$ that (due to $P_{p}^{(k)}(\nu)=0$ ) is either singular or has label zero. But $p<\ell$, contradicting the definition of $\bar{\ell}^{(k)}$ or $\widetilde{\ell}^{(k)}$.

So $\widetilde{\ell}^{(k-1)}=p+1$ and $\bar{\ell}^{(k-1)}=p+1$. Since $m_{p+1}\left(\nu^{(k-1)}\right)=1, m_{n}\left(\widetilde{\nu}^{(k-1)}\right)=$ 0 for $p<n<\ell$.

Now $\overline{\widetilde{\ell}}^{(k-1)} \leq \overline{\widetilde{\ell}}^{(k)}=\ell-1$, so $\overline{\widetilde{\ell}}^{(k-1)} \leq p<p+1=\bar{\ell}^{(k-1)}$. So Case 1 holds for $\nu^{(k-1)}$. By induction $\overline{\tilde{\ell}}^{(k-1)}=\bar{\ell}^{(k-1)}-1=p$ and $m_{p}\left(\nu^{(k)}\right)=0$ which contradicts the construction of $p$.

This concludes the proof of Case 1 .

Using the involution $\theta_{R}^{\text {ev }}$, the above argument also shows that if $\overline{\tilde{\ell}}^{(k)}<\ell$ then $\overline{\tilde{\ell}}^{(k)}=\widetilde{\bar{\ell}}^{(k)}=\ell-1$.

Case 2: $\widetilde{\bar{\ell}}^{(k)}=\ell$. It will be shown that $\overline{\tilde{\ell}}^{(k)}=\ell$. By Case $1, \overline{\bar{\ell}}^{(k)} \geq \ell$.

The assumption $\widetilde{\bar{\ell}}^{(k)}=\ell$ implies that $m_{\ell}\left(\bar{\nu}^{(k)}\right) \geq 1$. Since $\bar{\ell}^{(k)}=\ell$ one part of size $\ell$ is shortened in passing from $\nu^{(k)}$ to $\bar{\nu}^{(k)}$, so that $m_{\ell}\left(\nu^{(k)}\right) \geq 2$. Now $P_{\ell}^{(k)}(\nu)=0$, so there is at least one singular string in $\nu^{(k)}$ that is not selected by $\widetilde{\delta}$ acting on $(\nu, J)$. By Remark A.1 this string remains singular of length $\ell$ in passing to $\widetilde{\nu}^{(k)}$. This shows that there is a singular string of length $\ell$ in $\widetilde{\nu}^{(k)}$. Thus to prove $\overline{\tilde{\ell}}^{(k)}=\ell$ it suffices to show that $\overline{\tilde{\ell}}^{(k-1)} \leq \ell$. 
If $\overline{\tilde{\ell}}^{(k-1)} \leq \bar{\ell}^{(k-1)}$, then $\overline{\tilde{\ell}}^{(k-1)} \leq \bar{\ell}^{(k-1)} \leq \bar{\ell}^{(k)}=\ell$. Otherwise $\overline{\tilde{\ell}}^{(k-1)}>$ $\bar{\ell}^{(k-1)}$, so Case 3 holds for $\nu^{(k-1)}$, and by induction $\overline{\tilde{\ell}}^{(k-1)}=\widetilde{\bar{\ell}}^{(k-1)} \leq \widetilde{\bar{\ell}}^{(k)}=\ell$.

By applying $\theta_{R}^{\text {ev }}$ this also shows that if $\overline{\tilde{\ell}}^{(k)}=\ell$ then $\widetilde{\bar{\ell}}^{(k)}=\ell$.

Case 3: $\widetilde{\bar{\ell}}^{(k)}>\ell$. By Cases 1 and 2 it follows that $\overline{\tilde{\ell}}^{(k)}>\ell$. It will be shown that $\overline{\widetilde{\ell}}^{(k)}=\widetilde{\bar{\ell}}^{(k)}$ and that

$$
\begin{aligned}
P_{\ell+1}^{(k)}(\nu) & =0 \\
P_{\ell-1}^{(k)}(\nu) & =2-m_{\ell}\left(\nu^{(k-1)}\right) \\
m_{\ell}\left(\nu^{(k+1)}\right) & =0 .
\end{aligned}
$$

Suppose $m_{\ell}\left(\nu^{(k)}\right) \geq 2$. Since $P_{\ell}^{(k)}(\nu)=0$, there is a string in $\nu^{(k)}$ of length $\ell$ and label zero that is not selected by $\bar{\delta}$ in passing to $\bar{\nu}^{(k)}$. By Remark A.1 it follows that there is a string in $\bar{\nu}^{(k)}$ of length $\ell$ with label zero. Since $\widetilde{\bar{\ell}}^{(k)}>\ell$, this string cannot be selected, that is, $\widetilde{\bar{\ell}}^{(k-1)}>\ell$. Now $\widetilde{\ell}^{(k-1)} \leq \widetilde{\ell}^{(k)}=\ell<\widetilde{\bar{\ell}}^{(k-1)}$. By induction Case 3 holds at $k-1$. This implies in particular $\widetilde{\ell}^{(k)} \geq \widetilde{\bar{\ell}}^{(k-1)}>\ell$, which is a contradiction.

Therefore $m_{\ell}\left(\nu^{(k)}\right)=1$. By (3.10) with $n=\ell, P_{\ell}^{(k)}(\nu)=0$, and $m_{\ell}\left(\nu^{(k)}\right)=1$, we have

$$
P_{\ell-1}^{(k)}(\nu)+P_{\ell+1}^{(k)}(\nu)+m_{\ell}\left(\nu^{(k-1)}\right)+m_{\ell}\left(\nu^{(k+1)}\right) \leq 2 .
$$

We distinguish the three cases $m_{\ell}\left(\nu^{(k-1)}\right) \in\{0,1,2\}$.

We start with $m_{\ell}\left(\nu^{(k-1)}\right)=0$. Recall that $\bar{\ell}^{(k-1)} \leq \bar{\ell}^{(k)}=\ell$ and $\widetilde{\ell}^{(k-1)} \leq$ $\tilde{\ell}^{(k)}=\ell$. However the inequalities must be strict since there are no strings of length $\ell$ in $\nu^{(k-1)}$. So

$$
\begin{aligned}
& \bar{\ell}^{(k-1)} \leq \ell-1 \\
& \tilde{\ell}^{(k-1)} \leq \ell-1 .
\end{aligned}
$$

If $m_{\ell-1}\left(\nu^{(k)}\right)>0$ then necessarily $P_{\ell-1}^{(k)}(\nu) \geq 2$ since otherwise a string in $\nu^{(k)}$ of length $\ell-1$ is selected by $\bar{\delta}$ or $\widetilde{\delta}$, contradicting $\bar{\ell}^{(k)}=\widetilde{\ell}^{(k)}=\ell$. By (A.11) we conclude that $P_{\ell-1}^{(k)}(\nu)=2, P_{\ell+1}^{(k)}(\nu)=0$, and $m_{\ell}\left(\nu^{(k+1)}\right)=0$.

Now assume $m_{\ell-1}\left(\nu^{(k)}\right)=0$. By (3.9) with $n=\ell-1, \bar{\ell}^{(k-1)} \leq \ell-1$, and $\bar{\ell}^{(k)}=\ell$, we have

$$
P_{\ell-1}^{(k)}(\nu)=P_{\ell-1}^{(k)}(\bar{\nu})+1 .
$$

Suppose that $P_{\ell-1}^{(k)}(\bar{\nu})=0$. Since $m_{\ell-1}\left(\bar{\nu}^{(k)}\right)=1$ there is a string of length $\ell-1$ and label zero in $\bar{\nu}^{(k)}$. But $\widetilde{\bar{\ell}}^{(k)}>\ell$, so the only way that this string is not selected is if $\widetilde{\bar{\ell}}^{(k-1)}>\ell-1$. But by (A.12) $\ell-1 \geq \widetilde{\ell}^{(k-1)}$, so 
$\widetilde{\bar{\ell}}^{(k-1)}>\widetilde{\ell}^{(k-1)}$. This is Case 3 for $\nu^{(k-1)}$. By induction $\widetilde{\bar{\ell}}^{(k-1)}=\overline{\tilde{\ell}}^{(k-1)}>$ $\widetilde{\ell}^{(k-1)}=\bar{\ell}^{(k-1)}$ and $\widetilde{\ell}^{(k)} \geq \widetilde{\bar{\ell}}^{(k-1)}$. But $\widetilde{\ell}^{(k)}=\ell$ and $\widetilde{\bar{\ell}}^{(k-1)} \geq \ell$ so $\widetilde{\bar{\ell}}^{(k-1)}=\ell$. Then $m_{\ell}\left(\bar{\nu}^{(k-1)}\right) \geq 1$. However $\bar{\ell}^{(k-1)} \leq \ell-1$ by $\left(\right.$ A.12 so $m_{\ell}\left(\nu^{(k-1)}\right) \geq 1$, which is a contradiction.

Therefore $P_{\ell-1}^{(k)}(\bar{\nu}) \geq 1$. By A.13), $P_{\ell-1}^{(k)}(\nu) \geq 2$, so by (A.11), $P_{\ell-1}^{(k)}(\nu)=$ $2, P_{\ell+1}^{(k)}(\nu)=0$ and $m_{\ell}\left(\nu^{(k+1)}\right)=0$ as above.

Next consider $m_{\ell}\left(\nu^{(k-1)}\right)=1$. Suppose that $P_{\ell-1}^{(k)}(\nu)=0$. By 3.9$)$

$$
P_{\ell-1}^{(k)}(\nu)=P_{\ell-1}^{(k)}(\bar{\nu})+\chi\left(\bar{\ell}^{(k-1)} \leq \ell-1\right) .
$$

Therefore $\bar{\ell}^{(k-1)} \geq \ell=\bar{\ell}^{(k)} \geq \bar{\ell}^{(k-1)}$, so that $\bar{\ell}^{(k-1)}=\ell$. Similarly $\widetilde{\ell}^{(k-1)}=\ell$. Then in $\nu^{(k-1)}$ there is only one string of length $\ell$ and both $\bar{\delta}$ and $\widetilde{\delta}$ select it. So for $\nu^{(k-1)}$ we are in Case 1 or Case 3. Suppose it is Case 3. Then $\widetilde{\bar{\ell}}^{(k-1)}=\overline{\tilde{\ell}}^{(k-1)}>\widetilde{\ell}^{(k-1)}=\bar{\ell}^{(k-1)}=\ell$ and $\widetilde{\ell}^{(k)} \geq \widetilde{\bar{\ell}}^{(k-1)}>\ell$ which is a contradiction. Suppose it is Case 1. Then $\widetilde{\bar{\ell}}^{(k-1)}=\widetilde{\ell}^{(k-1)}-1=\ell-1$. By (A.14), $P_{\ell-1}^{(k)}(\bar{\nu})=0$. Since $\bar{\ell}^{(k)}=\ell, m_{\ell-1}\left(\bar{\nu}^{(k)}\right) \geq 1$. So there is a singular string of length $\ell-1$ in $\bar{\nu}^{(k)}$ and $\widetilde{\bar{\ell}}^{(k-1)}=\ell-1$. By definition, $\widetilde{\bar{\ell}}^{(k)}=\ell-1$, which contradicts the assumption $\widetilde{\bar{\ell}}^{(k)}>\ell$.

Therefore $P_{\ell-1}^{(k)}(\nu) \geq 1$. By (A.11) it follows that $P_{\ell-1}^{(k)}(\nu)=1, P_{\ell+1}^{(k)}(\nu)=0$ and $m_{\ell}\left(\nu^{(k+1)}\right)=0$.

Finally consider $m_{\ell}\left(\nu^{(k-1)}\right)=2$. By A.11), $P_{\ell-1}^{(k)}(\nu)=0, P_{\ell+1}^{(k)}(\nu)=0$, and $m_{\ell}\left(\nu^{(k+1)}\right)=0$.

So $($ A.8 $),($ A.9 $)$, and $($ A.10 $)$ are proven.

Since $P_{\ell}^{(k)}(\nu)=P_{\ell+1}^{(k)}(\nu)=0$ (see (A.8)) it follows from Lemma 3.11, that if $p>\ell$ and $m_{n}\left(\nu^{(k)}\right)=0$ for all $\ell<n<p$, then $P_{n}^{(k)}(\nu)=0$ for $\ell \leq n \leq p$. Equation (3.10) furthermore implies that $m_{n}\left(\nu^{(k+1)}\right)=0$ for $\ell<n<p$.

Suppose $\nu^{(k)}$ has a string longer than $\ell$. Let $p$ be minimal such that $p>\ell$ and $m_{p}\left(\nu^{(k)}\right) \geq 1$. Since the string in $\nu^{(k)}$ of length $p$ is selected by neither $\bar{\delta}$ nor $\widetilde{\delta}$ but has vacancy number zero, its length remains $p$, its label stays zero in $\bar{\nu}^{(k)}$, and it remains singular in $\widetilde{\nu}^{(k)}$. Neither $\bar{\nu}^{(k)}$ nor $\widetilde{\nu}^{(k)}$ have strings of length $n$ for $\ell \leq n<p$. Since $\widetilde{\bar{\ell}}^{(k-1)} \leq \ell$ and $\overline{\tilde{\ell}}^{(k-1)} \leq \ell$ by (A.1) and since by assumption $\widetilde{\bar{\ell}}^{(k)}>\ell$ and $\overline{\tilde{\ell}}^{(k)}>\ell$, it follows that $\widetilde{\bar{\ell}}^{(k)}=p=\overline{\tilde{\ell}}^{(k)}$. Moreover, by the previous paragraph and (A.10), $m_{n}\left(\nu^{(k+1)}\right)=0$ for $\widetilde{\ell}^{(k)}=$ $\bar{\ell}^{(k)}=\ell \leq n<p$, so that $\tilde{\ell}^{(k+1)} \geq p$ and $\bar{\ell}^{(k+1)} \geq p$.

Otherwise there is no string in $\nu^{(k)}$ longer than $\ell$. Then $\widetilde{\bar{\ell}}^{(k)}=\overline{\bar{\ell}}^{(k)}=\infty$ and $\overline{\operatorname{rk}}(\widetilde{\nu}, \widetilde{J})=\widetilde{\operatorname{rk}}(\bar{\nu}, \bar{J})=k$. Moreover, the above result holds for all $p>\ell$, so that $m_{n}\left(\nu^{(k+1)}\right)=0$ for $n>\ell$. But by (A.10) $m_{\ell}\left(\nu^{(k+1)}\right)=0$ so that $\widetilde{\ell}^{(k+1)}=\bar{\ell}^{(k+1)}=\infty$ and $\overline{\operatorname{rk}}(\nu, J)=\widetilde{\operatorname{rk}}(\nu, J)=k+1$. 
By the appendix in [13] it follows that

$$
\lambda_{k}^{t}-\lambda_{k+1}^{t}=\lim _{n \rightarrow \infty} P_{n}^{(k)}(\nu) .
$$

But the right-hand side is zero so that $\lambda_{k}^{t}=\lambda_{k+1}^{t}$.

Proof of Lemma A.马. $\widetilde{\bar{\nu}}=\overline{\widetilde{\nu}}$ by Lemma A.2, whose entire proof will be used repeatedly without additional mention.

Selected strings. Consider a string in $(\nu, J)^{(k)}$ that is either selected by $\bar{\delta}$ or $\widetilde{\delta}$, or is such that its image under $\bar{\delta}$ (resp. $\widetilde{\delta}$ ) is selected by $\widetilde{\delta}$ (resp. $\bar{\delta}$ ). It is shown that the image of any such string under both $\widetilde{\delta} \circ \bar{\delta}$ and $\bar{\delta} \circ \widetilde{\delta}$, has the same label.

Selected strings, generic case. In the generic case for $\nu^{(k)}$, Remark A.1 shows that the string $\left(\widetilde{\ell}^{(k)}, 0\right) \in(\nu, J)^{(k)}$ is sent to the string $\left(\widetilde{\ell}^{(k)}-1,0\right)$ under either $\bar{\delta} \circ \widetilde{\delta}$ or $\widetilde{\delta} \circ \bar{\delta}$, and that a singular string of length $\bar{\ell}^{(k)}$ in $(\nu, J)^{(k)}$ is sent to a singular string of length $\bar{\ell}^{(k)}-1$ under either $\bar{\delta} \circ \widetilde{\delta}$ or $\widetilde{\delta} \circ \bar{\delta}$.

Selected strings, doubly singular case. Write $\ell=\widetilde{\ell}^{(k)}=\bar{\ell}^{(k)}$. The string $(\ell, 0) \in(\nu, J)^{(k)}$ is also singular.

Selected strings, Case 1. Here only the string $(\ell, 0) \in(\nu, J)^{(k)}$ and its images under $\bar{\delta}$ and $\widetilde{\delta}$ are selected. Moreover $P_{\ell-1}^{(k)}(\bar{\nu})=P_{\ell-1}^{(k)}(\widetilde{\nu})=0$ and $\widetilde{\bar{\ell}}^{(k)}=\overline{\bar{\ell}}^{(k)}=\ell-1$. The string $(\ell, 0)$ is sent to a string of length $\ell-2$ and singular label under $\bar{\delta} \circ \widetilde{\delta}$ and zero label under $\widetilde{\delta} \circ \bar{\delta}$. Hence it must be shown that $P_{\ell-2}^{(k)}(\overline{\widetilde{\nu}})=0$. Applying (3.9) and (3.16),

$$
\left.P_{\ell-2}^{(k)}(\overline{\widetilde{\nu}})=P_{\ell-2}^{(k)}(\nu)-\chi\left(\widetilde{\ell}^{(k-1)} \leq \ell-2\right)-\chi \overline{(\widetilde{\ell}}^{(k-1)} \leq \ell-2\right) .
$$

We divide into cases as in the proof of Case 1 in Lemma A.2. Suppose first that $P_{\ell-1}^{(k)}(\nu)=0$. Then $m_{\ell-1}\left(\nu^{(k)}\right)=0$, and applying (3.10) with $n=\ell-1$ one obtains $P_{\ell-2}^{(k)}(\nu)=0$. The admissibility of $\overline{\widetilde{\nu}}$ and (A.15) imply $P_{\ell-2}^{(k)}(\overline{\widetilde{\nu}})=0$.

By A.6) the only alternative is $P_{\ell-1}^{(k)}(\nu)=1$, which implies that $\widetilde{\ell}^{(k-1)} \leq$ $\ell-1$ and $\bar{\ell}^{(k-1)} \leq \ell-1$. By $\left(\right.$ A.7) $m_{\ell-1}\left(\nu^{(k-1)}\right) \leq 2$. As in the proof of Case 1 in Lemma A.2, we distinguish the three cases given by $m_{\ell-1}\left(\nu^{(k-1)}\right) \in$ $\{0,1,2\}$.

Suppose $m_{\ell-1}\left(\nu^{(k-1)}\right)=2$. By A.7 and (A.15) $P_{\ell-2}^{(k)}(\nu)=0$ and $P_{\ell-2}^{(k)}(\overline{\widetilde{\nu}})=0$.

Suppose $m_{\ell-1}\left(\nu^{(k-1)}\right)=1$. By $\left(\right.$ A.7) $P_{\ell-2}^{(k)}(\nu) \leq 1$. By (A.15) it suffices to show that either $\widetilde{\ell}^{(k-1)} \leq \ell-2$ or $\overline{\tilde{\ell}}^{(k-1)} \leq \ell-2$. Suppose neither holds. Then $\widetilde{\ell}^{(k-1)}>\ell-2$ and $\overline{\tilde{\ell}}^{(k-1)}>\ell-2$. Thus $\widetilde{\ell}^{(k-1)}=\ell-1$. Since 
$m_{\ell-1}\left(\nu^{(k-1)}\right)=1$ and $\widetilde{\ell}^{(k-1)}=\ell-1$, it follows that $m_{\ell-1}\left(\widetilde{\nu}^{(k-1)}\right)=0$. But then $\ell-1=\overline{\tilde{\ell}}^{(k)} \geq \overline{\tilde{\ell}}^{(k-1)}>\ell-2$, so $\overline{\bar{\ell}}^{(k-1)}=\ell-1$. However there are no strings of length $\ell-1$ in $\widetilde{\nu}^{(k-1)}$, which is a contradiction.

Suppose $m_{\ell-1}\left(\nu^{(k-1)}\right)=0$. By (A.7) $P_{\ell-2}^{(k)}(\nu) \leq 2$, so by A.15) it is enough to show that $\widetilde{\ell}^{(k-1)} \leq \ell-2$ and $\overline{\tilde{\ell}}^{(k-1)} \leq \ell-2$. In this subcase $\tilde{\ell}^{(k-1)} \leq \ell-2$ and $\bar{\ell}^{(k-1)} \leq \ell-2$. Suppose $\overline{\tilde{\ell}}^{(k-1)}>\ell-2$. Now $\ell-1=$ $\overline{\tilde{\ell}}^{(k)} \geq \overline{\bar{\ell}}^{(k-1)}>\ell-2$ so $\overline{\bar{\ell}}^{(k-1)}=\overline{\bar{\ell}}^{(k)}=\ell-1$. But $m_{\ell-1}\left(\nu^{(k-1)}\right)=0$ and $\tilde{\ell}^{(k-1)} \leq \ell-2$, so $m_{\ell-1}\left(\widetilde{\nu}^{(k-1)}\right)=0$, contradicting $\overline{\tilde{\ell}}^{(k-1)}=\ell-1$.

Selected strings, Case 2. Here there are two copies of the singular string $(\ell, 0) \in(\nu, J)^{(k)}$. If we think of $\bar{\delta}$ as selecting one of them and $\widetilde{\delta}$ the other, then the proof is the same as in the generic case.

Selected strings, Case 3. Let $p=\widetilde{\bar{\ell}}^{(k)}=\overline{\bar{\ell}}^{(k)}$. It satisfies $p>\ell$ and $P_{p}^{(k)}(\nu)=0$. Moreover $\widetilde{\ell}^{(k+1)} \geq p$ and $\bar{\ell}^{(k+1)} \geq p$. The strings that will be selected are singular strings $(\ell, 0)$ and $(p, 0)$ in $(\nu, J)^{(k)}$.

The string $(\ell, 0)$ maps to a string of length $\ell-1$, with label zero under $\bar{\delta} \circ \widetilde{\delta}$ and singular label under $\widetilde{\delta} \circ \bar{\delta}$. The string $(p, 0)$ is sent to a string of length $p-1$, with singular label under $\bar{\delta} \circ \widetilde{\delta}$ and to zero label under $\widetilde{\delta} \circ \bar{\delta}$.

It must be shown that

$$
\begin{aligned}
& P_{\ell-1}^{(k)}(\widetilde{\bar{\nu}})=0 \\
& P_{p-1}^{(k)}(\overline{\widetilde{\nu}})=0 .
\end{aligned}
$$

First (A.16) is established. By (3.9) and (3.16)

$$
P_{\ell-1}^{(k)}(\widetilde{\bar{\nu}})=P_{\ell-1}^{(k)}(\nu)-\chi\left(\widetilde{\bar{\ell}}^{(k-1)} \leq \ell-1\right)-\chi\left(\bar{\ell}^{(k-1)} \leq \ell-1\right) .
$$

By $\left(\right.$ A.9) $m_{\ell}\left(\nu^{(k-1)}\right) \leq 2$. We divide into cases for the choices of $m_{\ell}\left(\nu^{(k-1)}\right) \in$ $\{0,1,2\}$.

Suppose $m_{\ell}\left(\nu^{(k-1)}\right)=2$. By $\left(\right.$ A.9) $P_{\ell-1}^{(k)}(\nu)=0$. It follows immediately from the admissibility of $\widetilde{\bar{\nu}}$ and $\left(\right.$ A.18) that $P_{\ell-1}^{(k)}(\widetilde{\bar{\nu}})=0$.

Suppose $m_{\ell}\left(\nu^{(k-1)}\right)=1$. By $\left(\right.$ A.9) $P_{\ell-1}^{(k)}(\nu)=1$, so by (A.18) it is enough to show that either $\bar{\ell}^{(k-1)} \leq \ell-1$ or $\widetilde{\bar{\ell}}^{(k-1)} \leq \ell-1$. Suppose neither holds. Then $\ell=\bar{\ell}^{(k)} \geq \bar{\ell}^{(k-1)}>\ell-1$ so $\bar{\ell}^{(k-1)}=\ell$. Also by (A.1) $\ell=\widetilde{\ell}^{(k)} \geq \widetilde{\bar{\ell}}^{(k-1)}>\ell-1$ so that $\widetilde{\bar{\ell}}^{(k-1)}=\ell$. Now $m_{\ell}\left(\nu^{(k-1)}\right)=1$ and $\bar{\ell}^{(k-1)}=\ell$ so $m_{\ell}\left(\bar{\nu}^{(k-1)}\right)=0$, contradicting $\widetilde{\bar{\ell}}^{(k-1)}=\ell$.

Suppose $m_{\ell}\left(\nu^{(k-1)}\right)=0$. By (A.9) $P_{\ell-1}^{(k)}(\nu)=2$, so by (A.18), to prove (A.16) it is enough to show that $\bar{\ell}^{(k-1)} \leq \ell-1$ and $\widetilde{\bar{\epsilon}}^{(k-1)} \leq \ell-1$. By (A.12) 
we have $\bar{\ell}^{(k-1)} \leq \ell-1$ and $\widetilde{\ell}^{(k-1)} \leq \ell-1$. Suppose $\widetilde{\bar{\ell}}^{(k-1)}>\ell-1$. Since $\widetilde{\bar{\ell}}^{(k-1)} \leq \widetilde{\ell}^{(k)}=\ell$ by $\left(\right.$ A.1), it follows that $\widetilde{\bar{\ell}}^{(k-1)}=\ell$. Now $m_{\ell}\left(\nu^{(k-1)}\right)=0$ and $\bar{\ell}^{(k-1)} \leq \ell-1$ so $m_{\ell}\left(\bar{\nu}^{(k-1)}\right)=0$. This contradicts $\widetilde{\bar{\ell}}^{(k-1)}=\ell$.

Now let us prove (A.17). Using $p=\widetilde{\bar{\ell}}^{(k)}>\widetilde{\ell}^{(k)}$ and $\widetilde{\ell}^{(k+1)} \geq \widetilde{\bar{\ell}}^{(k)}=p$, by (3.9) and (3.16) we have

$$
P_{p-1}^{(k)}(\overline{\widetilde{\nu}})=P_{p-1}^{(k)}(\nu)-\chi\left(\overline{\tilde{\ell}}^{(k-1)} \leq p-1\right)+1
$$

Since $P_{p-1}^{(k)}(\nu)=0$, it must be shown that $\overline{\bar{\ell}}^{(k-1)} \leq p-1$. This is certainly the case, for otherwise by (A.1), $\ell=\bar{\ell}^{(k)} \geq \overline{\bar{\ell}}^{(k-1)} \geq p$, which is a contradiction.

Unselected strings. For the rest of the proof, assume that $(n, x)$ is a string in $(\nu, J)^{(k)}$ that is not selected by $\widetilde{\delta}$ or $\bar{\delta}$, and is such that its image under $\bar{\delta}$ (resp. $\widetilde{\delta}$ ) is not selected by $\widetilde{\delta}$ (resp. $\bar{\delta}$ ).

Using the fact that $\bar{\delta}$ preserves labels and $\widetilde{\delta}$ preserves colabels, it is enough to show that $P_{n}^{(k)}(\nu)-P_{n}^{(k)}(\widetilde{\nu})=P_{n}^{(k)}(\bar{\nu})-P_{n}^{(k)}(\widetilde{\bar{\nu}})$, which by (3.16) is equivalent to

$$
\begin{aligned}
& \chi\left(\widetilde{\ell}^{(k-1)} \leq n<\widetilde{\ell}^{(k)}\right)-\chi\left(\widetilde{\ell}^{(k)} \leq n<\widetilde{\ell}^{(k+1)}\right) \\
=\quad & \chi\left(\widetilde{\bar{\ell}}^{(k-1)} \leq n<\widetilde{\bar{\ell}}^{(k)}\right)-\chi\left(\widetilde{\bar{\ell}}^{(k)} \leq n<\widetilde{\bar{\ell}}^{(k+1)}\right) .
\end{aligned}
$$

Observe that for $a \leq b, \chi(a \leq n<b)=\chi(a \leq n)-\chi(b \leq n)$. Consider the functions

$$
\begin{aligned}
& \left.\Delta_{n}^{(k)}=\chi\left(\widetilde{\ell}^{(k)} \leq n\right)-\chi \widetilde{\bar{\ell}}^{(k)} \leq n\right) \\
& b_{n}^{-(k)}=\chi\left(m_{n}\left(\nu^{(k+1)}\right) \geq 1\right) \Delta_{n}^{(k)} \\
& b_{n}^{=(k)}=\chi\left(m_{n}\left(\nu^{(k)}\right) \geq 1\right) \Delta_{n}^{(k)} \\
& b_{n}^{+(k)}=\chi\left(m_{n}\left(\nu^{(k-1)}\right) \geq 1\right) \Delta_{n}^{(k)} .
\end{aligned}
$$

For parts $n$ that occur in $\nu^{(k)}$, A.19) is equivalent to

$$
b_{n}^{-(k-1)}-b_{n}^{=(k)}=b_{n}^{=(k)}-b_{n}^{+} \underset{n}{(k+1)} .
$$

It will be shown that $b_{n}^{-(k)}=b_{n}^{=(k)}=b_{n}^{+(k)}=0$ if $n$ is an unselected string in $\nu^{(k+1)}, \nu^{(k)}, \nu^{(k-1)}$, respectively, which implies (A.20).

Unselected strings, generic or Case 2. In this case $\widetilde{\bar{\ell}}^{(k)}=\widetilde{\ell}^{(k)}$, so that $\Delta_{n}^{(k)}=0$, and $b_{n}^{-(k)}=b_{n}^{=(k)}=b_{n}^{+(k)}=0$.

For the rest of the proof write $\ell=\widetilde{\ell}^{(k)}=\bar{\ell}^{(k)}$. 
Unselected strings, Case 1. Here $\widetilde{\widetilde{\ell}}^{(k)}=\ell-1$, so that $\Delta_{n}^{(k)}=-\chi(n=$ $\ell-1)$. Moreover $m_{\ell-1}\left(\nu^{(k)}\right)=0$ and $m_{\ell-1}\left(\nu^{(k+1)}\right)=0$. We have

$$
\begin{aligned}
& b_{n}^{=(k)}=-\chi(n=\ell-1) \chi\left(m_{\ell-1}\left(\nu^{(k)}\right) \geq 1\right)=0 \\
& b_{n}^{-(k)}=-\chi(n=\ell-1) \chi\left(m_{\ell-1}\left(\nu^{(k+1)}\right) \geq 1\right)=0 \\
& b_{n}^{+(k)}=-\chi(n=\ell-1) \chi\left(m_{\ell-1}\left(\nu^{(k-1)}\right) \geq 1\right) .
\end{aligned}
$$

It must be shown that $b_{n}^{+(k)}=0$ if $n$ is an unselected string in $\nu^{(k-1)}$ or equivalently that $m_{\ell-1}\left(\nu^{(k-1)}\right)=0$ if $\ell-1$ is an unselected string in $\nu^{(k-1)}$. Again we follow the division into cases of the proof of Lemma A.2.

0 .

If $P_{\ell-1}^{(k)}(\nu)=0$, it also follows by (3.10) with $n=\ell-1$ that $m_{\ell-1}\left(\nu^{(k-1)}\right)=$

Otherwise $P_{\ell-1}^{(k)}(\nu)=1$. We have $m_{\ell-1}\left(\nu^{(k)}\right)=0, \tilde{\ell}^{(k-1)} \leq \ell-1$ and $\bar{\ell}^{(k-1)} \leq \ell-1$. Also by $($ A.7)

$$
P_{\ell-2}^{(k)}(\nu) \leq 2-m_{\ell-1}\left(\nu^{(k-1)}\right) .
$$

Consider the three cases $m_{\ell-1}\left(\nu^{(k-1)}\right) \in\{0,1,2\}$.

If $m_{\ell-1}\left(\nu^{(k-1)}\right)=0$ there is nothing to show.

Suppose $m_{\ell-1}\left(\nu^{(k-1)}\right)=1$. It must be shown that there are no unselected strings of length $\ell-1$ in $\nu^{(k-1)}$. Thus it suffices to show that either $\widetilde{\ell}^{(k-1)}=$ $\ell-1$ or $\bar{\ell}^{(k-1)}=\ell-1$, for then the unique string in $\nu^{(k-1)}$ of length $\ell-1$ is selected. So assume that $\tilde{\ell}^{(k-1)} \leq \ell-2$ and $\bar{\ell}^{(k-1)} \leq \ell-2$. By A.21 $P_{\ell-2}^{(k)}(\nu) \leq 1$. Let $p$ be maximal such that $p<\ell-1$ and $m_{p}\left(\nu^{(k)}\right) \geq 1$; if it does not exist set $p=0$. If $p=\ell-2$ it follows from $P_{\ell-2}^{(k)}(\nu) \leq 1$, $\tilde{\ell}^{(k-1)} \leq \ell-2$ and $\bar{\ell}^{(k-1)} \leq \ell-2$ that either $\widetilde{\delta}$ or $\bar{\delta}$ selects a string of length $\ell-2$ in $\nu^{(k)}$ which contradicts the minimality of $\widetilde{\ell}^{(k)}$ and $\bar{\ell}^{(k)}$. Hence assume that $p<\ell-2$. By Lemma 3.11, $P_{n}^{(k)}(\nu)=1$ for $p+1 \leq n \leq \ell-1$ and $P_{p}^{(k)}(\nu) \leq 1$. Moreover by (3.10) $m_{n}\left(\nu^{(k-1)}\right)=m_{n}\left(\nu^{(k+1)}\right)=0$ for $p+2 \leq n \leq \ell-2$ and

$$
P_{p}^{(k)}(\nu)+m_{p+1}\left(\nu^{(k-1)}\right)+m_{p+1}\left(\nu^{(k+1)}\right) \leq 1 .
$$

Suppose $P_{p}^{(k)}(\nu)=1$. This case can only occur if $p \geq 1$. By (A.22) $m_{p+1}\left(\nu^{(k-1)}\right)=0$, so that $\widetilde{\ell}^{(k-1)} \leq p$ and $\bar{\ell}^{(k-1)} \leq p$. But $m_{p}\left(\nu^{(k)}\right) \geq 1$, so there is either a string in $\nu^{(k)}$ of length $p<\ell$ that is singular or of label 0 , contradicting the minimality of $\widetilde{\ell}^{(k)}$ and $\bar{\ell}^{(k)}$.

Therefore $P_{p}^{(k)}(\nu)=0$. If $\bar{\ell}^{(k-1)} \leq p$ (which can only happen if $p \geq 1$ ), since $m_{p}\left(\nu^{(k)}\right) \geq 1$ there is a singular string $(p, 0)$ in $(\nu, J)^{(k)}$, contradicting the definition of $\bar{\ell}^{(k)}=\ell$. So $\bar{\ell}^{(k-1)}>p$ and similarly $\tilde{\ell}^{(k-1)}>p$. Since it is assumed that $\bar{\ell}^{(k-1)} \leq \ell-2$ and $\widetilde{\ell}^{(k-1)} \leq \ell-2$, and there are no strings 
in $(\nu, J)^{(k-1)}$ of lengths $n$ such that $p+2 \leq n \leq \ell-2$, it follows that $\bar{\ell}^{(k-1)}=\widetilde{\ell}^{(k-1)}=p+1$ and $m_{p+1}\left(\nu^{(k-1)}\right)=1$.

This rules out the generic case and Case 2 for $\nu^{(k-1)}$. Case 1 for $\nu^{(k-1)}$ is also ruled out, for otherwise if $p \geq 1$ then by induction $m_{p}\left(\nu^{(k)}\right)=0$ which is a contradiction, and if $p=0$ then $\widetilde{\bar{\ell}}^{(k-1)}=\bar{\ell}^{(k-1)}-1=p=0$ which is also a contradiction.

Suppose Case 3 holds for $\nu^{(k-1)}$, that is, $\overline{\bar{\ell}}^{(k-1)}=\widetilde{\widetilde{\ell}}^{(k-1)}>\widetilde{\ell}^{(k-1)}=$ $\bar{\ell}^{(k-1)}=p+1$. But $\bar{\ell}^{(k-1)}=p+1$ and $m_{n}\left(\nu^{(k-1)}\right)=0$ for $p+2 \leq n \leq \ell-2$, so $m_{n}\left(\bar{\nu}^{(k-1)}\right)=0$ for $p+2 \leq n \leq \ell-2$. This implies $\widetilde{\bar{\ell}}^{(k-1)} \geq \ell-1$. Also $\ell-1=\widetilde{\bar{\ell}}^{(k)} \geq \widetilde{\bar{\ell}}^{(k-1)}$ so $\widetilde{\bar{\ell}}^{(k-1)}=\ell-1$. Thus the unique string in $(\nu, J)^{(k-1)}$ of length $\ell-1$ is selected while passing from $\bar{\delta}(\nu, J)$ to $\widetilde{\delta}(\bar{\delta}(\nu, J))$.

Suppose $m_{\ell-1}\left(\nu^{(k-1)}\right)=2$. By (A.21), $P_{\ell-2}^{(k)}(\nu)=0$. If $m_{\ell-2}\left(\nu^{(k)}\right)=0$ then by 3.10$)$ it follows that $P_{\ell-1}^{(k)}(\nu)=0$, which is a contradiction. Thus $m_{\ell-2}\left(\nu^{(k)}\right) \geq 1$. By the minimality of $\bar{\ell}^{(k)}=\widetilde{\ell}^{(k)}=\ell$, it follows that $\widetilde{\ell}^{(k-1)} \geq$ $\ell-1$ and $\bar{\ell}^{(k-1)} \geq \ell-1$. Since it is assumed that $\widetilde{\ell}^{(k-1)} \leq \ell-1$ and $\bar{\ell}^{(k-1)} \leq \ell-1$, we have $\bar{\ell}^{(k-1)}=\widetilde{\ell}^{(k-1)}=\ell-1$. It must be shown that both of the strings of length $\ell-1$ in $(\nu, J)^{(k-1)}$ get selected. Since $m_{\ell-1}\left(\nu^{(k-1)}\right)=2$, Case 3 does not hold for $\nu^{(k-1)}$. Case 1 does not hold either, for otherwise $m_{\ell-2}\left(\nu^{(k)}\right)=0$, which is a contradiction. Therefore either the generic case or Case 2 holds for $\nu^{(k-1)}$ and either way, both strings in $(\nu, J)^{(k-1)}$ of length $\ell-1$ are selected.

Unselected strings, Case 3. Here $\Delta_{n}^{(k)}=\chi\left(\ell \leq n<\widetilde{\bar{\ell}}^{(k)}\right)$. It follows from the proof of Lemma A.2 that $m_{n}\left(\nu^{(j)}\right)=0$ for $k-1 \leq j \leq k+1$ and $\ell<n<\widetilde{\bar{\ell}}^{(k)}$. Hence $b_{n}^{=(k)}=\chi(n=\ell) \chi\left(m_{\ell}\left(\nu^{(k)}\right) \geq 1\right), b_{n}^{-(k)}=\chi(n=$ $\ell) \chi\left(m_{\ell}\left(\nu^{(k+1)}\right) \geq 1\right)$, and $b_{n}^{+(k)}=\chi(n=\ell) \chi\left(m_{\ell}\left(\nu^{(k-1)}\right) \geq 1\right)$. However in Case $3, m_{\ell}\left(\nu^{(k)}\right)=1$, so that there are no other strings in $(\nu, J)^{(k)}$ of length $\ell$ other than the one selected by $\bar{\delta}$ and $\widetilde{\delta}$. So $b_{n}^{=(k)}=0$ for all applicable strings $(n, J)$. By (A.10) also $b_{n}^{-(k)}=0$.

It is enough to show that $b_{n}^{+(k)}=0$, that is, $m_{\ell}\left(\nu^{(k-1)}\right)=0$ if $\ell$ is an unselected string. By (A.9) we distinguish the cases $m_{\ell}\left(\nu^{(k-1)}\right) \in\{0,1,2\}$. If $m_{\ell}\left(\nu^{(k-1)}\right)=0$ we are done.

Suppose that $m_{\ell}\left(\nu^{(k-1)}\right)=1$. By definition $\bar{\ell}^{(k-1)} \leq \ell$ and $\widetilde{\ell}^{(k-1)} \leq \ell$. If $\widetilde{\ell}^{(k-1)}=\ell$ or $\bar{\ell}^{(k-1)}=\ell$ then the only string of length $\ell$ in $(\nu, J)^{(k-1)}$ is selected. So suppose $\bar{\ell}^{(k-1)} \leq \ell-1$. Then by $\left(\right.$ A.9) and A.14 $P_{\ell-1}^{(k)}(\bar{\nu})=0$. Since $m_{\ell-1}\left(\bar{\nu}^{(k)}\right) \geq 1$ and $\widetilde{\bar{\ell}}^{(k)}>\ell$, it follows that $\widetilde{\bar{\ell}}^{(k-1)} \geq \ell$. On the other 
hand $\widetilde{\bar{\ell}}^{(k-1)} \leq \widetilde{\ell}^{(k)}=\ell$ by $\left(\right.$ A.1), so $\widetilde{\bar{\ell}}^{(k-1)}=\ell$. Thus the lone string in $\nu^{(k-1)}$ of length $\ell$ is selected in passing from $\bar{\nu}^{(k-1)}$ to $\widetilde{\bar{\nu}}^{(k-1)}$.

Finally suppose $m_{\ell}\left(\nu^{(k-1)}\right)=2$. By $\left(\right.$ A.9) $P_{\ell-1}^{(k)}(\nu)=0$. It follows from (A.14) that $P_{\ell-1}^{(k)}(\bar{\nu})=0$ and $\bar{\ell}^{(k-1)} \geq \ell$. Since $\bar{\ell}^{(k-1)} \leq \bar{\ell}^{(k)}=\ell$, we have $\bar{\ell}^{(k-1)}=\ell$. Similarly $\widetilde{\ell}^{(k-1)}=\ell$. It is enough to show that either the generic case or Case 2 holds for $\nu^{(k-1)}$ for then both strings of length $\ell$ in $\nu^{(k-1)}$ are

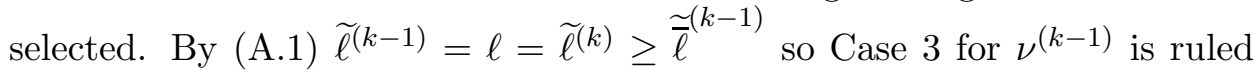
out. Suppose Case 1 holds for $\nu^{(k-1)}$. Then $\bar{\ell}^{(k-1)}=\widetilde{\ell}^{(k-1)}=\ell$ and $\widetilde{\bar{\ell}}^{(k-1)}=$ $\widetilde{\ell}^{(k-1)}-1=\ell-1$. By (3.9) with $n=\ell-1, P_{\ell-1}^{(k)}(\nu)=P_{\ell-1}^{(k)}(\bar{\nu})=0$. Now $m_{\ell-1}\left(\bar{\nu}^{(k)}\right) \geq 1$, so there is a string $(\ell-1,0) \in(\bar{\nu}, \bar{J})^{(k)}$. But $\ell-1={\widetilde{\widetilde{\ell}^{(k-1)}}}^{(k)}$ so this is a contradiction to the definition of $\widetilde{\bar{\ell}}^{(k)}>\ell$.

\section{Appendix B. Proof of Lemma 7.3}

Lemma 7.3 is established here by Corollary B.6 and Lemma B.7.

Let $R=\left(R_{1}, \ldots, R_{L}\right)$ such that $R_{j}=\left(\eta_{j}^{\mu_{j}}\right)$ with $\eta_{L}=1, \lambda$ a partition and $(\nu, J) \in \operatorname{RC}\left(\lambda^{t} ; R^{t}\right)$. Denote $(\bar{\nu}, \bar{J})=\bar{\delta}(\nu, J) \in \operatorname{RC}\left(\rho^{t} ; \bar{R}^{t}\right)$ and $\left(\nu^{t}, J^{t}\right)=$ $\operatorname{tr}_{\mathrm{RC}}(\nu, J) \in \mathrm{RC}(\lambda ; R)$. Let $\bar{\ell}^{(k)}$ be the lengths of the strings selected by $\bar{\delta}$ and $r=\overline{\operatorname{rk}}(\nu, J)$, the minimum index such that $\bar{\ell}^{(r)}=\infty$. By Proposition 3.12 the partition $\rho$ is obtained by removing the corner cell $\left(\lambda_{r}^{t}, r\right)$ of $\lambda$.

Recall that to every configuration $\nu$ there is an associated matrix (7.1). Let $\bar{\delta} m$ be the matrix of $\bar{\nu}$. Then for all $i, j \geq 1$,

$$
m_{i j}-(\bar{\delta} m)_{i j}=\chi(i-1 \geq 1) \chi\left(j=\bar{\ell}^{(i-1)}\right)-\chi\left(j=\bar{\ell}^{(i)}\right) .
$$

The matrix of the RC-transpose of $\bar{\delta} m$ can be calculated as follows.

$$
\begin{aligned}
\left((\bar{\delta} m)^{t}\right)_{i j} & =-(\bar{\delta} m)_{j i}+\chi((i, j) \in \rho)-\sum_{a=1}^{L} \chi\left((i, j) \in \bar{R}_{a}\right) \\
& =-m_{j i}+\chi(j-1 \geq 1) \chi\left(i=\bar{\ell}^{(j-1)}\right)-\chi\left(i=\bar{\ell}^{(j)}\right)+\chi((i, j) \in \lambda) \\
& -\chi\left((i, j)=\left(\lambda_{r}^{t}, r\right)\right)-\sum_{a=1}^{L} \chi\left((i, j) \in R_{a}\right)+\chi\left((i, j)=\left(\mu_{L}, 1\right)\right) \\
& =m_{i j}^{t}+\chi\left(i=\bar{\ell}^{(j-1)}\right)-\chi\left(i=\bar{\ell}^{(j)}\right)-\chi\left((i, j)=\left(\lambda_{r}^{t}, r\right)\right) .
\end{aligned}
$$

Let $\partial m^{t}$ be the matrix of the configuration of $\partial\left(\nu^{t}, J^{t}\right)$ and $\ell^{(k)}$ the lengths of the strings selected by $\partial$. Then

$$
\left(\partial m^{t}\right)_{i j}=m_{i j}^{t}-\chi\left(i-1 \geq \mu_{L}\right) \chi\left(j=\ell^{(i-1)}\right)+\chi\left(i \geq \mu_{L}\right) \chi\left(j=\ell^{(i)}\right) .
$$


Thus the configurations of $\partial \circ \operatorname{tr}_{\mathrm{RC}}(\nu, J)$ and $\operatorname{tr}_{\mathrm{RC}} \circ \bar{\delta}(\nu, J)$ coincide, if and only if

$$
\begin{aligned}
& -\chi\left(i-1 \geq \mu_{L}\right) \chi\left(j=\ell^{(i-1)}\right)+\chi\left(i \geq \mu_{L}\right) \chi\left(j=\ell^{(i)}\right) \\
& =\chi\left(i=\bar{\ell}^{(j-1)}\right)-\chi\left(i=\bar{\ell}^{(j)}\right)-\chi\left((i, j)=\left(\lambda_{r}^{t}, r\right)\right) .
\end{aligned}
$$

Remark B.1. By the definition of the RC-transpose map, there cannot be a singular string of length $n$ in $\nu^{(k)}$ and a singular string of length $k$ in $\nu^{t(n)}$.

Remark B.2. Let $i, j \geq 1$. Suppose $(i, j+1) \in \lambda$, that is, $\lambda_{j+1}^{t} \geq i$. Then by [13, (9.7)] for the admissible $\left(\lambda^{t} ; R^{t}\right)$-configuration $\nu$, we have $m_{j}\left(\nu^{t(i)}\right)=$ $P_{i}^{(j)}(\nu)$.

Similarly if $(i+1, j) \in \lambda$ then $m_{i}\left(\nu^{(j)}\right)=P_{j}^{(i)}\left(\nu^{t}\right)$.

Lemma B.3. Suppose that $\nu$ is an admissible configuration of type $\left(\lambda^{t} ; R^{t}\right)$. Then for all $n \geq \lambda_{k}^{t}, P_{n}^{(k)}(\nu)=\lambda_{k}^{t}-\lambda_{k+1}^{t}$.

Proof. By [13, (11.2)], we have

$$
P_{n}^{(k)}(\nu)=\lambda_{k}^{t}-\lambda_{k+1}^{t}+\sum_{a=1}^{L} \min \left(0, n-\mu_{a}\right) \delta_{\eta_{a}, k}
$$

whenever $n \geq \nu_{1}^{(k-1)}, n \geq \nu_{1}^{(k)}$, and $n \geq \nu_{1}^{(k+1)}$. But in the proof of 13 , Lemma 10] it is shown that $\lambda_{p+1}^{t} \geq \nu_{1}^{(p)}$ for all $p$, and in particular for $k-1 \leq p \leq k+1$. Thus for $n \geq \lambda_{k}^{t}$, (B.2) holds. It suffices to show that the sum over $a$ is zero. Suppose not. Then there is a rectangle $R_{a}$ in $R$ whose width is $\eta_{a}=k$ and its height $\mu_{a}$ satisfies $\mu_{a}>n \geq \lambda_{k}^{t}$. This means that $R_{a}$ is not contained in $\lambda$, which contradicts the assumption that $\operatorname{RC}\left(\lambda^{t} ; R^{t}\right)$ is nonempty.

Define the sets of pairs of positive integers

$$
\begin{aligned}
& H_{\delta}=\left\{(i, j) \mid i=\bar{\ell}^{(j)}\right\} \\
& H_{\partial}=\left\{(i, j) \mid j=\ell^{(i)}\right\} .
\end{aligned}
$$

Let $H=H_{\delta} \cup H_{\partial}$ and $H^{+}=H \cup\left\{\left(\lambda_{r}^{t}, r\right)\right\}$.

Remark B.4. $H \subset \lambda$. To see this, by [13, Lemma 10], $m_{n}\left(\nu^{(k)}\right)>0$ implies that $n \leq \lambda_{k+1}^{t}$, that is, $(n, k+1) \in \lambda$ and hence $(n, k) \in \lambda$. Likewise, $m_{k}\left(\nu^{t(n)}\right)>0$ implies $k \leq \lambda_{n+1}$, that is, $(n+1, k) \in \lambda$ and hence $(n, k) \in \lambda$. This also shows that every cell of $H_{\delta}$ is not at the end of its row in $\lambda$ and every cell of $H_{\partial}$ is not at the bottom of its column in $\lambda$.

Lemma B.5. $\left(\lambda_{r}^{t}, r\right) \notin H$ and $H^{+}$is a rookwise connected subset of the Ferrers diagram of $\lambda$ that can be viewed as leading from the cell $(1,1)$ to the cell $\left(\lambda_{r}^{t}, r\right)$ where cells of $H_{\delta}$ are viewed as commands to proceed to the east by one column and cells of $H_{\partial}$ are commands to proceed south by a row. 
Proof. Define the cell $s_{i}=(i, 1)$ for $1 \leq i \leq \mu_{L}-1 ; s_{i} \in H_{\partial}$ by definition. Also $s_{i} \in \lambda$ since $\operatorname{RC}\left(\lambda^{t} ; R^{t}\right)$ is assumed to be nonempty which by the Littlewood-Richardson rule implies that $\lambda$ contains $R_{L}=\left(1^{\mu_{L}}\right)$. By induction suppose that the cell $s_{m-1} \in H \cap \lambda$ has been defined for $m \geq \mu_{L}$. Let $s_{m}$ be the cell just east of $s_{m-1}$ if $s_{m-1} \in H_{\delta}$ and the cell just south of $s_{m-1}$ if $s_{m-1} \in H_{\partial}$. This is well-defined since $H_{\delta} \cap H_{\partial}=\emptyset$ by Remark B.1. Moreover $s_{m} \in \lambda$ by Remark B.4. If $\mu_{L}>1$ the algorithm defines $s_{\mu_{L}}=\left(\mu_{L}, 1\right)$. If $\mu_{L}=1$ we define $s_{1}=(1,1)$.

We shall show that one of three possibilities must occur, which are mutually disjoint by Remarks B.1 and B.4.

1. $s_{m}$ is a corner cell of $\lambda$.

2. $s_{m} \in H_{\delta}$.

3. $s_{m} \in H_{\partial}$.

Write $s_{m}=(i, j)$.

Suppose that there is a singular string in $(\nu, J)^{(j)}$ of length $i$. Then it will be shown that $i=\bar{\ell}^{(j)}$ and $(i, j) \in H_{\delta}$. By construction either $s_{m-1}=(i, j-1)$ or $s_{m-1}=(i-1, j)$. Suppose that $s_{m-1}=(i, j-1)$, that is, $i=\bar{\ell}^{(j-1)}$. Then by definition $i=\bar{\ell}^{(j)}$ and $(i, j) \in H_{\delta}$. Otherwise $s_{m-1}=(i-1, j)$. By definition $\left(\bar{\ell}^{(j-1)}, j-1\right) \in H_{\delta}$ and by the construction of $s_{1}$ through $s_{m}$ we have $\left(i^{\prime}, j\right) \in H_{\partial}$ for $\bar{\ell}^{(j-1)} \leq i^{\prime} \leq i-1$, for otherwise the path would have proceeded into column $j+1$. In particular for such $i^{\prime}$ there are no singular strings of length $i^{\prime}$ in $(\nu, J)^{(j)}$ by Remark B.1, so that by definition $i=\bar{\ell}^{(j)}$.

Similarly if there is a singular string in $\left(\nu^{t}, J^{t}\right)^{(i)}$ of length $j$ then $j=\ell^{(i)}$ and $(i, j) \in H_{\partial}$.

Otherwise it may be assumed that there is not a singular string in $(\nu, J)^{(j)}$ of length $i$ and there is not a singular string in $\left(\nu^{t}, J^{t}\right)^{(i)}$ of length $j$.

Under these assumptions, the following situations lead to contradictions:

$$
\begin{aligned}
& m_{i}\left(\nu^{(j)}\right)=P_{i}^{(j)}(\nu)=0 \\
& m_{j}\left(\nu^{t(i)}\right)=P_{j}^{(i)}\left(\nu^{t}\right)=0 .
\end{aligned}
$$

For suppose (B.3) holds. In the base case $m=\mu_{L}$, we have $(i, j)=\left(\mu_{L}, 1\right)$. Recall that $R_{L}=\left(1^{\mu_{L}}\right)$. This implies that $m_{\mu_{L}}\left(\xi^{(1)}(R)\right)>0$. In particular it follows immediately from (3.10) with $k=j=1$ and $n=i=\mu_{L}$ that (B.3) is impossible.

Otherwise assume that we are in the inductive case $m>\mu_{L}$. Applying (3.10) with $n=i$ and $k=j$, it follows that $m_{i}\left(\nu^{(j-1)}\right)=0$ and $P_{i-1}^{(j)}(\nu)=0$. The former immediately implies $(i, j-1) \notin H$. By construction, since $(i, j-1) \notin H$, it must be the case that $s_{m-1}=(i-1, j) \in H_{\partial}$. Thus there is a singular string of length $j$ in $\left(\nu^{t}, J^{t}\right)^{(i-1)}$, so that $m_{j}\left(\nu^{t(i-1)}\right)>0$. By $(\overline{7.3}), m_{i-1}\left(\nu^{(j)}\right)=P_{j}^{(i-1)}\left(\nu^{t}\right)$. Suppose this common value is nonzero. By $(7.3), P_{i-1}^{(j)}(\nu)=m_{j}\left(\nu^{t(i-1)}\right)>0$, which is a contradiction. Therefore 
$m_{i-1}\left(\nu^{(j)}\right)=0$. But also $P_{i-1}^{(j)}(\nu)=0$, so that $(\mathrm{B} .3)$ holds for $(i-1, j)$. But this is a contradiction by induction since $(i-1, j)$ is earlier in the path than $(i, j)$.

Similarly (B.4) leads to a contradiction.

Now the proof divides into four cases depending on whether the cells $(i+1, j)$ and $(i, j+1)$ are in $\lambda$ or not.

Suppose first that both $(i, j+1) \in \lambda$ and $(i+1, j) \in \lambda$. By Remark B.2 we have $a:=m_{i}\left(\nu^{(j)}\right)=P_{j}^{(i)}\left(\nu^{t}\right)$ and $b:=m_{j}\left(\nu^{t(i)}\right)=P_{i}^{(j)}(\nu)$. If $a>0$ and $b>0$ then by the definition of the RC-transpose map on riggings, there is either a singular string of length $i$ in $(\nu, J)^{(j)}$ or a singular string of length $j$ in $\left(\nu^{t}, J^{t}\right)^{(i)}$, contradicting a previous assumption. If $a>0$ and $b=0$ then there is a string of length $i$ in $(\nu, J)^{(j)}$ with vacancy number zero, so it is singular, which is a contradiction. A similar contradiction is reached if $a=0$ and $b>0$. If $a=b=0$ then one obtains the impossibility (B.3).

Next suppose $(i, j+1) \in \lambda$ and $(i+1, j) \notin \lambda$ so that $\lambda_{j}^{t}=i=\lambda_{j+1}^{t}$. By Lemma B.3 with $k=j$ and $n=i=\lambda_{j}^{t}$ we have $P_{i}^{(j)}(\nu)=0$. If $m_{i}\left(\nu^{(j)}\right)>0$ then there is a singular string of length $i$ in $(\nu, J)^{(j)}$ which is a contradiction, and if $m_{i}\left(\nu^{(j)}\right)=0$ one obtains the impossibility $(\mathrm{B} .3)$.

In a similar manner one rules out the case $(i, j+1) \notin \lambda$ and $(i+1, j) \in \lambda$.

The last remaining case is $(i, j+1) \notin \lambda$ and $(i+1, j) \notin \lambda$. But $(i, j) \in \lambda$ so $(i, j)$ is a corner cell of $\lambda$. This finishes the proof of the trichotomy.

Now it is enough to show that if $s_{m}=(i, j)$ is a corner cell of $\lambda$ then $j=r=\overline{\operatorname{rk}}(\nu, J)$. There cannot be a cell of $H_{\delta}$ in the $j$-th column or the path would have crossed into the $(j+1)$-st column. Thus $j \geq r$. If $j>1$ then by construction there is a cell of $H_{\delta}$ in the $(j-1)$-st column, so $j \leq r$ and therefore $j=r$. If $j=1$ then $H_{\delta}$ is empty and $\bar{\ell}^{(1)}=\infty$, and again $j=r$ since both equal 1 .

Corollary B.6. The configurations of $\operatorname{tr}_{\mathrm{RC}} \circ \bar{\delta}(\nu, J)$ and $\partial \circ \operatorname{tr}_{\mathrm{RC}}(\nu, J)$ coincide.

Proof. Follows directly from (B.1) and Lemma B.5.

Lemma B.7. The riggings of $\operatorname{tr}_{\mathrm{RC}} \circ \bar{\delta}(\nu, J)$ and $\partial \circ \operatorname{tr}_{\mathrm{RC}}(\nu, J)$ coincide.

Proof. Let $(\bar{\nu}, \overline{\mathrm{J}})=\bar{\delta}(\nu, J),\left(\nu^{t}, J^{t}\right)=\operatorname{tr}_{\mathrm{RC}}(\nu, J),\left(\bar{\nu}^{t}, \overline{\mathrm{J}}^{t}\right)=\operatorname{tr}_{\mathrm{RC}} \circ \bar{\delta}(\nu, J)$ and $\left(\overline{\nu^{t}}, \overline{J^{t}}\right)=\partial \circ \operatorname{tr}_{\mathrm{RC}}(\nu, J)$. Denote the corresponding partitions by $\overline{\mathrm{J}}_{n}^{(k)}$, $J_{n}^{t(k)}, \overline{\mathrm{J}}_{n}^{t(k)}$ and ${\overline{J^{t}}}_{n}^{(k)}$, respectively. The aim is to show that $\overline{\mathrm{J}}_{k}^{t(n)}={\overline{J^{t}}}_{k}^{(n)}$ for all $n, k \geq 1$. Since the transpose of a rigging depends on the vacancy number and the vacancy number changes under $\bar{\delta}$ according to (3.9) we need to distinguish several ranges for $n$.

First suppose that $\bar{\ell}^{(k-1)} \leq n<\bar{\ell}^{(k)}-1$. In this case the riggings are not changed by $\bar{\delta}$ so that $J_{n}^{(k)}=\overline{\mathrm{J}}_{n}^{(k)}$ and furthermore the largest part of $J_{n}^{(k)}$ is smaller than $P_{n}^{(k)}(\nu)$ since by the definition of $\bar{\ell}^{(k)}$ there are no singular 
strings in the range of $n$. The rectangle corresponding to $\overline{\mathrm{J}}_{n}^{(k)}$ has height $m_{n}\left(\bar{\nu}^{(k)}\right)=m_{n}\left(\nu^{(k)}\right)$ and width $P_{n}^{(k)}(\bar{\nu})=P_{n}^{(k)}(\nu)-1$ by (3.9). Hence, compared to $J_{k}^{t(n)}, \bar{J}_{k}^{t(n)}$ has one part of length $m_{n}\left(\nu^{(k)}\right)$ less. It follows from Lemma B.5 that $\ell^{(n)}=k$. Since furthermore $\mu_{L} \leq \bar{\ell}^{(k-1)} \leq n$, this implies that $\partial$ removes one part of length $P_{k}^{(n)}\left(\nu^{t}\right)$ from $J_{k}^{t(n)}$. It also ensures that $m_{k}\left(\nu^{t(n)}\right)>0$. By $(\overline{7.3}), P_{k}^{(n)}\left(\nu^{t}\right)=m_{n}\left(\nu^{(k)}\right)$ which proves $\bar{J}_{k}^{t(n)}={\overline{J^{t}}}_{k}^{(n)}$.

Next suppose that $\bar{\ell}^{(k)}<n<\bar{\ell}^{(k+1)}$. Again by the choice of $n$ we have $J_{n}^{(k)}=\bar{J}_{n}^{(k)}$, and the rectangle containing $\bar{J}_{n}^{(k)}$ has height $m_{n}\left(\bar{\nu}^{(k)}\right)=m_{n}\left(\nu^{(k)}\right)$ and width $P_{n}^{(k)}(\bar{\nu})=P_{n}^{(k)}(\nu)+1$ by $(3.9)$. Hence, compared to $J_{k}^{t(n)}, \overline{\mathrm{J}}_{k}^{t(n)}$ has an extra part of size $m_{n}\left(\nu^{(k)}\right)$. By Lemma B.5, $k=\ell^{(n)}-1$ so that $\partial$ adds an extra part of size $P_{k}^{(n)}\left(\overline{\nu^{t}}\right)$ to $J_{k}^{t(n)}$ and $m_{k}\left({\overline{\nu^{t}}}^{(n)}\right)>0$. By Corollary B.6 this also implies that $m_{k}\left(\bar{\nu}^{t(n)}\right)>0$. Hence one finds by $(7.3)$ that $P_{k}^{(n)}\left(\overline{\nu^{t}}\right)=P_{k}^{(n)}\left(\bar{\nu}^{t}\right)=m_{n}\left(\bar{\nu}^{(k)}\right)=m_{n}\left(\nu^{(k)}\right)$. This shows that $\overline{\mathrm{J}}_{k}^{t(n)}={\overline{J^{t}}}_{k}^{(n)}$.

Now assume that $n=\bar{\ell}^{(k)}$ and $\bar{\ell}^{(k)}<\bar{\ell}^{(k+1)}$. Since $n=\bar{\ell}^{(k)}$ the largest part of $J_{n}^{(k)}$ equals $P_{n}^{(k)}(\nu)$ which is removed under $\bar{\delta}$. The rectangle corresponding to $\bar{J}_{n}^{(k)}$ has height $m_{n}\left(\bar{\nu}^{(k)}\right)=m_{n}\left(\nu^{(k)}\right)-1$ and width $P_{n}^{(k)}(\bar{\nu})=$ $P_{n}^{(k)}(\nu)+1$. Hence, compared to $J_{k}^{t(n)}, \bar{J}_{k}^{t(n)}$ has an extra part of size $m_{n}\left(\nu^{(k)}\right)-1$. From Lemma B.5 we find that $\ell^{(n)}=k+1$ so that $\partial$ adds a part of size $P_{k}^{(n)}\left(\overline{\nu^{t}}\right)$ to $J_{k}^{t(n)}$. Since $\partial$ added a part, $m_{k}\left(\bar{\nu}^{t(n)}\right)=m_{k}\left({\overline{\nu^{t}}}^{(n)}\right)>0$ which by (7.3) implies that $P_{k}^{(n)}\left(\overline{\nu^{t}}\right)=m_{n}\left(\nu^{(k)}\right)-1$. Hence $P_{k}^{(n)}\left(\overline{\nu^{t}}\right)=$ $P_{k}^{(n)}\left(\bar{\nu}^{t}\right)=m_{n}\left(\bar{\nu}^{(k)}\right)=m_{n}\left(\nu^{(k)}\right)-1$ by $(\overline{7.3})$.

Next assume that $n=\bar{\ell}^{(k)}=\bar{\ell}^{(k+1)}$. Again $\overline{\mathrm{J}}_{n}^{(k)}$ is obtained from $J_{n}^{(k)}$ by removing a part of size $P_{n}^{(k)}(\nu)$, and this time the rectangle corresponding to $\bar{J}_{n}^{(k)}$ has height $m_{n}\left(\bar{\nu}^{(k)}\right)=m_{n}\left(\nu^{(k)}\right)-1$ and width $P_{n}^{(k)}(\bar{\nu})=P_{n}^{(k)}(\nu)$. Therefore $\overline{\mathrm{J}}_{k}^{t(n)}=J_{k}^{t(n)}$. By Lemma B.5 we have $\ell^{(n-1)} \leq k<\ell^{(n)}-1$ so that also ${\overline{J^{t}}}_{k}^{(n)}=J_{k}^{t(n)}$ which proves the assertion.

Consider the case $n=\bar{\ell}^{(k)}-1$ and $\bar{\ell}^{(k-1)}<\bar{\ell}^{(k)}$. By the assumptions on $n$ there is no singular string of length $n$ in $\nu^{(k)}$ so that the largest part of $J_{n}^{(k)}$ is smaller than $P_{n}^{(k)}(\nu)$. Under $\bar{\delta}$ a part of size $P_{n}^{(k)}(\bar{\nu})=P_{n}^{(k)}(\nu)-1$ gets added to $J_{n}^{(k)}$, and the rectangle corresponding to $\bar{J}_{n}^{(k)}$ has height $m_{n}\left(\bar{\nu}^{(k)}\right)=$ $m_{n}\left(\nu^{(k)}\right)+1$ and width $P_{n}^{(k)}(\nu)-1$. Hence, compared to $J_{k}^{t(n)}$, a part of size $m_{n}\left(\nu^{(k)}\right)$ is missing in $\overline{\mathrm{J}}_{k}^{t(n)}$. By Lemma B.5 $\ell^{(n)}=k$. Hence $\partial$ removes a part of size $P_{k}^{(n)}\left(\nu^{t}\right)$ from $J_{k}^{t(n)}$. But $P_{k}^{(n)}\left(\nu^{t}\right)=m_{n}\left(\nu^{(k)}\right)$ by (7.3) because $m_{k}\left(\nu^{t(n)}\right)>0$ since $k=\ell^{(n)}$.

Now assume that $n+1=\bar{\ell}^{(k)}=\bar{\ell}^{(k-1)}$. The partition $\overline{\mathrm{J}}_{n}^{(k)}$ is obtained from $J_{n}^{(k)}$ by adding a part of size $P_{n}^{(k)}(\bar{\nu})=P_{n}^{(k)}(\nu)$ and its rectangle has height 
$m_{n}\left(\bar{\nu}^{(k)}\right)=m_{n}\left(\nu^{(k)}\right)+1$ and width $P_{n}^{(k)}(\nu)$. Hence $\overline{\mathrm{J}}_{k}^{t(n)}=J_{k}^{t(n)}$. By Lemma B.5, $\ell^{(n)}<k<\ell^{(n+1)}$ if $k>1$ which implies that $\partial$ preserves the rigging so that ${\overline{J^{t}}}_{k}^{(n)}=J_{k}^{t(n)}$ proving the assertion. If $k=1$ then $n=\bar{\ell}^{(0)}-1=\mu_{L}-1$. In this case $\partial$ also does not change the rigging so that ${\overline{J^{t}}}_{k}^{(n)}=J_{k}^{t(n)}$.

Finally assume that none of the above cases holds for $n$ so that $m_{n}\left(\bar{\nu}^{(k)}\right)=$ $m_{n}\left(\nu^{(k)}\right), P_{n}^{(k)}(\bar{\nu})=P_{n}^{(k)}(\nu)$ and $\overline{\mathbf{J}}_{n}^{(k)}=J_{n}^{(k)}$ which implies that $\overline{\mathrm{J}}_{k}^{t(n)}=J_{k}^{t(n)}$. If $n \geq \bar{\ell}^{(k+1)}$ one finds by Lemma B.5 that $k \leq \ell^{(n)}-2$. If $n<\bar{\ell}^{(k-1)}$ for $k>1$, Lemma B.5 implies that $k>\ell^{(n)}$. The remaining case is $n<\bar{\ell}^{(0)}=$ $\mu_{L}$. In all three cases $\partial$ does not alter $J_{k}^{t(n)}$ so that indeed ${\overline{J^{t}}}_{k}^{(n)}=\overline{\mathrm{J}}_{k}^{t(n)}$.

\section{REFERENCES}

[1] A. Berkovich, Fermionic counting of RSOS-states and Virasoro character formulas for the unitary minimal series $M(\nu, \nu+1)$. Exact results, Nucl. Phys. B 431 (1994) 315-348.

[2] P. Bouwknegt, A. W. W. Ludwig and K. Schoutens, Spinon bases, Yangian symmetry and fermionic representations of Virasoro characters in conformal field theory, Phys. Lett. B338 (1994) 448-456.

[3] A. Berkovich, B. M. McCoy and A. Schilling, Rogers-Schur-Ramanujan type identities for the $M\left(p, p^{\prime}\right)$ minimal models of conformal field theory, Commun. Math. Phys. 191 (1998) 325-395.

[4] S. Dasmahapatra and O. Foda, Strings, paths and standard tableaux, Int. J. Mod. Phys. A13 (1998) 501-522.

[5] O. Foda, K. S. M. Lee and T. A. Welsh, A Burge tree of Virasoro-type polynomial identities, preprint $\mathrm{a-alg/9710025.}$

[6] S. Fomin and C. Greene, A Littlewood-Richardson miscellany, European J. Combin. 14 (1993) 191-212.

[7] G. Hatayama, A. N. Kirillov, A. Kuniba, M. Okado, T. Takagi and Y. Yamada, Character formulae of $\widehat{s l}_{n}$-modules and inhomogeneous paths, Nucl. Phys. B536 [PM] (1998) 575-616.

[8] G. Hatayama, A. Kuniba, M. Okado, T. Takagi and Y. Yamada, Remarks on fermionic formula, preprint math.QA/9812022.

[9] R. Kedem, T. R. Klassen, B. M. McCoy and E. Melzer, Fermionic quasi-particle representations for characters of $\left(G^{(1)}\right)_{1} \times\left(G^{(1)}\right)_{1} /\left(G^{(1)}\right)_{2}$, Phys. Lett. B 304 (1993) $263-270$.

[10] R. Kedem, T. R. Klassen, B. M. McCoy and E. Melzer, Fermionic sum representations for conformal field theory characters, Phys. Lett. B 307 (1993) 68-76.

[11] A. N. Kirillov, Bethe Ansatz and combinatorics of Young tableaux (Russian), PhD thesis, LOMI, Leningrad, 1987.

[12] A. N. Kirillov and N. Y. Reshetikhin, The Bethe Ansatz and the combinatorics of Young tableaux, J. Soviet Math. 41 (1988) 925-955.

[13] A. N. Kirillov and M. Shimozono, A generalization of the Kostka-Foulkes polynomials, preprint math.QA/9803062.

[14] D. E. Knuth, Permutations, matrices, and generalized Young tableaux, Pacific J. Math. 34 (1970) 709-727.

[15] A. Lascoux, B. Leclerc and J.-Y. Thibon, Ribbon tableaux, Hall-Littlewood functions, quantum affine algebras, and unipotent varieties, J. Math. Phys. 38 (1997) 1041-1068.

[16] A. Lascoux and M. P. Schützenberger, Sur une conjecture de H.O. Foulkes, CR Acad. Sci. Paris 286A (1978) 323-324. 
[17] A. Lascoux and M. P. Schützenberger, Le monö̈de plaxique, in Noncommutative structures in algebra and geometric combinatorics (Naples, 1978), 129-156, A. de Luca Ed., Quaderni della Ricerca Scientifica, 109, CNR, Roma, 1981.

[18] I. G. Macdonald, Symmetric functions and Hall polynomials, Oxford University Press, Oxford, second edition, 1995.

[19] J. B. Remmel and R. Whitney, Multiplying Schur functions, J. Algorithms 5 (1984) 471-487.

[20] A. Schilling and S. O. Warnaar, Inhomogeneous lattice paths, generalized Kostka polynomials and $A_{n-1}$-supernomials, to appear in Commun. Math. Phys., preprint math.QA/9802111.

[21] M. P. Schützenberger, Quelques remarques sur une construction de Schensted, Math. Scand. 12 (1963) 117-128.

[22] M. Shimozono, A cyclage poset structure for Littlewood-Richardson tableaux, preprint math.QA/9804037.

[23] M. Shimozono, Multi-atoms and monotonicity of generalized Kostka polynomials, preprint math.QA/9804038.

[24] M. Shimozono, Affine type A crystal structure on tensor products of rectangles, Demazure characters, and nilpotent varieties, preprint math.QA/9804039.

[25] M. Shimozono and J. Weyman, Graded characters of modules supported in the closure of a nilpotent conjugacy class, preprint math.QA/9804036.

[26] S. O. Warnaar, Fermionic solution of the Andrews-Baxter-Forrester model. I. Unification of TBA and CTM methods, J. Statist. Phys. 82 (1996) 657-685.

[27] S. O. Warnaar, Fermionic solution of the Andrews-Baxter-Forrester model. II. Proof of Melzer's polynomial identities, J. Statist. Phys. 84 (1996) 49-83.

[28] D. E. White, Some connections between the Littlewood-Richardson rule and the construction of Schensted, J. Combin. Theory Ser. A 30 (1981) 237-247.

Division of Mathematics, Graduate School of Science, Hokkaido UniverSITY, SAPPORO, 060-0810, JAPAN

E-mail address: kirillov@math.sci.hokudai.ac.jp

Instituut voor Theoretische Fysica, Universiteit van Amsterdam, Valckenierstraat 65, 1018 XE Amsterdam, The Netherlands

E-mail address: schillin@wins.uva.nl

Department of Mathematics, Virginia Tech, Blacksburg, VA 24061-0123, U.S.A.

E-mail address: mshimo@math.vt.edu 